\title{
Optimal Approximations by Piecewise Smooth Functions and Associated Variational Problems
}

\section{Citation}

Mumford, David Bryant, and Jayant Shah. 1989. Optimal approximations by piecewise smooth functions and associated variational problems. Communications on Pure and Applied Mathematics 42(5): 577-685.

\section{Published Version}

doi:10.1002/cpa.3160420503

\section{Permanent link}

http://nrs.harvard.edu/urn-3:HUL.InstRepos:3637121

\section{Terms of Use}

This article was downloaded from Harvard University's DASH repository, and is made available under the terms and conditions applicable to Other Posted Material, as set forth at http:// nrs.harvard.edu/urn-3:HUL.InstRepos:dash.current.terms-of-use\#LAA

\section{Share Your Story}

The Harvard community has made this article openly available.

Please share how this access benefits you. Submit a story.

\section{Accessibility}




\title{
Optimal Approximations by Piecewise Smooth Functions and Associated Variational Problems ${ }^{1}$
}

\author{
DAVID MUMFORD \\ Harvard University
}

AND

\section{JAYANT SHAH}

Northeastern University

\section{Introduction and Outline}

The purpose of this paper is to introduce and study the most basic properties of three new variational problems which are suggested by applications to computer vision. In computer vision, a fundamental problem is to appropriately decompose the domain $R$ of a function $g(x, y)$ of two variables. To explain this problem, we have to start by describing the physical situation which produces images: assume that a three-dimensional world is observed by an eye or camera from some point $P$ and that $g_{1}(\rho)$ represents the intensity of the light in this world approaching the point $P$ from a direction $\rho$. If one has a lens at $P$ focussing this light on a retina or a film-in both cases a plane domain $R$ in which we may introduce coordinates $x, y$-then let $g(x, y)$ be the strength of the light signal striking $R$ at a point with coordinates $(x, y) ; g(x, y)$ is essentially the same as $g_{1}(\rho)$-possibly after a simple transformation given by the geometry of the imaging system. The function $g(x, y)$ defined on the plane domain $R$ will be called an image. What sort of function is $g$ ? The light reflected off the surfaces $S_{i}$ of various solid objects $O_{i}$ visible from $P$ will strike the domain $R$ in various open subsets $R_{i}$. When one object $O_{1}$ is partially in front of another object $\mathrm{O}_{2}$ as seen from $P$, but some of object $\mathrm{O}_{2}$ appears as the background to the sides of $O_{1}$, then the open sets $R_{1}$ and $R_{2}$ will have a common boundary (the 'edge' of object $O_{1}$ in the image defined on $R$ ) and one usually expects the image $g(x, y)$ to be discontinuous along this boundary: see Figure 1 for an illustration of the geometry.

Other discontinuities in $g$ will be caused by discontinuities in the surface orientation of visible objects (e.g., the 'edges' of a cube), discontinuities in the objects albedo (i.e., surface markings) and discontinuities in the illumination

\footnotetext{
"A preliminary version of this paper was submitted by invitation in 1986 to "Computer Vision 1988", L. Erlbaum Press, but it has not appeared!
} 


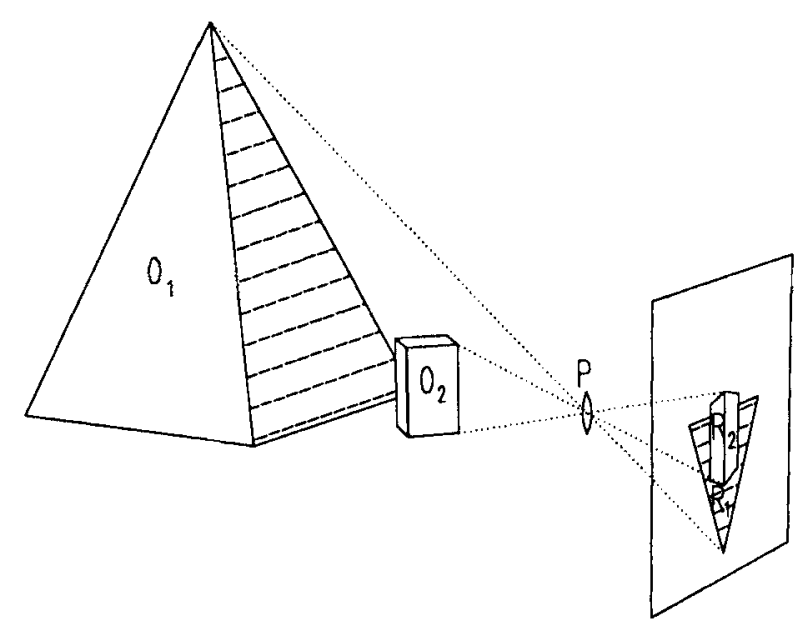

Figure 1. An image of a 3D scene.

(e.g., shadows). Including all these effects, one is led to expect that the image $g(x, y)$ is piece-wise smooth to a first approximation, i.e., it is well modelled by a set of smooth functions $f_{i}$ defined on a set of disjoint regions $R_{i}$ covering $R$. This model is, however, far from exact: (i) textured objects such as a rug or fragmented objects such as a canopy of leaves define more complicated images; (ii) shadows are not true discontinuities due to the penumbra; (iii) surface markings come in all sorts of misleading forms; (iv) partially transparent objects (e.g., liquids) and reflecting objects give further complications; (v) the measurement of $g$ always produces a corrupted, noisy approximation of the true image $g$.

In spite of all this, the piece-wise smooth model is serviceable on certain scales and to a certain approximation. Restating these ideas, the segmentation problem in computer vision consists in computing a decomposition

$$
R=R_{1} \cup \cdots \cup R_{n}
$$

of the domain of the image $g$ such that

(a) the image $g$ varies smoothly and/or slowly within each $R_{i}$,

(b) the image $g$ varies discontinuously and/or rapidly across most of the boundary $\Gamma$ between different $R_{i}$.

From the point of view of approximation theory, the segmentation problem may be restated as seeking ways to define and compute optimal approximations of a general function $g(x, y)$ by piece-wise smooth functions $f(x, y)$, i.e., functions $f$ whose restrictions $f_{i}$ to the pieces $R_{i}$ of a decomposition of the domain $R$ are differentiable. Such a problem arises in many other contexts: the perception of speech requires segmenting time, the domain of the speech signal, into intervals 
during which a single phoneme is being pronounced. Sonar, radar or laser "range" data, in which $g(x, y)$ represents the distance from a fixed point $P$ in direction $(x, y)$ to the nearest solid object, are other computer vision signals whose domains must be segmented. CAT scans are estimates of the density of the body at points $(x, y, z)$ in three-space: segmentation is needed to identify the various organs of the body.

To make mathematics out of this, we must give a precise definition of what constitutes an optimal segmentation. In this paper we shall study three functionals which measure the degree of match between an image $g(x, y)$ and a segmentation. We have a general functional $E$ which depends on two parameters $\mu$ and $\nu$ and two limiting cases $E_{0}$ and $E_{\infty}$ which depend on only one parameter $\nu$ and correspond to the limits of $E$ as the parameter $\mu$ tends to 0 and $\infty$, respectively.

We now define these three functionals and motivate them in terms of the segmentation problem. In all these functionals, we use the following notation: the $R_{i}$ will be disjoint connected open subsets of a planar domain $R$ each one with a piece-wise smooth boundary and $\Gamma$ will be the union of the part of the boundaries of the $R_{i}$ inside $R$, so that

$$
R=R_{1} \sqcup \cdots \sqcup R_{n} \sqcup \Gamma .
$$

(Conversely, we could start from a closed set $\Gamma$ made up of a finite set of singular points joined by a finite set of smooth arcs meeting only at their endpoints, and let the $R_{i}$ be the connected components of $R-\Gamma$.) For the functional $E$, let $f$ be a differentiable function on $\cup R_{i}$, which is allowed to be discontinuous across $\Gamma$. Let

$$
E(f, \Gamma)=\mu^{2} \iint_{R}(f-g)^{2} d x d y+\iint_{R-\Gamma}\|\nabla f\|^{2} d x d y+\nu|\Gamma|
$$

where $|\Gamma|$ stands for the total length of the arcs making up $\Gamma$. The smaller $E$ is, the better $(f, \Gamma)$ segments $g$ :

(i) the first term asks that $f$ approximates $g$,

(ii) the second term asks that $f$ - and hence $g$ - does not vary very much on each $R_{i}$,

(iii) the third term asks that the boundaries $\Gamma$ that accomplish this be as short as possible.

Dropping any of these three items, inf $E=0$ : without the first, take $f=0$, $\Gamma=\varnothing$; without the second, take $f=g, \Gamma=\varnothing$; without the third, take $\Gamma$ to be a fine grid of $N$ horizontal and vertical lines, $R_{i}=N^{2}$ small squares, $f=$ average of $g$ on each $R_{i}$. The presence of all three terms makes $E$ interesting.

The pair $(f, \Gamma)$ has an interesting interpretation in the context of the original computer vision problem: $(f, \Gamma)$ is simply a cartoon of the actual image $g ; f$ may be taken as a new image in which the edges are drawn sharply and precisely, 
the objects surrounded by the edges are drawn smoothly without texture. In other words, $(f, \Gamma)$ is an idealization of a true-life complicated image by the sort of image created by an artist. The fact that such cartoons are perceived correctly as representing essentially the same scene as $g$ argues that this is a simplification of the scene containing many of its essential features.

We do not know if the problem of minimizing $E$ is well posed, but we conjecture this to be true. For instance we conjecture that for all continuous functions $g, E$ has a minimum in the set of all pairs $(f, \Gamma), f$ differentiable on each $R_{i}, \Gamma$ a finite set of singular points joined by a finite set of $C^{1}$-arcs.

A closely related functional defined for functions $g$ and $f$ on a lattice instead of on a plane domain $R$ was first introduced by D. and S. Geman [6], and has been studied by Blake and Zisserman [4], J. Marroquin [10] and others. The variational problem $\delta E=0$ turns out to be related to a model introduced recently by $M$. Gurtin [8] in the study of the evolution of freezing/melting contours of a body in three-space.

The second functional $E_{0}$ is simply the restriction of $E$ to piecewise constant functions $f$ : i.e., $f=$ constant $a_{i}$ on each open set $R_{i}$. Then multiplying $E$ by $\mu^{-2}$ we have

$$
\mu^{-2} E(f, \Gamma)=\sum_{i} \iint_{R_{i}}\left(g-a_{i}\right)^{2} d x d y+\nu_{0}|\Gamma|
$$

where $\nu_{0}=\nu / \mu^{2}$. It is immediate that this is minimized in the variables $a_{i}$ by setting

$$
a_{i}=\operatorname{mean}_{R_{i}}(g)=\iint_{R_{i}} g d x d y / \operatorname{area}\left(R_{i}\right)
$$

so we are minimizing

$$
E_{0}(\Gamma)=\sum_{i} \iint_{R_{i}}\left(g-\operatorname{mean}_{R_{i}} g\right)^{2} d x d y+\nu_{0} \cdot|\Gamma|
$$

As we shall see, if $\Gamma$ is fixed and $\mu \rightarrow 0$, the $f$ which minimizes $E$ tends to a piecewise constant limit, so one can prove that $E_{0}$ is the natural limit functional of $E$ as $\mu \rightarrow 0$. $E_{0}$ may be viewed as a modification of the usual Plateau problem functional, length $(\Gamma)$, by an external force term that keeps the regions $R_{i}$-soap bubbles in the Plateau problem setting-from collapsing. Whereas the twodimensional Plateau problem has only rather uninteresting extrema with $\Gamma$ made up of straight line segments (cf. Allard-Almgren [1]), the addition of the pressure term makes the infimum more interesting. Having the powerful arsenal of geometric measure theory makes it straightforward to prove that the problem of minimizing $E_{0}$ is well posed: for any continuous $g$, there exists a $\Gamma$ made up of a finite number of singular points joined by a finite set of $C^{2}$-arcs on which $E_{0}$ 

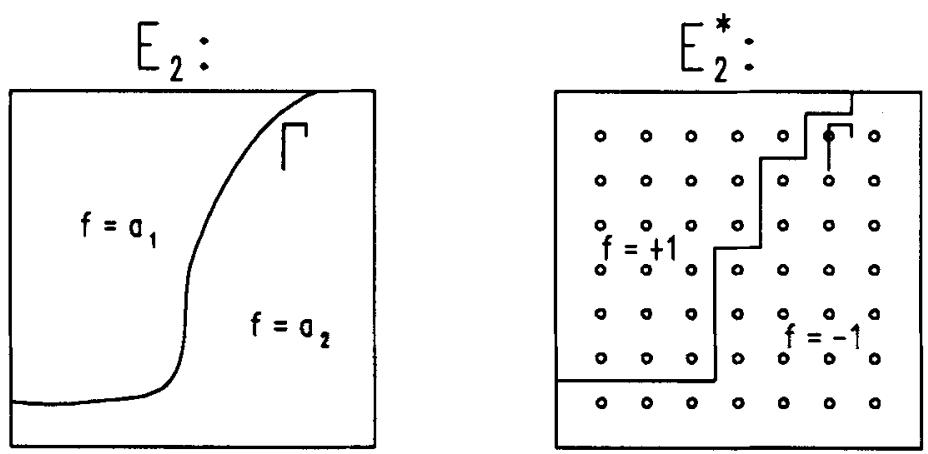

Figure 2. Continuous vs. discrete segmentation.

attains a minimum. $E_{0}$ is also closely related to the energy functional in the Ising model. For this, we restrict $f$ even further to take on only two values: +1 and -1 , and we assume $g$ and $f$ are functions on a lattice instead of functions on a two-dimensional region $R$. In this setting, $\Gamma$ is the path made up of lines between all pairs of adjacent lattice points on which $f$ changes sign (see Figure 2). Then $E_{0}$ reduces to

$$
E_{0}^{*}(f)=\sum_{\substack{\text { lattice pts } \\
i, j}}(f(i, j)-g(i, j))^{2}+\nu_{0} \cdot \sum_{\begin{array}{c}
\text { adjacent } \\
\text { lattice pts } \\
(i, j),\left(i^{\prime}, j^{\prime}\right)
\end{array}}\left(f(i, j)-f\left(i^{\prime}, j^{\prime}\right)\right)^{2}
$$

which is the Ising model energy.

The third functional $E_{\infty}$ depends only on $\Gamma$ and is given by

$$
E_{\infty}(\Gamma)=\int_{\Gamma}\left[\nu_{\infty}-\left(\frac{\partial g}{\partial n}\right)^{2}\right] d s
$$

where $\nu_{\infty}$ is a constant, $d s$ is arc length along $\Gamma$ and $\partial / \partial n$ is a unit normal to $\Gamma$. Using $d x, d y$ as coordinates on the tangent plane to $R$, so that $d s=\sqrt{d x^{2}+d y^{2}}$, $E_{\infty}$ may be rewritten as the integral along $\Gamma$ of a generalized Finsler metric $\rho(d x, d y, x, y)$ (a function $\rho$ such that $\rho(t d x, t d y, x, y)=|t| \cdot \rho(d x, d y, x, y)$ ), namely:

$$
\begin{gathered}
E_{\infty}(\Gamma)=\int_{\Gamma} \rho(d x, d y, x, y) \\
\rho(d x, d y, x, y)=\frac{\nu_{\infty}\left(d x^{2}+d y^{2}\right)-\left(g_{x} d y-g_{y} d x\right)^{2}}{\sqrt{d x^{2}+d y^{2}}} .
\end{gathered}
$$




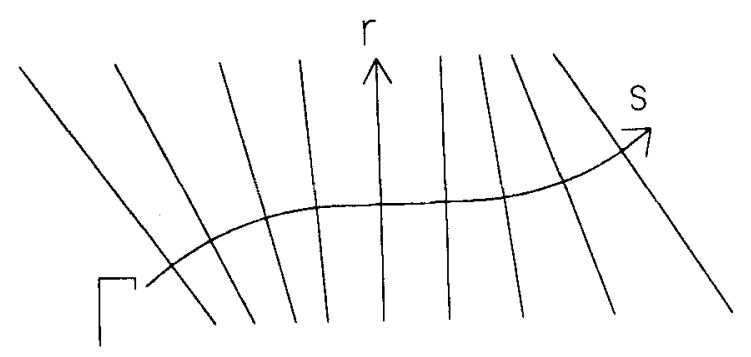

Figure 3. Curvilinear coordinates $r, s$.

Intuitively, minimizing $E_{\infty}$ is then a generalized geodesic problem. It asks for paths $\Gamma$ such that (i) length $(\Gamma)$ is as short as possible while (ii) normal to $\Gamma, g$ has the largest possible derivative. Looking at the graph of $g$ as a landscape, $\Gamma$ is the sort of path preferred by mountain goats-short but clinging to the face of cliffs wherever possible.

At first glance, $E_{\infty}$ looks completely unrelated to $E$. In fact, like $E_{0}$ it is essentially $E$ with a special choice of $f$ :

We consider only smooth parts of $\Gamma$ and take $f=g$ outside an infinitesimal neighborhood of $\Gamma$. Near $\Gamma$, set

$$
f(r, s)=g(r, s)+\varepsilon \operatorname{sgn}(r) \cdot e^{-|r| / \varepsilon} \frac{\partial g}{\partial r}(0, s)
$$

where $r, s$ are curvilinear coordinates defined by normals of $\Gamma$ (see Figure 3), and $\varepsilon$ is infinitesimal. Then if $\mu=1 / \varepsilon, \nu=2 \varepsilon \nu_{\infty}$, it can be checked that

$$
E(f, \Gamma)-E(g, \phi)=2 \varepsilon E_{\infty}(\Gamma)+O\left(\varepsilon^{2}\right)
$$

We shall see, moreover, that for fixed $\Gamma$, if $\mu$ is very large, the $f$ which minimizes $E$ is very close to the above $f$ with $\varepsilon=1 / \mu$, so this $E_{\infty}$ is a natural limit functional of $E$ as $\mu \rightarrow \infty$.

Minimizing $E_{\infty}$ over all $\Gamma$ is not a well-posed problem in most cases: if $\|\nabla g\|^{2} \leqq \nu_{\infty}$ everywhere, then $E_{\infty} \geqq 0$ and the simple choice $\Gamma=\varnothing$ minimizes $E_{\infty}$. But if $\|\nabla g\|^{2}>\nu_{\infty}$ on a non-empty open set $U$, then consider $\Gamma$ made up of many pieces of level curves of $g$ within $U$. On such $\Gamma$ 's, $E_{\infty}$ tends to $-\infty$. For $\mu$ large but not infinite, the pair $(f, \Gamma)$ which minimizes $E$ itself presumably has a $\Gamma$ made up of many components in this open set $U$. Minimizing $E_{\infty}$ on a suitably restricted class of $\Gamma$ 's can, however, be a well-posed problem.

We finish this introduction by giving an outline of the results of each section. The proofs in the later sections will sometimes be quite technical and hence it seems useful to describe the main results here before plunging into detail.

In Section 2, we analyze the variational equations for the functional $E$. Fixing $\Gamma$, standard calculus of variations shows that $E$ is a positive definite quadratic 
function in $f$ with a unique minimum. The minimum is the function $f$ which solves the elliptic boundary value problem on each $R_{i}$,

$$
\begin{gathered}
\Delta f=\mu^{2}(f-g), \\
\partial f /\left.\partial n\right|_{\partial R_{i}} \equiv 0 .
\end{gathered}
$$

Here $\partial R_{i}$ is the boundary of $R_{i}$, and $\partial / \partial n$ is a unit normal vector to $\partial R_{i}$. The second condition means that $\partial f / \partial n$ is zero on both sides of $\Gamma$ and on the inside of $\partial R$, the boundary of the whole domain $R$. Writing $f_{\Gamma}$ for this solution, $E$ reduces to a function of $\Gamma$ alone:

$$
E\left(f_{\Gamma}, \Gamma\right)
$$

Next, we make an infinitesimal variation of $\Gamma$ by a normal vector field $\mathbf{X}=a(x, y) \partial / \partial n$ defined along $\Gamma$ and zero in the neighborhood of the singular points of $\Gamma$. We prove:

$$
\frac{\delta}{\delta X} E\left(f_{\Gamma}, \Gamma\right)=\int_{\Gamma} a\left(e_{+}-e_{-}+\nu \cdot \operatorname{curv}(\Gamma)\right) d s,
$$

where

$$
\begin{aligned}
& e_{ \pm}=\mu^{2}\left(f_{\Gamma}^{ \pm}-g\right)^{2}+\left(\frac{d f_{\Gamma}^{ \pm}}{d s}\right)^{2}, \\
& f_{\Gamma}^{ \pm}=\text {boundary values of } f_{\Gamma} .
\end{aligned}
$$

Therefore if $E\left(f_{\Gamma}, \Gamma\right)$ is minimized at $\Gamma, \Gamma$ must satisfy the variational equation

$$
e_{+}-e_{-}+\nu \cdot \operatorname{curv}(\Gamma) \equiv 0
$$

Finally, we look at possible singular points of $\Gamma$. Here the situation is complicated by the fact that although the minimizing $f_{\Gamma}$ is pointwise bounded:

$$
\min _{R} g \leqq f_{\Gamma}(P) \leqq \max _{R} g,
$$

its gradient may be unbounded in a neighborhood of the singular points of $\Gamma$. However, the behavior of the solutions of elliptic boundary value problems in domains with "corners" has been studied by several people, starting with a classic paper of Kondratiev [9] and recently surveyed in a book of Grisvard [7]. Using these bounds, and assuming that the singular points are given by a finite number of $C^{2}$-arcs with a common endpoint, it is easy to show, by elementary comparisons of $E\left(f_{\Gamma}, \Gamma\right)$ with $E$ on modified $\Gamma$ 's, that if $E\left(f_{\Gamma}, \Gamma\right)$ attains a minimum at 
some $\Gamma$, then the only possible singularities of $\Gamma$ are:

(i) “triple points" $P$ where three $C^{2}$-arcs meet with $120^{\circ}$-angles,

(ii) "crack-tips" $P$ where a single $C^{2}$-arc ends and no other arc meets $P$.

Moreover, on the boundary of the domain $R$, another possibility is:

(iii) "boundary points" $P$ where a single $C^{2}$-arc of $\Gamma$ meets perpendicularly a smooth point of $\partial R$.

There is a wrinkle here though: assuming that $E$ has a minimum when $\Gamma$ varies over some reasonable set of possible curves, it is not clear that the minimizing $\Gamma$ will have singular points made up of $C^{2}$-arcs. Instead there might well be a larger class of nastier singular points that can arise as minima. In Section 3, we look more closely at crack-tips with this possibility in mind. First of all, assuming the crack-tip is $C^{2}$ we calculate the first variation of $E$ with respect to infinitesimal extensions or truncations of $\Gamma$ at the crack tip. We find a new restriction on minimizing $\Gamma$ 's, which is the analogue of Griffiths' law of cracks in solid mechanics. Secondly, we consider the possibility that the crack-tip might be given by

$$
y=\alpha x^{3 / 2}+\phi(x), \quad x \geqq 0, \phi \text { a } C^{2} \text {-function. }
$$

Such singular points are called cusps in algebraic geometry. We give several arguments to make it plausible that such a singularity will occur on minimizing $\Gamma$ 's. Moreover, in the natural world, approximate cusps certainly look like they appear at the ends of arcs in other situations which may be modelled by free boundary value problems. For example, consider sand bars that stick out into an area with a strong transverse current. Sand may be washed away or may accrete, hence the boundary is free to shift in both directions, and its tip may likewise erode or grow. Figure 4 shows a chart of Cape Cod and of Monomoy Island, whose outlines are strongly reminiscent of cusps.

In Section 4, we study $E$ for small $\mu$. We use the isoperimetric constant $\lambda_{\Gamma}$ which gets small only if some component $W$ of $R-\Gamma$ has a narrow "neck" (see Figure 5). By definition, $\lambda_{\Gamma}$ is the minimum of $\delta / \min \left(A_{1}, A_{2}\right)$ over all diagrams as in the figure. We prove that

$$
\mu^{2}\left\{E_{0}(\Gamma)-\frac{4 \mu^{2}}{\lambda_{\Gamma}^{2}+4 \mu^{2}} \iint g^{2}\right\} \leqq E\left(f_{\Gamma}, \Gamma\right) \leqq \mu^{2} E_{0}(\Gamma)
$$

where $\nu_{0}=\nu / \mu^{2}$. We then study the first variation of $E_{0}(\Gamma)$. We show firstly that the first variation of $E\left(f_{\Gamma}, \Gamma\right)$ also tends to the first variation of $E_{0}(\Gamma)$. Secondly, the equation for the first variation of $E_{0}(\Gamma)$ being zero turns out to be the second-order differential equation for $\Gamma$ :

$$
\left(\bar{g}_{\Gamma}^{+}-\bar{g}_{\Gamma}^{-}\right) \cdot\left(\bar{g}_{\Gamma}^{+}+\bar{g}_{\Gamma}^{-}-2 g\right)+\nu_{0} \cdot \operatorname{curv}(\Gamma) \equiv 0,
$$




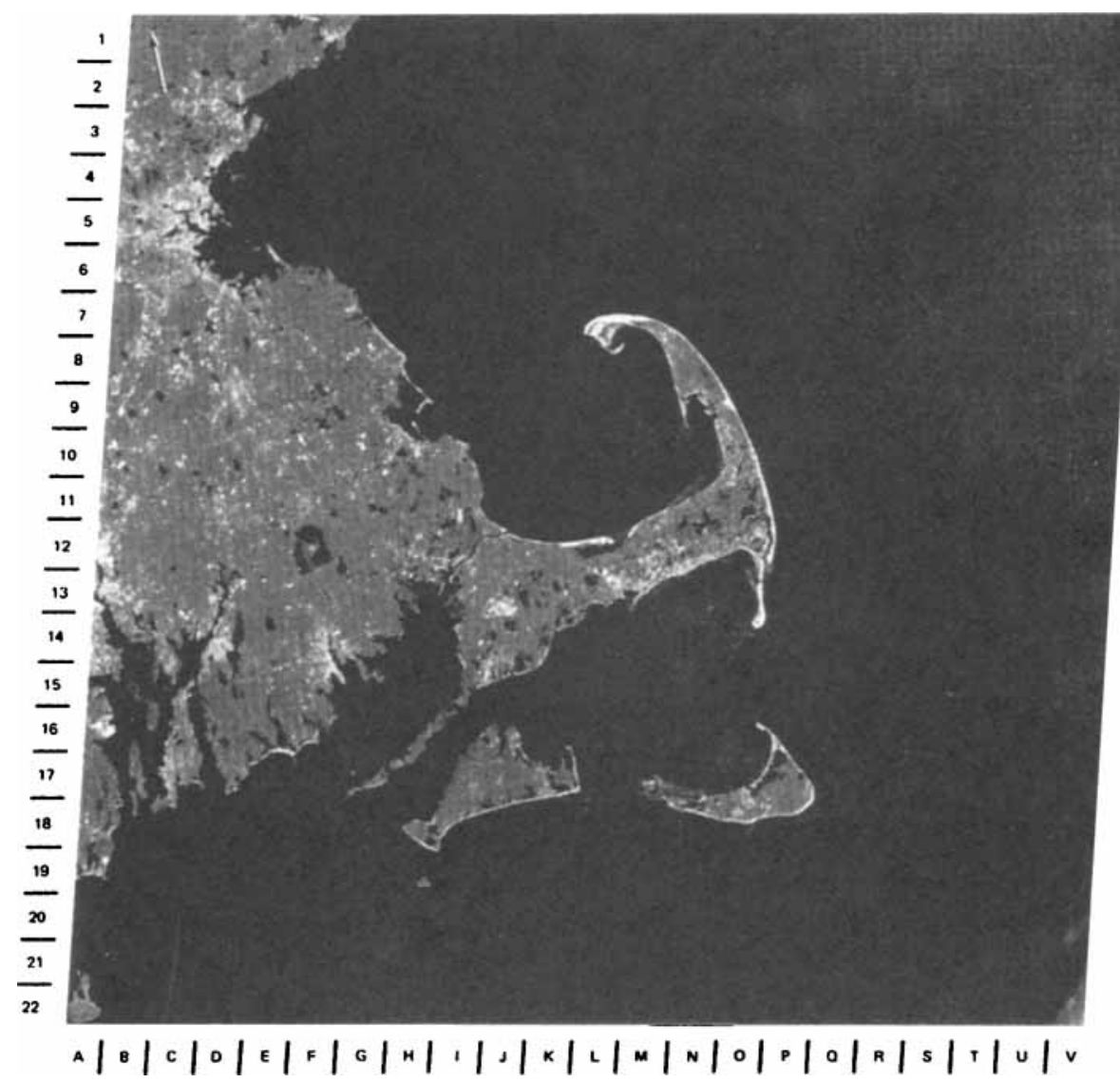

Figure 4. A sandy shore of ten has cusp-like spits.

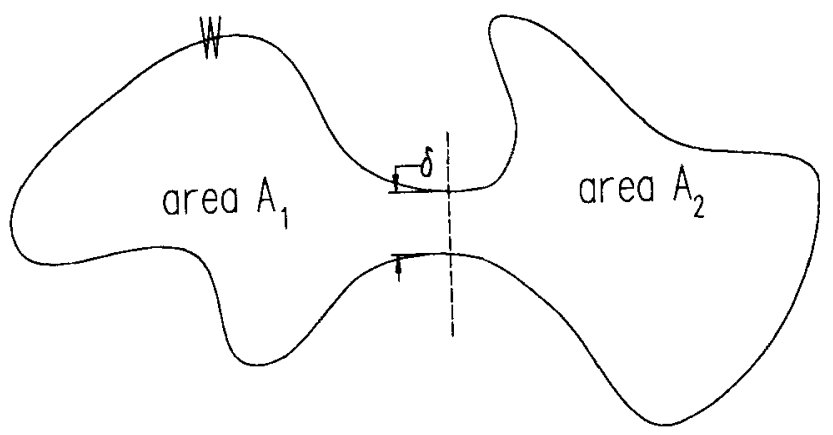

Figure 5. Definition of the isoperimetric constant. 
where $\bar{g}_{\Gamma}^{+}$and $\bar{g}_{\Gamma}$ are the means of $g$ on the components of $R-\Gamma$ to each side of $\Gamma$.

Section 5 is devoted to the proof that $\min E_{0}(\Gamma)$ exists for a $\Gamma$ which is a finite union of $C^{2}$-arcs joining a finite number of triple points or border points. We dip deeply into the toolkit of geometric measure theory, using especially the results in Simon [13]. The fundamental idea is to enlarge the set of allowable $\Gamma$ 's until one of the compactness results of geometric measure theory can be applied to show that $\min E_{0}$ exists for a possibly very wild $\Gamma$. And once this $\Gamma$ is in hand, use the vanishing of its first variation to show that $\Gamma$ is in fact very nice. More precisely, what we do is to shift the focus from $\Gamma$ to a decomposition of $R$ into disjoint measurable sets:

$$
R=R_{1} \sqcup \cdots \sqcup R_{n}
$$

and define

$$
E_{n}\left(\left\{R_{i}\right\}\right)=\sum_{i}\left(\iint\left(g-\operatorname{mean}_{R_{i}} g\right)^{2} d x d y+\frac{1}{2} \nu_{0}\left|\partial R_{i}\right|\right)
$$

For this to make sense, $R_{i}$ must be a so-called Cacciopoli set, a measurable set whose boundary as a current $\partial R_{i}$ has finite length, $\left|\partial R_{i}\right|$. Then, for each $n$, we show that $E_{n}$ attains its minimum, and that at this minimum, $R_{i}$ is an open set (up to a measure zero set) with piecewise $C^{2}$ boundary. We show finally that as $n$ increases, the minimum eventually increases if all $R_{i}$ have positive measure. How should Cacciopoli sets be visualized? Note that the boundaries of Cacciopoli sets are not fractals: their Hausdorff dimension is 1 since their length is finite, but they will, in general, be made up of infinitely many rectifiable arcs with finite total length. A good way to imagine what Cacciopoli sets are like is to look at the segmentation of the world into sea and land. Some coastlines are best seen as fractals: Richardson's data on the indefinite growth of the length of rocky coasts with the scale of the yardstick was one of the inspirations of Mandelbrot's theory of fractals. But other coasts are mixtures of smooth and ragged parts, and may serve as models (see Figure 6).

In Section 6 we study $E$ for large $\mu$. The first step is to use Green's theorem and rewrite $E$ as an integral only over $\Gamma$ : let $g_{\mu}$ and $f_{\Gamma}$ be the solutions of

$$
\begin{array}{ll}
\Delta g_{\mu}=\mu^{2}\left(g_{\mu}-g\right), & \Delta f_{\Gamma}=\mu^{2}\left(f_{\Gamma}-g\right), \\
\partial g_{\mu} /\left.\partial n\right|_{\partial R} \equiv 0, & \partial f_{\Gamma} /\left.\partial n\right|_{(\Gamma \cup \partial R)} \equiv 0 .
\end{array}
$$

Then we show that

$$
E\left(f_{\Gamma}, \Gamma\right)=E\left(g_{\mu}, \phi\right)+\int_{\Gamma}\left[\nu-\frac{\partial g_{\mu}}{\partial n}\left(f_{\Gamma}^{+}-f_{\Gamma}^{-}\right)\right] d s
$$




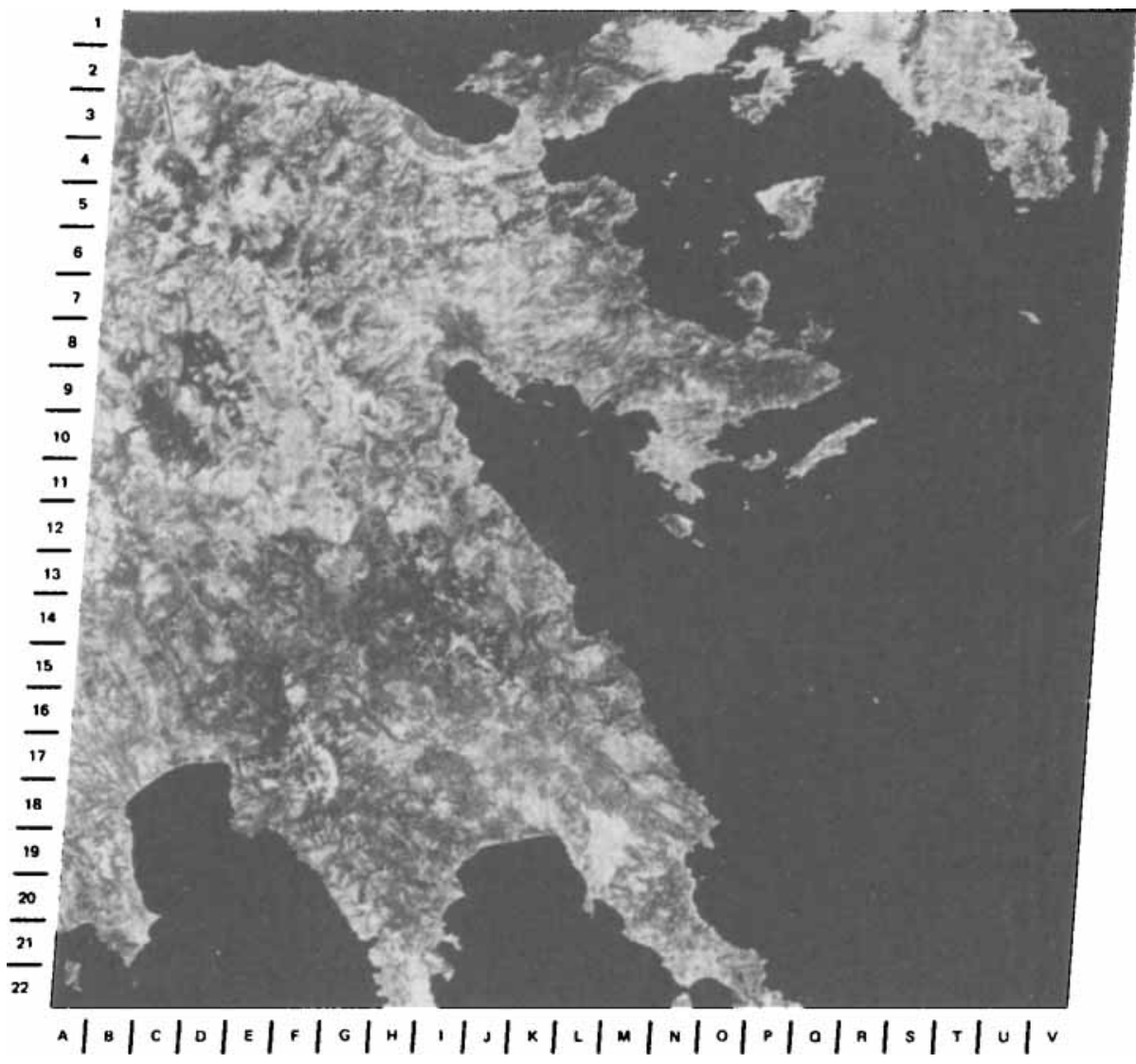

Figure 6. A Cacciopoli-like coastline.

where $f_{\Gamma}^{ \pm}$are the boundary values of $f_{\Gamma}$ along $\Gamma$ and $\partial / \partial n$ points from the side of $\Gamma$ to the + side. Moreover, if $1 / \mu$ is small, we prove that, away from the singularities of $\Gamma$,

$$
f_{\Gamma}^{+}-f_{\Gamma}^{-}=\frac{2}{\mu} \frac{\partial g_{\mu}}{\partial n}+O\left(\frac{1}{\mu^{2}}\right),
$$

while uniformly on $R$

$$
f_{\Gamma}-g=o\left(\frac{1}{\mu}\right)
$$

from which we deduce that

$$
E\left(f_{\Gamma}, \Gamma\right)=E\left(g_{\mu}, \phi\right)+\frac{2}{\mu} E_{\infty}(\Gamma)+O\left(\frac{\log \mu}{\mu^{2}}\right),
$$


where $\nu_{\infty}=\frac{1}{2} \mu \nu$. We then study the first variation of $E_{\infty}(\Gamma)$. We show firstly that the variation of $E\left(f_{\Gamma}, \Gamma\right)$ also tends to the first variation of $E_{\infty}(\Gamma)$. Secondly, the equation for the first variation of $E_{\infty}(\Gamma)$ being zero turns out to be a second-order differential equation for $\Gamma$. Let $H_{g}$ be the matrix of second derivatives of $g$, let $t_{\Gamma}$ and $n_{\Gamma}$ be the unit tangent vector and unit normal vector for $\Gamma$. Then the equation is

$$
\begin{aligned}
& \left(n_{\Gamma} \cdot \nabla g\right) \cdot \Delta g+\left(t_{\Gamma} \cdot \nabla g\right) \cdot\left(t_{\Gamma} \cdot H_{g} \cdot n_{\Gamma}\right) \\
& \quad+\operatorname{curv}(\Gamma) \cdot\left[\frac{1}{2} \nu_{\infty}+\frac{1}{2}\left(n_{\Gamma} \cdot \nabla g\right)^{2}-\left(t_{\Gamma} \cdot \nabla g\right)^{2}\right]=0 .
\end{aligned}
$$

(To read this equation properly, note that $\nabla g, \Delta g$ and $H_{g}$ play the role of coefficients, $t_{\Gamma}$ and $n_{\Gamma}$ are 1-st derivatives of the solution curve and $\operatorname{curv}(\Gamma)$ is its 2-nd derivative.)

Finally, in Section 7, we look briefly at this equation, noting that like the equation for geodesics in a Lorentz metric, it has two types of local solutions: "space-like" solutions which locally minimize $E_{\infty}$ and "time-like" solutions which locally maximize $E_{\infty}$. A general solution flips back and forth between the two types, with cusps marking the transition. Discussion of existence theorems for solutions of this differential equation is postponed to a later paper.

We use the following standard notations throughout this paper:

(a) For $k \geqq 0$ an integer and $1 \leqq p \leqq \infty, W_{p}^{k}(D)$ is the Banach space of functions $f$ on $D$ with norm

$$
\|f\|_{k, p, D}=\left[\sum_{\substack{\alpha, \beta \geqq 0 \\ \alpha+\beta \leqq k}} \iint_{D}\left|\frac{\partial^{2} f}{\partial x^{\alpha} \partial y^{\beta}}\right|^{p} d x d y\right]^{1 / p}
$$

(b) $C^{k}$ is the class of functions with continuous derivatives through order $k$, and $C^{k, 1}$ of those whose $k$-th derivatives satisfy a Lipshitz condition. The boundary of a domain $D$ is said to be in one of these classes if $D$ is represented locally by $y<f(x)$ (or $y>f(x)$ or $x<f(y)$ or $x>f(y)$ ), where $f$ belongs to the class.

\section{The First Variation}

As described in Section 1, we fix a region $R$ in the plane with compact closure and piecewise smooth boundary and we fix a continuous function $g$ on $\bar{R}$. We also fix positive constants $\mu$ and $\nu$. We consider next a subset $\Gamma \subset R$ made up of a finite set $\left\{\gamma_{\alpha}\right\}$ of curves. We shall assume for our analysis initially that the $\gamma_{\alpha}$ are simple $C^{1,1}$-curves meeting $\partial R$ and meeting each other only at their endpoints. Finally, we consider a function $f$ on $R-\Gamma$ which we take initially to be 
$C^{1}$ with first derivatives continuous up to all boundary points. ${ }^{2}$ For each $f$ and $\Gamma$, we have the functional $E$ defined in Section 1:

$$
E(f, \Gamma)=\mu^{2} \iint_{R}(f-g)^{2} d x d y+\iint_{R-\Gamma}\|\nabla f\|^{2} d x d y+\nu|\Gamma|
$$

The fundamental problem is to find $f$ and $\Gamma$ which minimize the value of $E$. Note that by a scaling in the coordinates $x, y$ in $R$ and by a multiplicative constant in thë functions $f, g$, we can transform $E$ with any constants $\mu, \nu$ into the $E$ for any other set of constants $\mu, \nu$. Put another way, if $\mu$ is measured in units of inverse distance in the $R$ - plane, and the constant $\nu$ is measured in units of (size of $g)^{2} /$ distance, then the three terms of $E$ all have the same "dimension". So fixing $\mu, \nu$ is the same as fixing units of distance in $R$ and of size of $g$. The purpose of this section is to study the effect of small variations of $f$ and $\Gamma$ on $E$ and determine the condition for the first variation of $E$ to be 0 . These are, of course, necessary conditions for $f$ and $\Gamma$ to minimize $E$.

The first step is to fix $\Gamma$ as well as $g$ and vary $f$. This is a standard variational problem. Let $\delta f$ represent a function of the same type as $f$. Then

$$
\begin{aligned}
& E(f+t \delta f, \Gamma)-E(f, \Gamma) \\
& =t\left[\mu^{2} \iint 2 \delta f \cdot(f-g) d x d y+\iint 2(\nabla(\delta f) \cdot \nabla f) d x d y\right] \\
& \quad+t^{2}\left[\mu^{2} \iint(\delta f)^{2} d x d y+\iint\|\nabla(\delta f)\|^{2} d x d y\right] .
\end{aligned}
$$

Thus

$$
\begin{aligned}
\frac{\delta E}{\delta f}(f, \Gamma) & =\lim _{t \rightarrow 0} \frac{E(f+t \delta f, \Gamma)-E(f, \Gamma)}{t} \\
& =2\left[\mu^{2} \iint \delta f \cdot(f-g) d x d y+\iint(\nabla(\delta f) \cdot \nabla f) d x d y\right] .
\end{aligned}
$$

Integrating by parts and using Green's theorem ${ }^{3}$ we obtain

$$
\begin{aligned}
\frac{1}{2} \frac{\delta E}{\delta f}(f, \Gamma) & =\mu^{2} \iint \delta f \cdot(f-g) d x d y-\iint \delta f \cdot \nabla^{2} f d x d y+\int_{B} \delta f \frac{\partial f}{\partial n} d s \\
& =\iint \delta f\left(\nabla^{2} f-\mu^{2}(f-g)\right) d x d y+\int_{B} \delta f \frac{\partial f}{\partial n} d s
\end{aligned}
$$

\footnotetext{
${ }^{2}$ We shall weaken these conditions later to include certain $f$ 's with more complicated boundary behavior, but this will not affect the initial arguments. Appendix 1 contains a full discussion of how regular the minimizing functions $f$ will be at different points of $R$.

${ }^{3}$ For Green's theorem to be applicable, we must assume that $f \in W_{p}^{2}$, some $p \geqq 1$. Alternatively, by the last equation, a minimum $f$ is a weak solution of $\nabla^{2} f=\mu^{2}(f-g)$ and, by the results in Appendix $1, f$ in fact has $L^{p}$ second derivatives locally everywhere in $R-\Gamma$.
} 
where $B$ is the entire boundary of $R-\Gamma$, i.e., $\partial R$ and each side of $\Gamma$. Now taking $\delta f$ to be a "test function", non-zero near one point of $R-\Gamma$, zero elsewhere, and taking the limit over such $\delta f$, we deduce as usual that $f$ satisfies

$$
\nabla^{2} f=\mu^{2}(f-g)
$$

on $R-\Gamma$. Now taking $\delta f$ to be non-zero near one point of $B$, zero elsewhere, we also see that

$$
(* *) \quad \frac{\partial f}{\partial n}=0 \text { on } \partial R, \text { and on the two sides } \gamma_{\alpha}^{ \pm} \text {of each } \gamma_{\alpha} .
$$

As is well known, this determines $f$ uniquely: the operator $\mu^{2}-\nabla^{2}$ is a positive-definite selfadjoint operator, and there is a Green's function $K(x, y ; u, v)$ which is $C^{\infty}$ except on the diagonal $(x, y)=(u, v)$ where it has a singularity like

$$
-\frac{1}{2 \pi} \log \left(\mu \sqrt{(x-u)^{2}+(y-v)^{2}}\right)
$$

such that, for each component $R_{i}$ of $R-\Gamma$, and for each $g$,

$$
f(x, y)=\mu^{2} \cdot \iint_{(u, v) \in R_{i}} K(x, y ; u, v) g(u, v) d u d v
$$

satisfies (*) and (**) on $R_{i}$. Further regularity results for this boundary value problem especially on the behavior of $f$ near $\Gamma$ are sketched in Appendix 1 and will be quoted as needed.

If there were no boundaries at all, then Green's function for this operator and the whole plane is obtained from the so-called "modified Bessel's function of the second kind" $K_{0}$ (cf. Whittaker and Watson [14], Section 17.71, pp. 373-374):

$$
K(x, y ; u, v)=\frac{1}{2 \pi} K_{0}\left(\mu \sqrt{(x-a)^{2}+(y-v)^{2}}\right),
$$

where $K_{0}(r)$ (defined for $r>0$ ) is the solution of

$$
K_{0}^{\prime \prime}+\frac{1}{r} K_{0}^{\prime}-K_{0}=0
$$

such that

$$
\begin{aligned}
& K_{0}(r) \sim \log (1 / r) \quad \text { for } \quad r \text { small, } \\
& K_{0}(r) \sim \sqrt{\pi / 2 r} e^{-r} \text { for } r \text { large. }
\end{aligned}
$$

Its graph is depicted in Figure 7. 


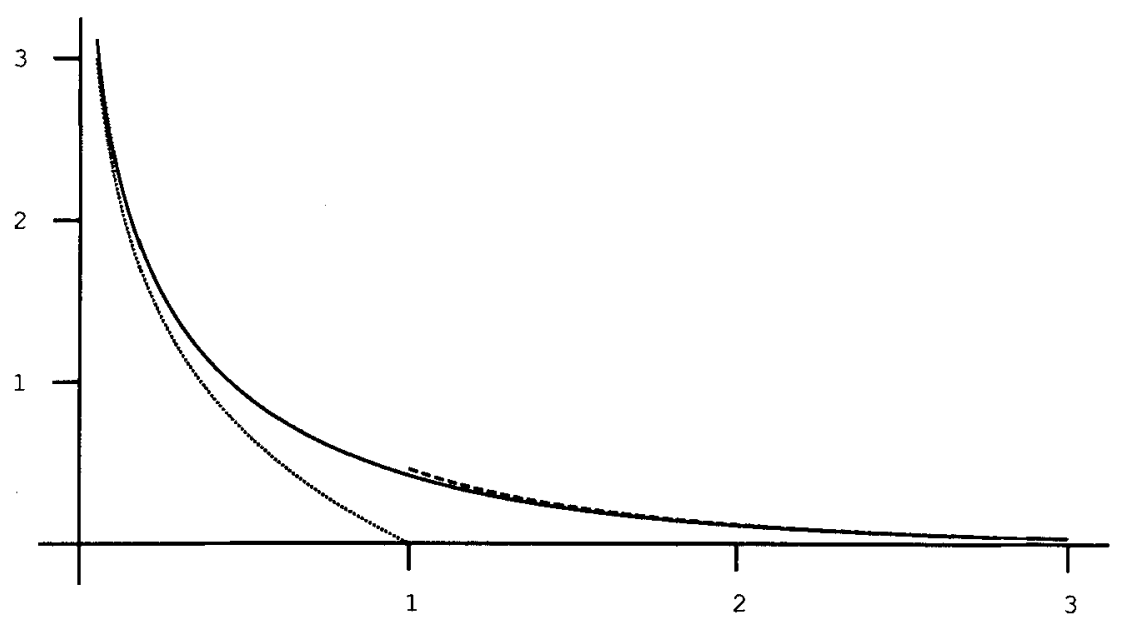

Figure 7. Graph of the Green's function and its asymptotes.

Another way of characterizing $K$ is that it is the Fourier transform of

$$
L(\xi, \eta)=1 /\left(\mu^{2}+\xi^{2}+\eta^{2}\right)
$$

evaluated at $(x-u, y-v)$.

Now look at the variation of $E$ with respect to $\Gamma$. First consider moving $\Gamma$ near a simple point $P$ of $\Gamma$. Such a $P$ lies on exactly one $\gamma_{\alpha}$ and, as $\gamma_{\alpha}$ is $C^{1,1}$, we can write it in a small neighborhood $U$ of $P$ either as a graph $y=h(x)$, or as a graph $x=h(y)$. Assume the former; then we can deform $\gamma_{\alpha}$ to

$$
y=h(x)+t \cdot \delta h(x)
$$

where $\delta h$ is zero outside a small neighborhood of $x(P)$ (see Figure 8). If $t$ is small, the new curve $\gamma_{\alpha}(t)$ does not meet any other $\gamma_{\beta}$ 's except at its endpoints and

$$
\Gamma(t)=\gamma_{\alpha}(t) \cup \bigcup_{\beta \neq \alpha} \gamma_{\beta}
$$

is an allowable deformation of $\Gamma$. Now we cannot speak of leaving $f$ fixed while $\Gamma$ moves, because $f$ must be $C^{1}$ on $R-\Gamma$ but will usually be discontinuous across $\Gamma$. Instead do this: Let $f^{+}$denote the function $f$ in

$$
U^{+}=\{(x, y) \mid y>h(x)\} \cap U
$$

and let $f^{-}$denote $f$ in

$$
U^{-}=\{(x, y) \mid y<h(x)\} \cap U
$$




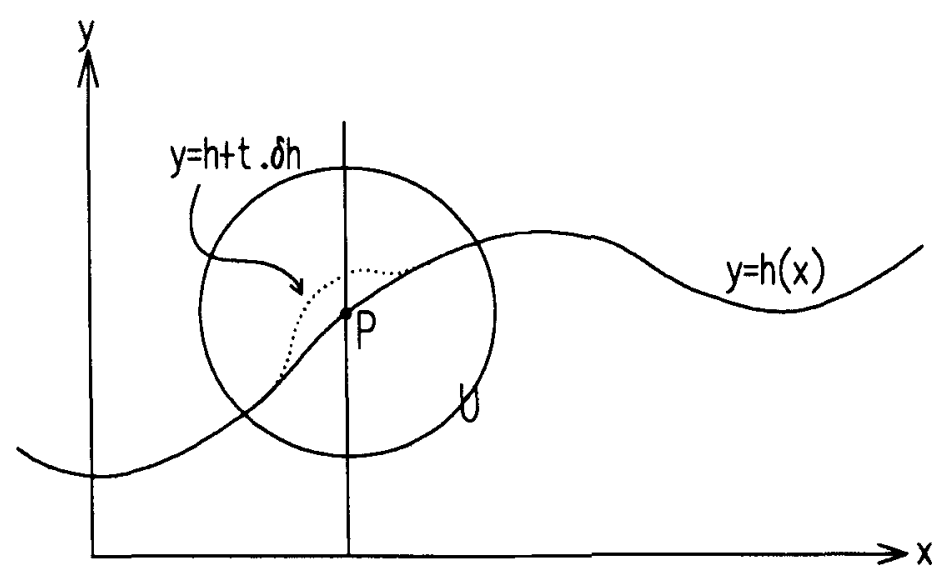

Figure 8. Deformation of $\Gamma$.

and choose $C^{1}$ extensions of $f^{+}$from $U^{+}$to $U$ and of $f^{-}$from $U^{-}$to $U$. By the results in Appendix 1, $f$ is $C^{1}$ on both sides of $\Gamma$ at all simple points of $\Gamma$, hence this is possible. Then for any small $t$, define

$$
f^{t}(P)= \begin{cases}f(P) & \text { if } P \notin U, \\ \text { extension of } f^{+} & \text {if } P \in U, P \text { above } \gamma_{\alpha}(t) \\ \text { extension of } f^{-} & \text {if } P \in U, P \text { below } \gamma_{\alpha}(t)\end{cases}
$$

Now

$$
\begin{aligned}
E\left(f^{t}, \Gamma(t)\right)-E(f, \Gamma)= & \mu^{2} \iint_{U}\left[\left(f^{t}-g\right)^{2}-(f-g)^{2}\right] d x d y \\
& +\iint_{U-\Gamma(t)}\left\|\nabla f^{t}\right\|^{2} d x d y-\iint_{U-\Gamma}\|\nabla f\|^{2} d x d y \\
& +\nu\left[\left|\gamma_{\alpha}(t)\right|-\left|\gamma_{a}\right|\right] \\
= & \mu^{2} \int\left(\int_{h(x)}^{h(x)+t \delta h(x)}\left[\left(f^{-}-g\right)^{2}-\left(f^{+}-g\right)^{2}\right] d y\right) d x \\
& +\int\left(\int_{h(x)}^{h(x)+t \delta h(x)}\left[\left\|\nabla f^{-}\right\|^{2}-\left\|\nabla f^{+}\right\|^{2}\right] d y\right) d x \\
& +\nu \int\left[\sqrt{1+(h+t \delta h)^{\prime 2}}-\sqrt{1+h^{\prime 2}}\right] d x
\end{aligned}
$$


hence

$$
\begin{aligned}
\frac{\delta E}{\delta \gamma}= & \left.\mu^{2} \int\left[\left(f^{-}-g\right)^{2}-\left(f^{+}-g\right)^{2}\right]\right|_{y=h(x)} \delta h d x \\
& +\left.\int\left[\left\|\nabla f^{-}\right\|^{2}-\left\|\nabla f^{+}\right\|^{2}\right]\right|_{y=h(x)} \delta h d x \\
& +\nu \int \frac{h^{\prime}}{\sqrt{1+h^{\prime 2}}}(\delta h)^{\prime} d x .
\end{aligned}
$$

Since $\gamma_{\alpha}$ is in $C^{1,1}$, it has a curvature almost everywhere which is bounded and is given by

$$
\operatorname{curv}\left(\gamma_{\alpha}\right) \text { at }(x, h(x))=\frac{h^{\prime \prime}(x)}{\left(1+h^{\prime}(x)^{2}\right)^{3 / 2}}
$$

where curv $>0$ means that $\gamma_{\alpha}$ is convex upwards (i.e., $U_{+}$is convex, $U_{-}$is not) while curv $<0$ means $\gamma_{\alpha}$ is convex downwards (i.e., $U_{-}$is convex, $U_{+}$is not). Integrating by parts, we get

$$
\begin{gathered}
\frac{\delta E}{\delta \gamma}=\int_{\gamma_{\alpha}}\left[\left(\mu^{2}\left(f^{-}-g\right)^{2}+\left\|\nabla f^{-}\right\|^{2}\right)-\left(\mu^{2}\left(f^{+}-g\right)^{2}+\left\|\nabla f^{+}\right\|^{2}\right)\right] \cdot \frac{\delta h}{\sqrt{1+h^{\prime 2}}} d s \\
-\nu \operatorname{curv}\left(\gamma_{\alpha}\right)
\end{gathered}
$$

( $d s=$ infinitesimal arc length on $\gamma_{\alpha}$ ). Note that the coefficient $\delta h / \sqrt{1+h^{\prime 2}}$ has the simple interpretation as the amount of the displacement of the deformed $\Gamma$ along the normal lines to the original $\Gamma$. Since this formula for $\delta E / \delta \gamma$ holds for any $\delta h$, at an extremum of $E$ we deduce that, along each $\gamma_{\alpha}$,

$$
\begin{gathered}
(* * *) \quad\left[\mu^{2}\left(f^{+}-g\right)^{2}+\right. \\
\left.\left\|\nabla f^{+}\right\|^{2}\right]-\left[\mu^{2}\left(f^{-}-g\right)^{2}+\left\|\nabla f^{-}\right\|^{2}\right] \\
+\nu \cdot \operatorname{curv}\left(\gamma_{\alpha}\right)=0 .
\end{gathered}
$$

The terms in square brackets can be simply interpreted as the energy density corresponding to the functional $E$ just above and just below the curve. In fact, write the energy density as $e$ :

$$
e(f ; x, y)=\mu^{2}(f(x, y)-g(x, y))^{2}+\|\nabla f(x, y)\|^{2}
$$

then $(* * *)$ says

$$
\left(* * *^{\prime}\right) \quad e\left(f^{+}\right)-e\left(f^{-}\right)+\nu \cdot \operatorname{curv}\left(\gamma_{\alpha}\right)=0 \text { on } \gamma_{\alpha} .
$$


(Note that if we fix $f^{+}$and $f^{-}$, then what we have here is a 2-nd order ordinary differential equation for $\gamma_{\alpha}$ )

Next, we look at special points $P$ of $\Gamma$ :

(a) points $P$ where $\Gamma$ meets the boundary of the region $R$,

(b) "corners" $P$ where two $\gamma_{\alpha}$ 's meet,

(c) "vertices" $P$ where three or more $\gamma_{\alpha}$ 's meet,

(d) "crack-tips" $P$ where a $\gamma_{\alpha}$ ends but does not meet any other $\gamma_{\beta}$ or $\partial R$.

Before analyzing the restrictions at such points implied by the stationarity of $E$, we should reconsider what assumptions may reasonably be placed on $f$. We saw above that for each fixed $\Gamma$, there was a unique minimizing $f$ : the $f$ satisfying the elliptic boundary value problem $(*),(* *)$. If $\Gamma$ is $C^{1,1}$ and $g$ is continuous, then the standard theory of elliptic operators sketched in Appendix 1 implies that $f$ is $C^{1}$ on the open set $R-\Gamma$ and that, at all simple boundary points of $\Gamma$ and $\partial R$, $f$ extends locally to a $C^{1}$-function on the region plus its boundary. This shows that $(* * *)$ certainly holds along each $\gamma_{\alpha}$. However, at corners, $p$ is not always such a nice function. In fact, the typical behavior of $f$ at a corner point with angle $\alpha$ is shown in the following example:

Let $z, w$ be complex variables and consider the conformal map $w=z^{\pi / \alpha}$ between $R_{1}$ and $R_{2}$ (see Figure 9). Let $z=r e^{i \theta}$ and let

$$
\begin{aligned}
f(z) & =\mathscr{I}_{m}(w) \\
& =r^{\pi / \alpha} \sin \left(\frac{\pi}{\alpha} \theta\right) .
\end{aligned}
$$

Then $f$ is a real-valued function on $R_{1}$ with

$$
\begin{aligned}
& \nabla^{2} f=0 \\
& \frac{\partial f}{\partial n}=0 \quad \text { on } \quad \partial R_{1} .
\end{aligned}
$$

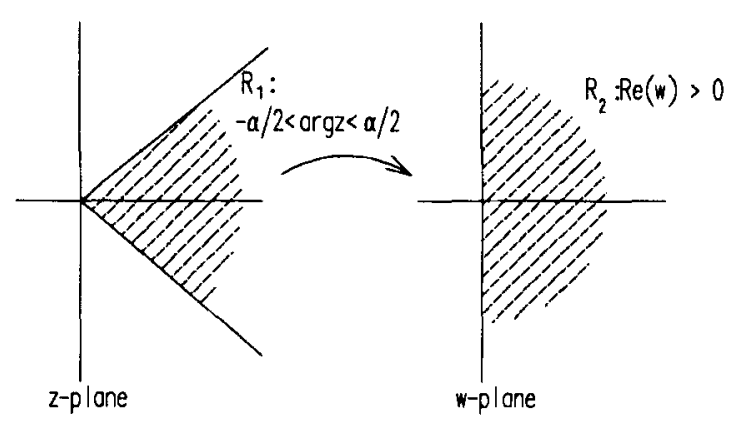

Figure 9. Straightening of a corner by a conformal map. 
But note that

$$
\frac{\partial f}{\partial r}=\frac{\pi}{\alpha} r^{\pi / \alpha-1} \sin \left(\frac{\pi}{\alpha} \theta\right)
$$

has an infinite limit as $r \rightarrow 0$ if $\alpha>\pi$.

V. A. Kondratiev [9] discovered that this type of singularity characterizes what happens to the solutions of elliptic boundary value problems at corners or even in "conical singular points" of $n$-dimensional domains. We have collected the results we shall use in Appendix 1, where it is shown that

(a) $f$ is bounded everywhere,

(b) $f$ is $C^{1}$ at corners $P$ of $R-\Gamma$ whose angle $\alpha$ satisfies $0<\alpha<\pi$,

(c) at corners $P$ such that $\pi<\alpha \leqq 2 \pi$, including the exterior of crack (i.e., $P$ is the endpoint of a $C^{1,1}$-arc which is not continued by any other arc), $f$ has the form

$$
f=c r^{\pi / \alpha} \sin \left(\frac{\pi}{\alpha}\left(\theta-\theta_{0}\right)\right)+\tilde{f}
$$

where $\tilde{f}$ is $C^{1},(r, \theta)$ are polar coordinates centered at $P$ and $c, \theta_{0}$ are suitable constants.

Using these estimates, let us first show that if $E(f, \Gamma)$ is minimum, then $\Gamma$ has no kinks, i.e., points $P$ where two edges $\gamma_{i}$ and $\gamma_{j}$ meet at an angle other than $\pi$. To do this, let $U$ be a disc around $P$ of radius $\varepsilon$ and let $\gamma_{i} \cup \gamma_{j}$ divide $U$ into sectors $U^{-}$with angle $\alpha^{-}>\pi$ and $U^{+}$with angle $\alpha^{+}<\pi$ (see Figure 10). We assume $0<\alpha^{+}<\pi$ and hence $\pi<\alpha^{-}<2 \pi$. Fix a $C^{\infty}$-function $\eta(x, y)$ such

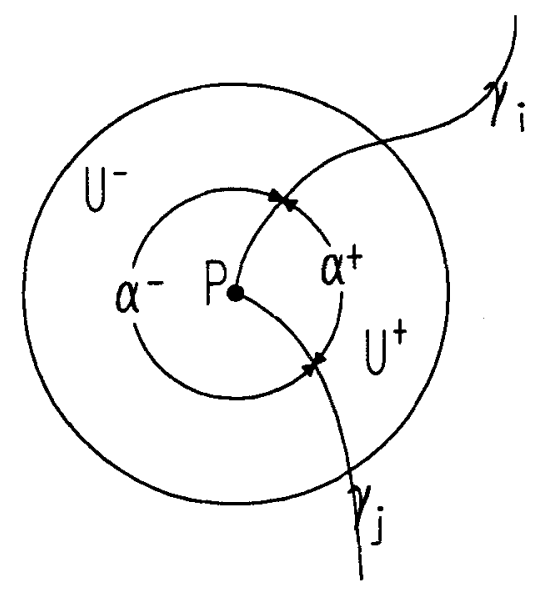

Figure 10. A corner in $\Gamma$. 
that $0 \leqq \eta \leqq 1$ and

$$
\eta(x, y)=\left\{\begin{array}{lll}
1 & \text { if } \quad x^{2}+y^{2} \geqq 1 \\
0 & \text { if } \quad x^{2}+y^{2} \leqq \frac{1}{2}
\end{array}\right.
$$

and let $\eta_{U}$ denote $\eta$ "adapted" to $U$, i.e.,

$$
\eta_{U}(x, y)=\eta\left(\frac{x-x(P)}{\varepsilon}, \frac{y-y(P)}{\varepsilon}\right)
$$

(so that $\eta_{U}=1$ outside $U, \eta_{U}=0$ in the concentric disc $U^{\prime}$ of radius $\frac{1}{2} \varepsilon$ ).

What we will do is to "cut" the corner at $P$ at a distance $\frac{1}{2} \varepsilon$ shrinking $U^{+}$ and expanding $U^{-}$; define a new $f$ on the new $U^{+}$by restriction; and define a new $f$ on the new $U^{-}$by $f^{-}(0)+\eta_{U}\left(f^{-}-f^{-}(0)\right)$. Let us estimate the change in $E(f, \Gamma)$. To make this estimate we can assume that $f^{-}(0)=0$ by replacing $f$ by $f-f^{-}(0), g$ by $g-f^{-}(0)$, which does not affect $E$. Then we have:

(a) Change in 1-st term:

$$
\begin{aligned}
& \iint_{U^{-}}\left(\eta_{U} f^{-}-g\right)^{2}-\iint_{U^{-}}\left(f^{-}-g\right)^{2} \\
& \quad=\iint_{U^{-}} \frac{1-\eta_{U}}{1+\eta_{U}} g^{2}-\left(1-\eta_{U}\right) \cdot\left(\sqrt{1+\eta_{U}} f^{-}-\frac{g}{\sqrt{1+\eta_{U}}}\right)^{2} \\
& \quad \leqq \iint_{U^{-}} g^{2} \\
& \quad \leqq c_{1} \varepsilon^{2} .
\end{aligned}
$$

(b) Change in 2-nd term:

$$
\begin{aligned}
\iint_{U^{-}} & \left\|\nabla\left(\eta_{U} f^{-}\right)\right\|^{2}-\iint_{U^{-}}\left\|\nabla f^{-}\right\|^{2} \\
& =\iint_{U^{-}}\left(f^{-}\right)^{2}\left\|\nabla \eta_{U}\right\|^{2}+2 \eta_{U} f^{-}\left(\nabla \eta_{U} \cdot \nabla f^{-}\right) .
\end{aligned}
$$

But $\left\|\nabla \eta_{U}\right\| \leqq c / \varepsilon$. Moreover, the estimates just discussed show that

$$
\begin{gathered}
f^{-}=O\left(r^{\pi / \alpha^{-}}\right), \\
\left\|\nabla f^{-}\right\|=O\left(r^{\left(\pi / \alpha^{-}\right)-1}\right) .
\end{gathered}
$$

Taking into account that $\nabla \eta_{U}=0$ outside the annulus between $\varepsilon$, $\frac{1}{2} \varepsilon$, we can 


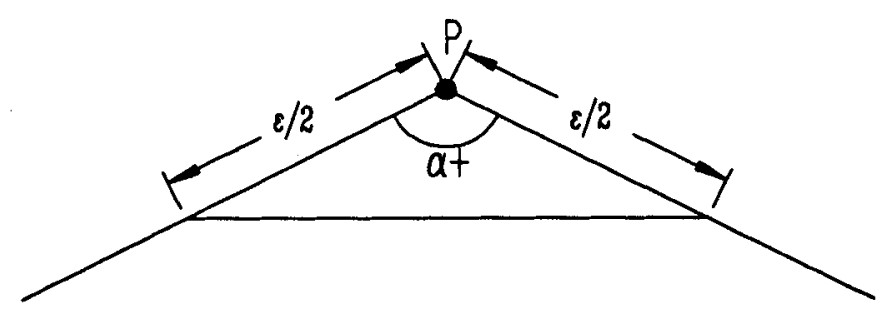

Figure 11. "Cutting" a corner on $\Gamma$.

bound the above expression:

$$
\begin{aligned}
& \leqq \frac{c^{2}}{\varepsilon^{2}} \underset{\text { Annulus }}{\sup }\left(f^{-}\right)^{2}\left(\frac{3}{4} \pi \varepsilon^{2}\right)+\frac{2 c}{\varepsilon} \sup _{\text {Annulus }}\left(\left|f^{-}\right|\left\|\nabla f^{-}\right\|\right)\left(\frac{3}{4} \pi \varepsilon^{2}\right) \\
& \leqq c_{1} \frac{1}{\varepsilon^{2}} \cdot \varepsilon^{2 \pi / \alpha^{-}} \cdot \varepsilon^{2}+c_{2} \frac{1}{\varepsilon} \cdot \varepsilon^{\left(\pi / \alpha^{-}+\pi / \alpha^{-}-1\right)} \cdot \varepsilon^{2} \\
& =c_{3} \varepsilon^{2 \pi / \alpha^{-}}
\end{aligned}
$$

(c) Change in 3-rd term:

Asymptotically, we are replacing the equal sides of an isosceles triangle with angle $\alpha^{+}$at apex by the 3-rd side (see Figure 11) which decreases the length term in $E$ from $\varepsilon$ to $\varepsilon \sin \left(\frac{1}{2} \alpha^{+}\right)$.

Note that terms 1 and 2 increase by order $\varepsilon^{2}$ and $\varepsilon^{2 \pi / \alpha^{-}}$, respectively (and $2 \pi / \alpha^{-}>1$ ), while term 3 decreases by order $\varepsilon$ which shows that if $\varepsilon$ is small, $E$ decreases.

The same argument gives us more results. Consider next points $P$ where an arc $\gamma_{i}$ of $\Gamma$ meets the boundary of the region $R$ where the problem is posed. At a minimum of $E$, we claim that $\gamma_{i}$ must meet $\partial R$ perpendicularly. Let $U$ be the intersection of a disc around $P$ of radius $\varepsilon$ and $R$, and suppose instead that $\gamma_{i}$ divides $U$ into a sector $U^{+}$with angle $\alpha^{+}<\frac{1}{2} \pi$ and a sector $U^{-}$with angle $\alpha^{-}>\frac{1}{2} \pi$ (see Figure 12). We modify $\Gamma$ and $f$ as follows: replace $\gamma_{i}$ by a new curve which, when it hits $U$, has a corner and follows a straight line meeting $\partial R$ perpendicularly; replace $f^{+}$by:its restriction, and $f^{-}$by $f^{-}(P)+\eta_{U} \cdot\left(f-f^{-}(P)\right)$, extended by $f^{-}(P)$. The above estimates show that the first two terms go up by order at most $\varepsilon^{2}$, while the 3-rd decreases by order $\varepsilon$, hence $E$ decreases.

Look next at triple points $P$ where three arcs $\gamma_{i}, \gamma_{j}, \gamma_{k}$ meet with angles $\alpha_{i j}$, $\alpha_{j k}, \alpha_{k i}$ (see Figure 13). We claim that, at a minimum of $E, \alpha_{i j}=\alpha_{j h}=\alpha_{k i}=\frac{2}{3} \pi$. If not, then one of the three angles is smaller than $\frac{2}{3} \pi$ : say $\alpha_{j k}<\frac{2}{3} \pi$ as in the diagram. Then modify $\Gamma$ by extending $\gamma_{i}$ along the bisector of angle $\alpha_{j k}$ and joining it by straight lines to the points where $\gamma_{j}$ and $\gamma_{k}$ first meet $U$ (see Figure 13). The new triple point is chosen so that its three angles are each $\frac{2}{3} \pi$. A little trigonometry will show that the old segments of $\gamma_{j}$ and $\gamma_{k}$ of total length 


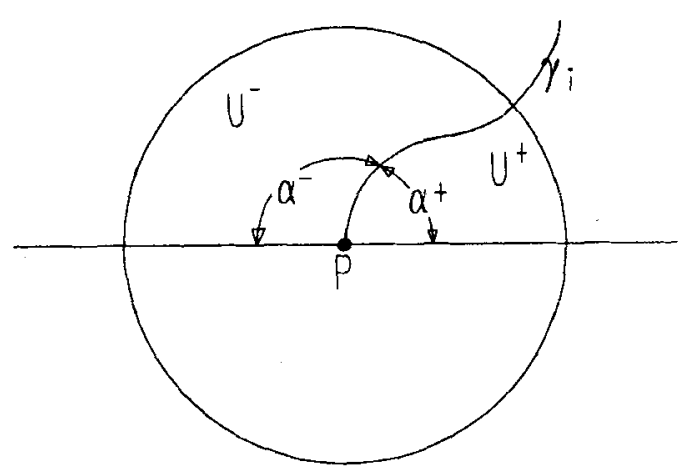

Figure 12. $\Gamma$ meets the boundary of $R$.

asymptotically equal to $2 \varepsilon$ are then replaced by three new straight segments of total length exactly

$$
2 \sin \left(\frac{1}{2} \alpha_{j k}+\frac{1}{6} \pi\right) \cdot \varepsilon
$$

which is clearly less than $2 \varepsilon$ if $\alpha_{j k}<\frac{2}{3} \pi$. This linear decrease in length is greater than the increase in the 1-st and 2-nd terms of $E$ by the same argument as before.

Finally, we can apply this argument to show that at a minimum of $E$ there are no points where four or more $\gamma_{i}$ meet at positive angles; simply choose the smallest angle $\alpha$ at the intersection. Then $\alpha \leqq \frac{1}{2} \pi$ and replace the 4 -fold intersection by two 3-fold intersections as in Figure 14.

In all of this, we have ignored "cuspidal corners", i.e., corners where the two arcs $\gamma_{i}$ and $\gamma_{j}$ are tangent. Our argument breaks down for cusps, but we can show that these do not occur by another argument. We cut off the end of one of

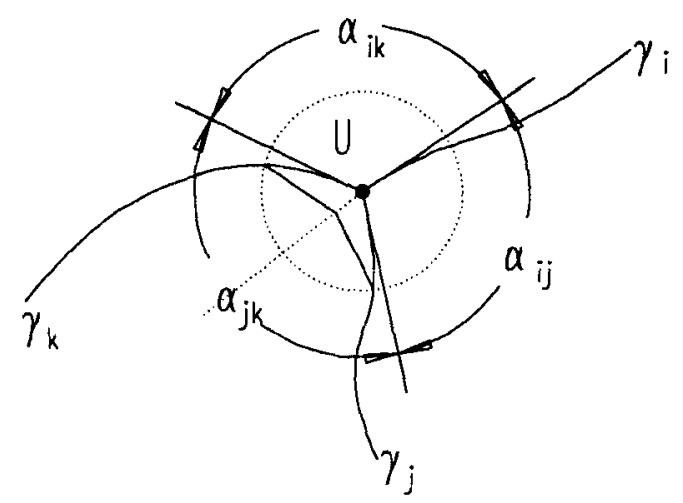

Figure 13. Modifying a triple point in $\Gamma$. 


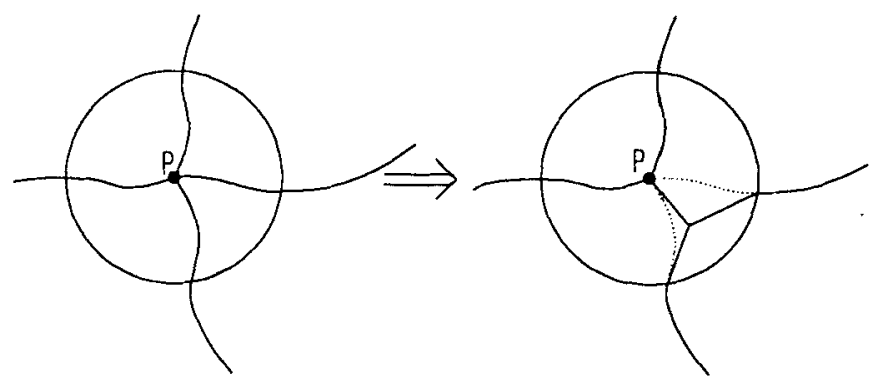

Figure 14. Splitting a 4-fold point into 2 triple points.

the arcs $\gamma_{i}$, join it to the nearest point of $\gamma_{j}$ and leave the end of $\gamma_{j}$ as a "crack-tip" (see Figure 15). To extend $f^{-}$from the old $U^{-}$to the new bigger $U^{-}=U-$ crack $\gamma_{j}$, we simply extend it from $\gamma_{i}$ by asking that $f^{-}$be constant on circles with center $P$. Again the length decreases by order $\varepsilon$. To check the increase in the 1-st and 2-nd terms, note the estimate on $f^{-}$:

$$
\begin{aligned}
f^{-} & =f^{-1}(0)+o(\sqrt{r}), \\
\left\|\nabla f^{-}\right\| & =O\left(\frac{1}{\sqrt{r}}\right) .
\end{aligned}
$$

Write $\gamma_{i}$ in polar coordinates as $\theta=\theta_{i}(r)$ and $\gamma_{j}$ by $\theta=\theta_{j}(r)$, so that $\theta_{i}-\theta_{j}=$ $O(r)$. Thus the new part of the second term is estimated like this:

$$
\begin{aligned}
\int_{0}^{\varepsilon} \int_{\theta_{i}}^{\theta_{j}}\left\|\nabla f^{-}\right\|^{2} r d \theta d r & \leqq \int_{0}^{\varepsilon} \frac{c}{r} \cdot r \cdot\left(\theta_{i}(r)-\theta_{j}(r)\right) d r \\
& \leqq c_{1} \varepsilon^{2} .
\end{aligned}
$$

This proves:

THEOREM 2.1. If $(f, \Gamma)$ is a minimum of $E$ such that $\Gamma$ is a finite union of simple $C^{1,1}$-curves meeting $\partial R$ and meeting each other only at their endpoints, then
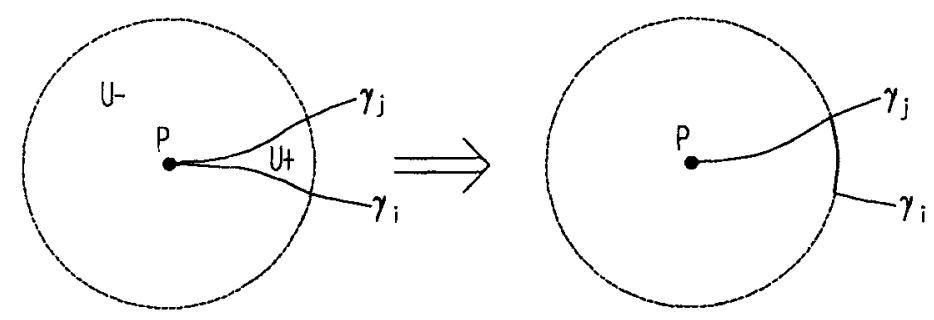

Figure 15. Eliminating cuspidal corners in $\Gamma$. 


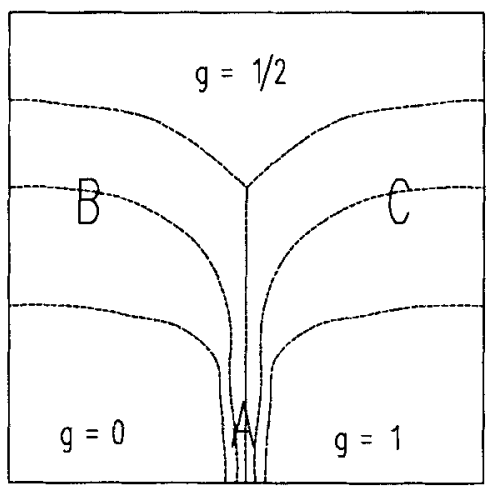

Figure 16. An image leading to a $\Gamma$ with a crack.

the only vertices of $\Gamma$ are:

(a) points $P$ on the boundary of $R$ where one $\gamma_{i}$ meets $\partial R$ perpendicularly,

(b) triple points $P$ where three $\gamma_{i}$ meet with angles $\frac{2}{3} \pi$,

(c) crack-tips $P$ where a $\gamma_{i}$ ends and meets nothing.

\section{Cuspidal Crack Tips}

Considering the original variational problem, we would first like to argue heuristically that "cracks", i.e., arcs in $\Gamma$ that end at a point $P$ without any continuation, are likely to occur in minima of $E$. Take $g$ to be a function of the type shown in Figure 16. If $\nu$ is set carefully, it will "pay" in terms of decreasing $E$ to have an arc of $\Gamma$ in strip $A$ separating the $g \equiv 0$ and $g \equiv 1$ regions but it will not pay to have arcs of $\Gamma$ in strips $B$ and $C$ : this is because $g$ has been concocted so that its gradient is much larger in strip $A$ than in $B$ or $C$; so putting $\Gamma$ along $A$ saves a big penalty in $\iint\|\nabla f\|^{2}$ but putting $\Gamma$ along $B$ or $C$ does not. We expect that in this case the optimal $\Gamma$ will run along $A$ to a crack-tip somewhere in the middle.

However, if $\|\nabla g\|$ is somewhat larger in $B$ than $C$, the crack will be expected to bend to the left into $B$ at its tip. Consider the rule which determines when $\Gamma$ is in balance between bending left or right:

$$
\text { (*) } \quad\left[\left(f_{+}-g\right)^{2}+\left\|\nabla f_{+}\right\|^{2}\right]-\left[\mu^{2}\left(f_{-}-g\right)^{2}+\left\|\nabla f_{-}\right\|^{2}\right]=\nu \cdot \operatorname{curv}(\Gamma)
$$

At the crack-tip itself, $\left(f_{ \pm}-g\right)^{2}$ is bounded, but $\left\|\nabla f_{ \pm}\right\|^{2}$ is not. $\left\|\nabla f_{ \pm}\right\|^{2}$ grows like $1 / r$, where $r$ is the distance to the crack-tip. Some cancellation takes place in $\left\|\nabla f_{+}\right\|^{2}-\left\|\nabla f_{-}\right\|^{2}$. Suppose for instance that the crack-tip is the positive $x$-axis 
and $f$ is approximated by

$$
f_{ \pm}= \pm c \mathscr{R} e \sqrt{z}+b \cdot x+\cdots
$$

(which satisfies $(\partial f / \partial n)_{ \pm}=\partial f / \partial y=0$ ). Then

$$
\left\|\nabla f_{+}\right\|^{2}-\left\|\nabla f_{-}\right\|^{2}=2 b c / \sqrt{r}+\cdots
$$

which still grows unboundedly near the crack-tip. Thinking of $(*)$ as an O.D.E., one would expect that this growth would, in general, force curv $(\Gamma)$ to grow like $1 / \sqrt{r}$ also. Curves that do this are curves with cusps at their endpoints:

$$
\Gamma: y=c \cdot x^{3 / 2}+\cdots, \quad x \geqq 0,
$$

where

$$
\operatorname{curv}(\Gamma)=\frac{y^{\prime \prime}}{\left(1+y^{\prime 2}\right)^{3 / 2}}=\frac{3 c}{4 \sqrt{x}}+\cdots
$$

For this reason, it seems logical to expect that crack-tips have cusps at their end. To confirm that such $\Gamma$ were consistent, we have made a careful calculation to produce functions $f$ and $g$ and a cuspidal crack $\Gamma$ satisfying the variational equations (*) and $\Delta f=\mu^{2}(f-g)$. In outline this goes as follows.

We work backwards:

(i) Start with $\Gamma$ given by

$$
y=x^{3 / 2}+\lambda_{1} x^{2}+\lambda_{2} x^{5 / 2}
$$$$
x \geqq 0,
$$

where $\lambda_{1}, \lambda_{2}$ will be chosen later.

(ii) Choose $f$ of the form

$$
f=f_{\text {sing }}+f_{\text {smooth }}
$$

where

$$
\begin{aligned}
f_{\text {sing }}= & 2 \sqrt{\nu} \frac{\sinh (\mu r)}{\mu \sqrt{r \pi}} \cos \left(\frac{1}{2} \theta\right) \\
& +\alpha \sqrt{\nu} \frac{3 \mu r \cosh (\mu r)-3 \sinh (\mu r)}{\mu^{2}(r)^{3 / 2}} \cdot \sin \left(\frac{3}{2} \theta\right)
\end{aligned}
$$

$\alpha$ will be chosen later, and $f_{\text {smooth }}$ is a $C^{\infty}$-function. It is easy to verify that

$$
\nabla^{2} f_{\text {sing }}=\mu^{2} \cdot f_{\text {sing }} .
$$


(iii) Choose

$$
g=f_{\text {smooth }}-\mu^{-2} \cdot \nabla^{2} f_{\text {smooth }} .
$$

Then by the choice of $f$ and $g$, it is clear that

$$
\Delta f=\mu^{2}(f-g) .
$$

Also, the leading term of $f$ is

$$
\frac{2 \sqrt{\nu}}{\sqrt{\pi}} \sqrt{r} \cos \left(\frac{1}{2} \theta\right) ;
$$

hence the extra variational condition, Griffith's law of cracks, derived later in this section is satisfied. We must check that for suitable $\lambda_{1}, \lambda_{2}, \alpha$ and suitable $f$ smooth, we also get

(a) $\partial f /\left.\partial n\right|_{\Gamma} \equiv 0$,

(b) $e_{+}-e_{-}=\nu \cdot \operatorname{curv}(\Gamma)$.

Taking into account that

$$
\begin{aligned}
& f_{+}=f_{\text {smooth }}+f_{\text {sing }}, \\
& f_{-}=f_{\text {smooth }}-f_{\text {sing }},
\end{aligned}
$$

and the definition of $g,(b)$ simplifies to

$$
4 \mu^{-2} \nabla^{2} f_{\text {smooth }} \cdot f_{\text {sing }}+4 \frac{\partial f_{\text {smooth }}}{\partial t} \cdot \frac{\partial f_{\text {sing }}}{\partial t}=\nu \cdot \operatorname{curv}(\Gamma)
$$

(where $\partial / \partial t$ is the directional derivative along $\Gamma$ ). The construction of $f_{\text {smooth }}$ is rather tedious and the values of the constants messy, but in outline, we may see that a solution is possible like this: Parametrize $\Gamma$ by $x$. Then $(1 / \sqrt{\nu})(\partial / \partial n) f_{\text {sing }}$ is a power series in $\sqrt{x}$ beginning with

$$
-\sqrt{\frac{1}{\pi}}-\frac{3}{2} \lambda_{1} \sqrt{\frac{x}{\pi}}+\cdots
$$

and $\operatorname{curv}(\Gamma)$ is a Laurent series in $\sqrt{x}$ beginning with

$$
\frac{3}{4 \sqrt{x}}+2 \lambda_{1}+\left(\frac{15}{4} \lambda_{2}-\frac{81}{32}\right) \sqrt{x}+\cdots
$$

and $(1 / \sqrt{\nu})(\partial / \partial t) f_{\text {sing }}$ is a Laurent series in $\sqrt{x}$ beginning with

$$
\sqrt{\frac{1}{\pi x}}-\frac{3}{4} \sqrt{\frac{x}{\pi}}+\cdots
$$


So we must solve the partial differential equations

$$
\begin{gathered}
\frac{1}{\sqrt{\nu}} \frac{\partial}{\partial n} f_{\text {smooth }}=\sqrt{\frac{1}{\pi}}+\frac{3}{2} \lambda_{1} \sqrt{\frac{x}{\pi}}+\cdots \\
\frac{4 \mu^{-2}}{\nu} \nabla^{2} f_{\text {smooth }}\left(\frac{2 \sqrt{\nu}}{\sqrt{\pi}} x+\cdots\right)+\frac{4}{\nu} \frac{\partial f_{\text {smooth }}}{\partial t}\left(\sqrt{\frac{\nu}{\pi}}+\cdots\right) \\
=\left(\frac{3}{4}+2 \lambda_{1} \sqrt{x}+\cdots\right) .
\end{gathered}
$$

Looking at low-order coefficients, it turns out that $f_{\text {smooth }}$ has three too few coefficients: in fact, if

$$
f_{\mathrm{smooth}}=\frac{3 \sqrt{\pi \nu}}{16} x+\sqrt{\frac{\mu \nu}{\pi}} y+\text { higher order terms }
$$

then the constant terms in (a) and ( $\left.b^{\prime}\right)$ are OK, but to make the $\sqrt{x}$-coefficients cancel we must set $\lambda_{1}=3 / \pi$ and $\alpha=3\left(1+\frac{1}{16} \pi^{2}\right) / \mu \cdot \pi^{3 / 2}$. Likewise the three quadratic coefficients in $f_{\text {smooth }}$ and $\lambda_{2}$ are needed to make the $x$ - and $x^{3 / 2}$. coefficients cancel. Thereafter the four terms $x^{n}, x^{n-1} y, x^{n-2} y^{2}$ and $x^{n-3} y^{3}$ in $f_{\text {smooth }}$ are sufficient to satisfy (a) and ( $\left.\mathrm{b}^{\prime}\right) \bmod x^{n}$. To see that convergence is not a problem, note that as soon as $f_{\text {smooth }}^{(0)}$ satisfies (a) and (b) $\bmod x^{3}$, we can set

$$
\begin{aligned}
f_{\text {smooth }}= & f_{\text {smooth }}^{(0)}+\left(y^{2}-x^{3}\right) \cdot\left(f_{1}(x)+y f_{2}(x)\right) \\
& +\left(y^{2}-x^{3}\right)^{2} \cdot\left(f_{3}(x)+y f_{4}(x)\right) .
\end{aligned}
$$

Substituting in (a), we solve for unique $f_{1}, f_{2}$ by dividing:

$$
f_{1}(x)+\left(x^{3 / 2}+\lambda_{1} x^{2}+\lambda_{2} x^{5 / 2}\right) f_{2}(x)=\frac{\left[\sqrt{1 / \pi}+\cdots-(\partial / \partial n) f_{\text {smooth }}^{(0)}\right]}{\left.(\partial / \partial n)\left(y^{2}-x^{3}\right)\right|_{\Gamma}}
$$

(noting that $\left.(\partial / \partial n)\left(y^{2}-x^{3}\right)\right|_{\Gamma}=2 x^{3 / 2}+\cdots$, so this is OK so long as $x^{3 / 2}$ divides the numerator). Substituting in (b), we solve similarly for $f_{3}$ and $f_{4}$, dividing this time by

$$
\left.\nabla^{2}\left(\left(y^{2}-x^{3}\right)^{2}\right)\right|_{\Gamma}=8 x^{3}+\cdots
$$

In Section 1, we derived extra variational equations at triple points and boundary points of $\Gamma$. There is also a new variational equation at crack-tips. It is the analogue of Griffiths' law of cracks well known in solid mechanics; see [12]. The difference is that in our case, cracks can be "sewn" back together as well as 
extended, hence we get an equality at critical points of $E$, not just an inequality. The result is this:

Theorem 3.1. Let $f, \Gamma$ be a critical point of $E$, and assume $\Gamma$ includes an arc ending at $P$ of the form

$$
y=a x^{3 / 2}+g(x), \quad g(x) \in C^{2}, \quad g(0)=g^{\prime}(0)=0
$$

in suitable coordinates $x, y$ where a may or may not be zero. Then if, near $P$,

$$
f(x, y)=a_{0}+a_{1} \mathscr{R} e(\sqrt{x+i y})+\cdots
$$

we must have

$$
\pi a_{1}^{2}=2 \nu
$$

To derive Griffiths' law of cracks and at the same time check for possible further conditions on a solution $(f, \Gamma)$ related to sideways perturbation of crack-tips, we use the general technique for deriving the first variation which is employed in geometric measure theory for highly singular $\Gamma$ 's. Let $X$ be a $C^{\infty}$-vector field on $R$, tangent to $\partial R$ and let $\Phi_{t}$ be the one-parameter group of diffeomorphisms from $R$ to $R$ obtained by integrating $X$. We want to compare $E\left(f \circ \Phi_{t}, \Phi_{t}^{-1}(\Gamma)\right)$ with $E(f, \Gamma)$ and, especially, compute

$$
\delta_{X} E(f, \Gamma)=\lim _{t \rightarrow 0} \frac{1}{t}\left[E\left(f \circ \Phi_{t}, \Phi_{t}^{-1}(\Gamma)\right)-E(f, \Gamma)\right] .
$$

For simplicity, we shall assume that $X$ is locally constant near crack-tips in $\Gamma$.

First, write

$$
\begin{aligned}
\delta_{X} E= & \mu^{2} \lim _{t \rightarrow 0} \int_{R}\left(\frac{f \circ \Phi_{t}-f}{t}\right) \cdot\left(f \circ \Phi_{t}+f-2 g\right) \\
& +\lim _{t \rightarrow 0} \frac{1}{t}\left[\iint_{R-\Phi_{t}^{-1}(\Gamma)}\left\|\nabla f \circ \Phi_{t}\right\|^{2}-\iint_{R-\Gamma}\|\nabla f\|^{2}\right] \\
& +\nu \cdot \lim _{t \rightarrow 0}\left[\frac{\text { length } \Phi_{t}^{-1} \Gamma-\text { length } \Gamma}{t}\right] .
\end{aligned}
$$

Call these terms $T_{1}, T_{2}$ and $T_{3}$. To evaluate the first integral $T_{1}$, let

$$
B_{r}=\bigcup_{0 \leqq s \leqq t} \Phi_{s}^{-1}(\Gamma)
$$


be the band swept out by $\Gamma$ while moving from $\Gamma$ to $\Phi_{t}^{-1}(\Gamma)$ and let

$$
h_{t}= \begin{cases}\left(f \circ \Phi_{t}-f\right) / t & \text { if } \quad(x, y) \notin B_{t}, \\ 0 & \text { if } \quad(x, y) \in B_{t} .\end{cases}
$$

We claim

$$
\begin{aligned}
\lim _{t \rightarrow 0} \iint_{R} h_{t} \cdot\left(f \circ \Phi_{t}+f-2 g\right) \\
\quad=\iint_{R} \lim _{t \rightarrow 0} h_{t} \cdot\left(f \circ \Phi_{t}+f-2 g\right) \\
\quad=\iint_{R} X f \cdot(2 f-2 g) .
\end{aligned}
$$

This follows from the Lebesgue bounded convergence theorem, plus the estimate:

LEMMA 3.2. Near a.crack-tip $P$,

$$
\left|h_{t}(x, y)\right| \leqq C / \sqrt{d}
$$

where $d$ is distance from $(x, y)$ to the line $\left\{\Phi_{t}(P) \mid t \in \mathbb{R}\right\}$.

Proof: The leading term in $f \circ \Phi_{t}$ is

$$
c \mathscr{R} e \sqrt{z-a t}
$$

where $z$ is a suitable complex coordinate centered at $P, a \in \mathbb{C}, c \in \mathbb{R}$. The corresponding term in $h_{t}$ is

$$
c \mathscr{R}_{\varepsilon}\left(\frac{\sqrt{z-a t}-\sqrt{z}}{t}\right)=c \mathscr{R}_{e} \frac{1}{\sqrt{z-a t}+\sqrt{z}} .
$$

But in $R-B_{t}$,

$$
|\sqrt{z-a t}+\sqrt{z}| \geqq|\sqrt{z}|
$$

(let $z=r_{1} \exp \left\{i \theta_{1}\right\}, z-a t=r_{2} \exp \left\{i \theta_{2}\right\}$. Then, if $\left|\theta_{1}-\theta_{2}\right| \leqq \pi$,

$$
|\sqrt{z-a t}+\sqrt{z}|^{2}=r_{1}+r_{2}+\sqrt{r_{1} r_{2}} \cos \left(\frac{1}{2}\left(\theta_{1}-\theta_{2}\right)\right) \geqq r_{1} .
$$

In $R-B_{t},\left|\theta_{1}-\theta_{2}\right| \leqq \pi$ (see Figure 17). Therefore the leading term in $h_{t}$ is bounded by $c / \sqrt{|z|}$, hence $c / \sqrt{d}$. The remainder $S$ in $f$ satisfies $S \in o\left(r^{1-\varepsilon}\right)$, 


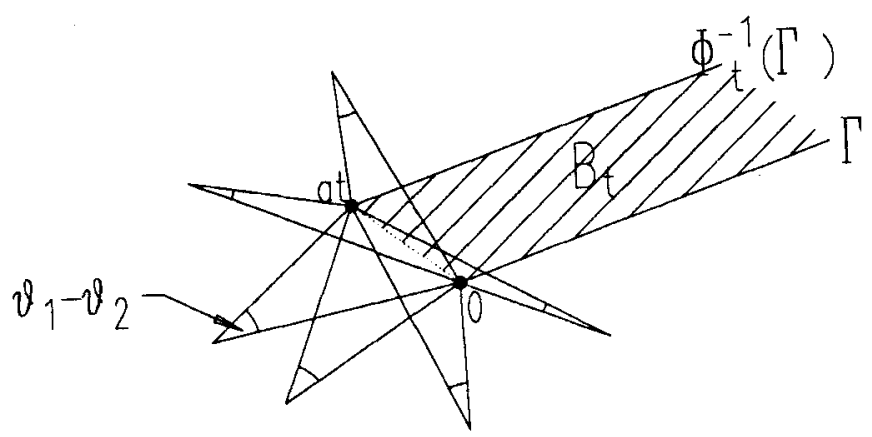

Figure 17. The function $\theta_{1}-\theta_{2}$.

$\|\nabla S\| \in o\left(r^{-\varepsilon}\right)$ for all $\varepsilon>0$, hence the remainder $S_{t}$ in $h_{t}$ satisfies

$$
\left|S_{t}(z)\right| \leqq \sup _{0 \leqq s \leqq t}\|\nabla R\|(z-a s) \leqq C d^{-\varepsilon} .
$$

This proves the lemma.

The rest of the first integral is

$$
\lim _{t \rightarrow 0} \frac{1}{t} \iint_{B_{t}}\left(f \circ \Phi_{t}-f\right)\left(f \circ \Phi_{t}+f-2 g\right)
$$

which equals

$$
\int_{\Gamma}\left(f_{+}-f_{-}\right)\left(f_{+}+f_{-}-2 g\right) d s
$$

( $f_{ \pm}=$boundary values of $f$ along $\Gamma$ ).

To evaluate the second integral $T_{2}$, let $D$ be a union of discs $D_{P}$ around all the crack-tips $P$ of $\Gamma$ of small radius $\delta$. Since $X$ is constant in $D, \Phi_{t}$ is a translation on $D$ and

$$
\iint_{\Phi_{t}^{-1}(D-\Gamma)}\left\|\nabla f \circ \Phi_{t}\right\|^{2}=\iint_{D-\Gamma}\|\nabla f\|^{2}
$$

Therefore the second integral equals:

$$
\begin{gathered}
\lim _{t \rightarrow 0} \frac{1}{t}\left[\iint_{R-\Phi_{t}^{-1} D-\Phi_{t}^{-1}(\Gamma)}\left\|\nabla f \circ \Phi_{t}\right\|^{2}-\iint_{R-D-\Gamma}\|\nabla f\|^{2}\right] \\
=\lim _{t \rightarrow 0} \iint_{R-D-\Gamma-\Phi_{t}^{-1}(\Gamma)} \frac{1}{t}\left(\left\|\nabla f \circ \Phi_{t}\right\|^{2}-\|\nabla f\|^{2}\right. \\
+\lim _{t \rightarrow 0} \frac{1}{t}\left[\iint_{\Phi_{t} D-D}\|\nabla f\|^{2}-\iint_{D-\Phi_{t} D}\|\nabla f\|^{2}\right]
\end{gathered}
$$


OPTIMAL APPROXIMATIONS

607

As above, define

$$
h_{t}(x, y)= \begin{cases}\frac{1}{t}\left(\left\|\nabla f \circ \Phi_{t}\right\|^{2}-\|\nabla f\|^{2}\right) & \text { if } \quad(x, y) \notin D \cup B_{t}, \\ 0 & \text { if } \quad(x, y) \in D \cup B_{t} .\end{cases}
$$

Then it is easy to check that

$$
\begin{aligned}
\lim _{t \rightarrow 0} \iint_{R-D} h_{t} & =\iint_{R-D} \lim _{t \rightarrow 0} h_{t} \\
& =2 \iint_{R-D}(\nabla(X f) \cdot \nabla f) \\
& =2 \int_{\partial D} X f \frac{\partial f}{\partial n} d s-2 \iint_{R-D} X f \cdot \Delta f,
\end{aligned}
$$

while

$$
\lim _{t \rightarrow 0} \iint_{B_{t}-B_{t} \cap D} \frac{1}{t}\left\|\nabla f \circ \Phi_{t}\right\|^{2}-\|\nabla f\|^{2}=\int_{\Gamma-\Gamma \cap D}\left(\left\|\nabla f_{+}\right\|^{2}-\left\|\nabla f_{-}\right\|^{2}\right) d s .
$$

But

$$
\begin{gathered}
\lim _{t \rightarrow 0} \frac{1}{t}\left(\iint_{\Phi_{t} D-D}\|\nabla f\|^{2}-\iint_{D-\Phi_{t} D}\|\nabla f\|^{2}\right) \\
=\sum_{P} \int_{\partial D_{P}}\|\nabla f\|^{2} \cos \left(\theta-\theta_{0}\right) \cdot r d \theta
\end{gathered}
$$

where $(r, \theta)$ are polar coordinates at $P$ and $\theta_{0}$ is the direction of $X(P)$. At each crack-tip $P$, write

$$
f=c_{P} \mathscr{R} e \sqrt{z}+S_{P}
$$

Then

$$
\|\nabla f\|^{2}=\frac{1}{4} c_{P}^{\dot{2}} \frac{1}{r}+S_{P}^{\prime}
$$

where $S_{P}^{\prime} \in o\left(r^{-1 / 2-e}\right)$. Therefore,

$$
\int_{\partial D_{P}}\|\nabla f\|^{2} \cos \left(\theta-\theta_{0}\right) \cdot r d \theta=\int_{\partial D_{P}} S_{P}^{\prime} \cos \left(\theta-\theta_{0}\right) \cdot r d \theta \leqq C \delta^{1 / 2-\varepsilon}
$$


Finally the third term $T_{3}$ is easily evaluated as

$$
\nu \int_{\Gamma} \kappa(P)\left(X(P) \cdot \vec{n}_{P}\right) d s+\sum_{\substack{\text { singular } \\ \text { points } \\ Q \text { of } \Gamma}}\left[\sum_{\substack{\text { branches } \\ \beta \text { of } \Gamma \\ \text { ending } \\ \text { at } Q}}\left(X(Q) \cdot \vec{t}_{\beta}\right)\right]
$$

where $\kappa(P)$ is the curvature of $\Gamma$ at $P, \vec{n}_{P}, \vec{t}_{P}$ are the unit normal and tangent vectors to $\Gamma$. Putting this together, we get

$$
\begin{aligned}
\delta_{X} E= & 2 \mu^{2} \iint_{R} X f \cdot(f-g)-2 \iint_{R-D} X f \cdot \Delta f \\
& +\mu^{2} \int_{\Gamma}\left(f_{+}-f_{-}\right)\left(f_{+}+f_{-}-2 g\right)+\int_{\Gamma-D}\left\|\nabla f_{+}\right\|^{2}-\left\|\nabla f_{-}\right\|^{2} \\
& +\nu \int_{\Gamma} \kappa d s+\sum_{\substack{\text { crack- } \\
\text { tips } P}} 2 \int_{\partial D_{P}} X f \frac{\partial f}{\partial n} d s \\
& +\sum_{\substack{\text { singular branches } \\
\text { points } \\
Q \text { of } \Gamma}} \sum_{\substack{\text { of } \Gamma\\
}} \nu\left(X_{Q} \cdot \vec{t}_{\beta}\right)+o(\delta) .
\end{aligned}
$$

Now let $\delta \rightarrow 0$. Use the fact that $\Delta f=\mu^{2}(f-g)$ on $R-\Gamma$, and that $e_{+}-e_{-}+$ $\nu \kappa \equiv 0$ along $\Gamma$, as proved in Section 1 . Moreover, at all singular points $Q$ other than crack-tips, it follows as before that either $Q$ is a triple point with $120^{\circ}$ angles, hence

$$
\sum_{\substack{b \text { at } Q \\ \beta \text { at } Q}} \overrightarrow{t_{\beta}}=\overrightarrow{0}
$$

or $Q$ is a point where $\Gamma$ meets $\partial R$ perpendicularly, hence $\left(X_{Q} \cdot \vec{t}_{\beta}\right)=0$. Thus all terms drop out as expected except for those at crack-tips, where we have

$$
\delta_{X} E=\sum_{\substack{\text { crack- } \\ \text { tips } P}} 2 \lim _{\delta \rightarrow 0} \int_{\partial D_{P}(\delta)} X(f) \frac{\partial f}{\partial n} d s+\nu\left(X_{P} \cdot \vec{t}_{P}\right)
$$

At each $P$, take coordinates such that $\Gamma$ is tangent to the positive $x$-axis, and 
write $f=C_{P} \mathscr{R} e \sqrt{z}+S_{P}$. Suppose $X=\alpha \partial / \partial x+\beta \partial / \partial y$. Then

$$
\begin{aligned}
X(f) & =\alpha \frac{C_{P}}{2 \sqrt{r}} \cos \left(\frac{1}{2} \theta\right)+\beta \frac{C_{P}}{2 \sqrt{r}} \sin \left(\frac{1}{2} \theta\right)+o\left(r^{-\varepsilon}\right) \\
\frac{\partial f}{\partial n} & =\frac{C_{P}}{2 \sqrt{r}} \cos \left(\frac{1}{2} \theta\right)+o\left(r^{-\varepsilon}\right)
\end{aligned}
$$

from which it follows easily that

$$
\lim _{\delta \rightarrow 0} \int_{\partial D_{P}} X(f) \frac{\partial f}{\partial n} d s=\frac{1}{4} \pi c_{P}^{2} \alpha
$$

\section{Approximation when $\mu$ is Small}

We derive here limiting forms of the energy and its first variation when $\mu$ is small. As before, let $f_{\Gamma}$ minimize $E(f, \Gamma)$ for fixed $\Gamma$. Let $R_{i}$ be the components of $R-\Gamma$ :

$$
R-\Gamma=R_{1} \sqcup \cdots \sqcup R_{n}
$$

and let

$$
\bar{g}_{\Gamma}=\left\{\begin{array}{l}
\text { function constant on each } R_{i} \\
\text { with value } \operatorname{mean}_{R_{i}}(g)
\end{array}\right.
$$

We shall prove that $f_{\Gamma}$ is very close to $\bar{g}_{\Gamma}$ when $\mu$ is small. Throughout this section, we assume that $\Gamma$ is a finite union of $C^{1,1}$-arcs meeting at corners with angles $\alpha, 0<\alpha<2 \pi$, or ending at crack-tips.

The error term depends on the smallest "necks" of each component $R_{i}$. For any region $W$, define the isoperimetric constant $h(W)$ by

$$
h(W)=\inf _{\gamma}\left\{\frac{|\gamma|}{\min \left(\left|W_{1}\right|,\left|W_{2}\right|\right)}: \begin{array}{c}
\gamma \text { is a curve dividing } W \\
\text { into } 2 \text { disjoint open } \\
\text { sets } W_{1} \text { and } W_{2}
\end{array}\right\}
$$

where $|\gamma|=$ length of $\gamma$ and $\left|W_{i}\right|=$ area of $W_{i}$ (compare Figure 5). Note that we have excluded cuspidal corners on the components $R_{i}$ of $R-\Gamma$ so $h\left(R_{i}\right)$ is positive. A bad case is shown in Figure 18. Let

$$
\lambda_{\Gamma}=\min _{i} h\left(R_{i}\right)
$$




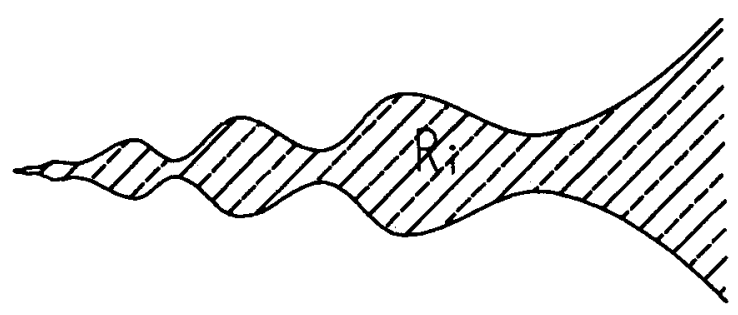

Figure 18. A domain with zero isoperimetric constant.

As in the introduction, we write

$$
E_{0}(\Gamma)=\iint_{R}\left(g-\bar{g}_{\Gamma}\right)^{2} d x d y+\nu_{0}|\Gamma|
$$

We now set $\nu_{0}=\nu / \mu^{2}$.

\section{THEOREM 4.1.}

$$
\mu^{2}\left\{E_{0}(\Gamma)-\frac{4 \mu^{2}}{\lambda_{\Gamma}^{2}+4 \mu^{2}}\|g\|_{0,2, R}^{2}\right\} \leqq E\left(f_{\Gamma}, \Gamma\right) \leqq \mu^{2} E_{0}(\Gamma)
$$

Proof: Let $f_{1}=f_{\Gamma}-\bar{g}_{\Gamma}$ and $g_{1}=g-\bar{g}_{\Gamma}$. Then $f_{1}$ satisfies the equation $\nabla^{2} f_{1}=\mu^{2}\left(f_{1}-g_{1}\right)$ in $R-\Gamma$ and homogeneous Neumann boundary condition along $\Gamma \cup \partial R$. By Green's identity for each component $W$ of $R-\Gamma$,

$$
\mu^{2} \int_{W} f_{1} g_{1}=\int_{W}\left(-\nabla^{2} f_{1}+\mu^{2} f_{1}\right) f_{1}=\int_{W}\left|\nabla f_{1}\right|^{2}+\mu^{2} \int_{W} f_{1}^{2}-\int_{\partial W} \frac{\partial f_{1}}{\partial n} f_{1}
$$

Since the last integral is zero,

(a) $\left\|\nabla f_{1}\right\|_{0,2, W}^{2}+\mu^{2}\left\|f_{1}\right\|_{0,2, W}^{2}=\mu^{2} \int_{W} f_{1} g_{1} \leqq \mu^{2}\left\|f_{1}\right\|_{0,2, W} \cdot\left\|g_{1}\right\|_{0,2, W}$

by Cauchy-Schwarz inequality. By Cheeger's inequality (see [3]),

$$
\inf _{\substack{u \neq 0 \\ \int_{W} u=0}} \frac{\|\nabla u\|_{0,2, W}^{2}}{\|u\|_{0,2, W}^{2}} \geqq \frac{1}{4} h^{2}(W)
$$


Since $\int_{W} g_{1}=0$ and, by Green's identity,

$$
\mu^{2} \int_{W}\left(f_{1}-g_{1}\right)=\int_{W} \nabla^{2} f_{1}=\int_{\partial W} \frac{\partial f_{1}}{\partial n}=0,
$$

we have

$$
\int_{W} f_{1}=0
$$

Therefore,

(b)

$$
\left\|\nabla f_{1}\right\|_{0,2, W}^{2} \geqq \frac{1}{4} h^{2}(W)\left\|f_{1}\right\|_{0,2, W}^{2} \geqq \frac{1}{4} \lambda_{\Gamma}^{2}\left\|f_{1}\right\|_{0,2, W}^{2} .
$$

Combining (a) and (b), we have

$$
\left\|f_{1}\right\|_{0,2, w} \leqq \frac{4 \mu^{2}}{\lambda_{\Gamma}^{2}+4 \mu^{2}}\left\|g_{1}\right\|_{0,2, w}
$$

and

$$
\left\|\nabla f_{1}\right\|_{0,2, W} \leqq \frac{2 \mu^{2}}{\sqrt{\lambda_{\Gamma}^{2}+4 \mu^{2}}}\left\|g_{1}\right\|_{0,2, w}
$$

Squaring these inequalities and summing over the components of $R-\Gamma$, we obtain the same inequalities with $W$ replaced by $R-\Gamma$. Finally,

$$
\begin{aligned}
0 & \leqq E\left(\bar{g}_{\Gamma}, \Gamma\right)-E\left(f_{\Gamma}, \Gamma\right)=\mu^{2} \int_{R} g_{1}^{2}-\mu^{2} \int_{R}\left(g_{1}-f_{1}\right)^{2}-\int_{R-\Gamma}\left|\nabla f_{1}\right|^{2} \\
& =2 \mu^{2} \int_{R} f_{1} g_{1}-\mu^{2} \int f_{1}^{2}-\int_{R-\Gamma}\left|\nabla f_{1}\right|^{2} \\
& =\mu^{2} \int_{R} f_{1} g_{1}, \\
& \leqq \mu^{2}\left\|f_{1}\right\|_{0,2, R}\left\|g_{1}\right\|_{0,2, R} \quad \text { by Green's identity as above, } \\
& \leqq \frac{4 \mu^{4}}{\lambda_{\Gamma}^{2}+4 \mu^{2}}\left\|g_{1}\right\|_{0,2, R}^{2} \\
& \leqq \frac{4 \mu^{4}}{\lambda_{\Gamma}^{2}+4 \mu^{2}}\|g\|_{0,2, R}^{2}
\end{aligned}
$$

so the theorem follows. 
We consider now the first variation of $E\left(f_{\Gamma}, \Gamma\right)$. Let $C_{0}^{1}(\Gamma)$ be the space of continuously differentiable functions on $\gamma$ which vanish in a neighborhood of the singular points of $\Gamma$. For each $\varphi \in C_{0}^{1}(\Gamma)$ and all sufficiently small $\tau>0$ define nearby curves $\Gamma_{\tau \varphi}$ as follows: let $(x(s), y(s))$ be a local parametrization of $\Gamma$ by arc length. Then $\Gamma_{T \varphi}$ is the curve

$$
s \mapsto(x(s), y(s))+\tau \varphi(s)\left(-y^{\prime}(s), x^{\prime}(s)\right) .
$$

THEOREM 4.2.

(i)

$$
\left.\frac{d E_{0}\left(\Gamma_{\tau \varphi}\right)}{d \tau}\right|_{\tau=0}=\int_{\Gamma}\left(\psi_{0}+\nu_{0} \kappa\right) \varphi d s
$$

where $\psi_{0}=\left(\bar{g}_{\Gamma}^{+}-\bar{g}_{\Gamma}^{-}\right)\left(\bar{g}_{\Gamma}^{+}+\bar{g}_{\Gamma}^{-}-2 g\right), \kappa=$ curvature of $\Gamma$. that

(ii) There exists a constant $C_{\Gamma}$ independent of $\mu$ (but which depends on $\Gamma$ ) such

$$
\left|\frac{d E\left(f_{\Gamma_{\tau \varphi}}, \Gamma_{\tau \varphi}\right)}{d \tau}\right|_{\tau=0}-\left.\mu^{2} \frac{d E_{0}\left(\Gamma_{\tau \varphi}\right)}{d \tau}\right|_{\tau=0} \mid \leqq C_{\Gamma} \mu^{4}\|\varphi\|_{0, \infty, \Gamma} \cdot\|g\|_{0, \infty, R}^{2} .
$$

Proof: Since $\bar{g}_{\Gamma}$ minimizes $E(f, \Gamma)$ over the space of locally constant functions over $R-\Gamma$, the first variation formula in part (i) follows in the same way as in the general case, derived in Section 1.

Define $f_{1}$ and $g_{1}$ as in the proof of Theorem 4.1. Let

$$
\begin{aligned}
\mathrm{e}_{1}(\Gamma) & \stackrel{\text { def }}{=} e\left(f_{\Gamma}\right)-e\left(\bar{g}_{\Gamma}\right) \\
& =\mu^{2}\left\{\left(g-f_{\Gamma}\right)^{2}-\left(g-\bar{g}_{\Gamma}\right)^{2}\right\}+\left|\nabla f_{\Gamma}\right|^{2} \\
& =\mu^{2}\left\{\left(g_{1}-f_{1}\right)^{2}-g_{1}^{2}\right\}+\left|\nabla f_{1}\right|^{2} \\
& =\mu^{2}\left(f_{1}^{2}-2 f_{1} g_{1}\right)+\left|\nabla f_{1}\right|^{2}
\end{aligned}
$$

The left-hand side in the inequality in part (ii) equals

$$
\left|\int_{\Gamma}\left(e_{1}^{+}(\Gamma)-e_{1}^{-}(\Gamma)\right) \varphi d s\right| \leqq \sum_{\substack{\text { components } \\ W \text { of } R-\Gamma}}\|\varphi\|_{0, \infty, \Gamma} \int_{\partial W}\left|e_{1}(\Gamma)\right| d s .
$$

We need to estimate $\left\|f_{1}\right\|_{1,2, \partial W}$. The boundary value problem

$$
\nabla^{2} u=v,\left.\quad \frac{\partial u}{\partial n}\right|_{\Gamma \cup \partial R}=0
$$


has a solution provided that $\int_{W} v=0$ for all components $W$ of $R-\Gamma$. The solution is unique if we require that $\int_{W} u=0$ for every $W$. In this case, since $\Gamma$ has no cuspidal corners, $u \in W_{p}^{2}(R-\Gamma)$ for every $p, 1 \leqq p<\frac{4}{3}$, by Appendix $1, \mathbf{H}$, and

$$
\|u\|_{2, p, R-\Gamma} \leqq C_{1}\|v\|_{0, p, R-\Gamma}
$$

where $C_{1}$ is a constant which depends on $\Gamma$. Applying this to the equation $\nabla^{2} f_{1}=\mu^{2}\left(f_{1}-g_{1}\right)$, we get

$$
\left\|f_{1}\right\|_{2, p, W} \leqq \mu^{2} C_{1}\left(\left\|f_{1}\right\|_{0, p, W}+\left\|g_{1}\right\|_{0, p, W}\right) .
$$

Since $L_{2}(W)$ embeds in $L_{p}(W)$ and we know from the proof of Theorem 4.1 that

$$
\left\|f_{1}\right\|_{0,2, W} \leqq \frac{4 \mu^{2}}{\lambda_{\Gamma}^{2}+4 \mu^{2}}\left\|g_{1}\right\|_{0,2, W}
$$

we have

$$
\left\|f_{1}\right\|_{2, p, W} \leqq \mu^{2} C_{2}\left\|g_{1}\right\|_{0,2, W}
$$

By the trace theorem (see [7]), the restriction of $u \in W_{p}^{2}(W)$ to $\partial W$ defines a continuous linear map

$$
W_{p}^{2}(W) \rightarrow W_{p}^{1}(\partial W) \subset W_{2}^{1}(\partial W) .
$$

Therefore there exists a constant $C_{3}$ depending on $\Gamma$ such that

$$
\left\|f_{1}\right\|_{1,2, \partial W} \leqq C_{3} \mu^{2}\left\|g_{1}\right\|_{0,2, W} .
$$

Going back to the energy density $e_{1}(\Gamma)$, we have

$$
\begin{aligned}
\int_{\partial W}\left|e_{1}(\Gamma)\right| d s & \leqq \mu^{2} \int_{\partial W} f_{1}^{2}+2 \mu^{2}\left(\int_{\partial W} f_{1}^{2}\right)^{1 / 2}\left(\int_{\partial W} g_{1}^{2}\right)^{1 / 2}+\int_{\partial W}\left|\nabla f_{1}\right|^{2} \\
& \leqq\left(\mu^{2}+1\right)\left\|f_{1}\right\|_{1,2, \partial W}^{2}+2 \mu^{2}\left\|f_{1}\right\|_{0,2, \partial W} \cdot\left\|g_{1}\right\|_{0,2, \partial W} \\
& \leqq C_{\Gamma} \mu^{4}\|g\|_{0, \infty, R}^{2} .
\end{aligned}
$$

\section{Existence of Solutions when $\mu=0$}

In case $\mu=0$, our free boundary value problem is not much more complicated than minimal-but singular-soap bubble problems. This is an especially easy case since we are dealing with singular sets $\Gamma$ which have dimension as well 
as codimension equal to one. The main result of this section is

Theorem 5.1. Let $R$ be an open rectangle ${ }^{4}$ in the plane and let $g$ be $a$ continuous function on $R \cup \partial R$. For all one-dimensional sets $\Gamma \subset R$ such that $\Gamma \cup \partial R$ is made up of a finite number of $C^{1.1}$-arcs, meeting each other only at their end-points, and, for all locally constant functions $f$ on $R-\Gamma$, let

$$
E_{0}(f, \Gamma)=\iint_{R}(f-g)^{2}+\nu_{0} \cdot \text { length }(\Gamma) .
$$

Then, there exist an $f$ and $a \Gamma$ which minimize $E_{0}$.

Geometric measure theory approaches problems of this sort by embedding them in larger minimizing problems in which extremely singular $\Gamma$ 's are allowed, then showing that in this larger world, a "weak solution" $\Gamma$ exists and finally arguing that any weak solution $\Gamma$ must be of the restricted type envisioned in the original formulation. As a weak version of our problem, we consider segmentations of $R$ by Cacciopoli sets which are measurable subsets of $R$ with finite "perimeter". Our standard reference for geometric measure theory is the book by L. Simon [13]. We let $L^{2}$ denote the Lebesgue measure on $\mathbb{R}^{2}$ and $H^{1}$ denote the one-dimensional Hausdorff measure on $\mathbb{R}^{2}$.

We begin by recalling De Giorgi's theory of Cacciopoli sets (see Section 14 in [13]). A bounded subset $F$ of $\mathbb{R}^{2}$ is called a Cacciopoli set if it is $L^{2}$-measurable and has finite "perimeter"; that is, the characteristic function $\chi_{F}$ of $F$ has bounded variation. For such a set $F$, there exists a Radon measure $\mu_{F}$ on $\mathbb{R}^{2}$ and a $\mu_{F}$-measurable function $\eta_{F}: \mathbb{R}^{2} \rightarrow \mathbb{R}^{2}$ with $\left|\eta_{F}\right|=1 \mu_{F}$-a.e. such that

$$
\int_{\mathbf{R}^{2}} \chi_{F} \operatorname{div}(\vec{g}) d L^{2}=-\int_{\mathbf{R}^{2}}\left(\vec{g} \cdot \eta_{F}\right) d \mu_{F}
$$

for all $C^{1}$-functions $\vec{g}: \mathbb{R}^{2} \rightarrow \mathbb{R}^{2}$ with compact support. We call $\mu_{F}$ the "generalized boundary measure" and $\eta_{F}$ the "generalized inward unit normal". Let $D \chi_{F}$ denote the gradient of $\chi_{F}$ in the sense of distributions. Then, $D \chi_{F}=\eta_{F} d \mu_{F}$ and $\eta_{F}, \mu_{F}$ can be recovered from $D \chi_{F}$ by

$$
\mu_{F}(U)=\sup _{\substack{|\vec{g}| \leqslant 1 \\ \text { supp }(\vec{g}) \subset U \\ \vec{g} \text { smooth }}} \int\left(D \chi_{F} \cdot \vec{g}\right) d L^{2}, \quad U \text { open, }
$$

and

$$
\eta_{F}(x)=\lim _{\rho \downarrow 0} \frac{\int_{B_{\rho}(x)} D_{\chi_{F}} d L^{2}}{\mu_{F}\left(B_{\rho}(x)\right)}
$$

\footnotetext{
${ }^{4}$ The restriction to a rectangle $R$ is not essential, but simplifies some technical aspects of the proof.
} 
where $B_{\rho}(x)$ denotes the ball of radius $\rho$, centered at $x$. The "perimeter" of $F$ is defined to be equal to $\mu_{F}\left(\mathbb{R}^{2}\right)$. Notice that if $F_{1}=F_{2} L^{2}$-a.e., then $\mu_{F_{1}}\left(\mathbb{R}^{2}\right)=$ $\mu_{F_{2}}\left(\mathbb{R}^{2}\right)$.

We may restate these results in the language of currents. Let $\vec{F}$ be the 2-current defined by $F$ :

$$
\vec{F}(\varphi)=\iint_{F} \varphi
$$

for all $C^{\infty}$ 2-forms $\varphi$ with compact support. Let $\partial \vec{F}$ denote the current boundary of $\vec{F}$ :

$$
\partial \vec{F}(\varphi)=\vec{F}(d \varphi)
$$

for all $C^{\infty} 1$-forms $\varphi$ with compact support. Then

$$
\partial \vec{F}(\varphi)=\int\left\langle\eta_{F}, \varphi\right\rangle d \mu_{F}
$$

For any set $S$, let $\partial S$ be the topological boundary $\bar{S}-\operatorname{Int}(S)$ of $S$. Then the topological boundary $\partial F$ of a Cacciopoli set $F$ may have positive $L^{2}$-measure and hence, infinite $H^{1}$-measure, even though $F$ still will have finite perimeter. Fortunately, it is possible to define the reduced boundary $\partial^{*} F$ so that the perimeter of $F$ equals the $H^{1}$-measure of $\partial^{*} F$ :

$$
\partial^{*} F=\left\{\begin{array}{ll}
x \in \mathbb{R}^{2}: & \begin{array}{c}
\eta_{F}(x) \text { as defined above exists } \\
\text { and has length } 1
\end{array}
\end{array}\right\}
$$

By De Giorgi's theorem (cf. [13], Section 14):

(i) $\partial^{*} F$ is 1-rectifiable.

(ii) $\mu_{F}=H^{1} L \partial^{*} F$ (i.e., $H^{1}$ restricted to $\partial^{*} F$ ).

(iii) For any set $S \subset \mathbb{R}^{2}, x \in \mathbb{R}^{2}, \rho>0$, let

$$
S_{x, \rho}=\left\{\rho^{-1}(y-x) \mid y \in S\right\}
$$

Then for every point $x \in \partial^{*} F$, the approximate tangent space $T_{x, \partial^{*} F}$ exists and is given by $\left\{\vec{y} \in \mathbb{R}^{2} \mid \vec{y} \cdot \eta_{F}(x)=0\right\}$, i.e.,

$$
\lim _{\rho \rightarrow 0} \int_{\left(\partial^{*} F\right)_{x, \rho}} f d H^{1}=\int_{\left(y \mid y \cdot \eta_{F}(x)=0\right\}} f d H^{1}
$$


for all continuous $f$ with compact support. Moreover,

$$
\lim _{\rho \rightarrow 0} \int_{F_{x, \rho}} f d L^{2}=\int_{\left\{y \mid y \cdot \eta_{F}(x)>0\right\}} f d L^{2}
$$

for all $f \in L^{1}\left(\mathbb{R}^{2}\right)$.

(iv) Rotating $\eta_{F}$ by $90^{\circ}$ defines a unit tangent vector $t_{F}$ to $\partial^{*} F$. Using $t_{F}$ to orient the 1-rectifiable set $\partial^{*} F$, we obtain a 1-current $\overrightarrow{\partial^{*} \vec{F}}$ such that

$$
\partial(\vec{F})=\overrightarrow{\left(\partial^{*} F\right)} \text {. }
$$

In particular, for all bounded open subsets $U \subset \mathbb{R}^{2}$, the mass $\mathbf{M}_{U}(\partial \vec{F})$ equals $H^{1}\left(U \cap \partial^{*} F\right)$.

We next reformulate $E_{0}$ using Cacciopoli sets. Note that if $\Gamma$ has an arc $\gamma$ which is surrounded by a single component of $R-\Gamma$, we can reduce the energy $E_{0}$ simply by removing $\gamma$. Hence, we might as well assume in our original formulation that the boundary of each component of $R-\Gamma$ consists of piecewise $C^{1,1}$-loops which mutually intersect in only finitely many points (see Figure 19). Therefore, for each component $F$ of $R-\Gamma$, and each arc $\gamma$ in $\partial F, \gamma$ is the boundary of $F$ from only one side; hence using the notion of the mass of a current:

$$
\text { length }(\partial F)=\mathbf{M}(\partial F) \text {. }
$$

Thus

$$
2 \text { length }(\Gamma)+\text { length }(\partial R)=\sum_{\left(\begin{array}{c}
\text { conn. comp. } \\
F \text { of } R-\Gamma
\end{array}\right)} \mathbf{M}(\partial F)
$$

and

$$
\text { length }(\Gamma)=\frac{1}{2} \sum_{\substack{\text { conn. comp. } \\ F \text { of } R-\Gamma}} \mathbf{M}_{R}(\partial F)
$$

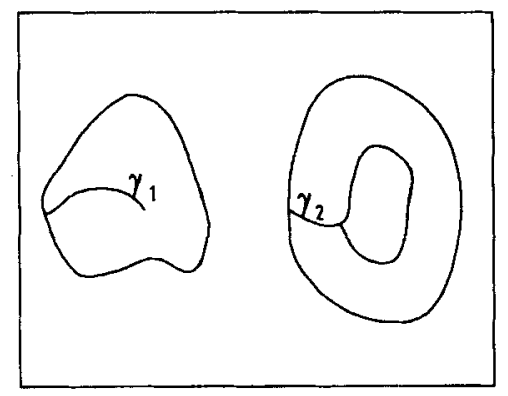

Figure 19. Removable arcs $\gamma_{i}$. 
This motivates the following new functional: fix some positive integer $n$, and consider sets of $n$ Cacciopoli sets, $U_{1}, U_{2}, \cdots, U_{n}$, such that

$$
U_{i} \subset R \text { for } 1 \leqq i \leqq n,
$$

and

$$
\sum_{i=1}^{n} \vec{U}_{i}=\vec{R}
$$

For such $\left\{U_{i}\right\}$ and for all constants $a_{1}, \cdots, a_{n}$, define

$$
E_{n}\left(\left\{U_{i}, a_{i}\right\}\right)=\sum_{i}\left[\frac{1}{2} \nu_{0} \mathbf{M}_{R}\left(\partial \vec{U}_{i}\right)+\int_{U_{i}}\left(g-a_{i}\right)^{2} d L^{2}\right] .
$$

This is our "weak" formulation. Note that to make a reasonable "weak" problem, we have to fix $n$. However, the $U_{i}$ are required to be neither connected nor nonempty. Hence,

$$
\inf _{\left\{U_{i}, a_{i}\right\}} E_{n^{\prime}} \leqq \inf _{\left\{U_{i}, a_{i}\right\}} E_{n} \text { if } \quad n^{\prime} \geqq n .
$$

We shall show:

THEOREM 5.2. (a) For each $n \geqq 1, E_{n}$ takes on a minimum value for some $\left\{U_{i}, a_{i}\right\}$.

(b) If $\left\{U_{i}, a_{i}\right\}$ minimizes $E_{n}$, then each $U_{i}$ is an open set with a finite number of components and piecewise $C^{2}$ boundary.

(c) There exists an integer $n_{0}$ such that

$$
\min _{\left\{U_{i}, a_{i}\right\}} E_{n}=\min _{\left\{U_{i}, a_{i}\right\}} E_{n_{0}} \text { for all } n \geqq n_{0} \text {. }
$$

In fact,

$$
E_{n}\left(\left\{U_{i}, a_{i}\right\}\right)>\min E_{n_{0}}
$$

if $n>n_{0}$ and each $U_{i}$ is nonempty.

Clearly, this will prove Theorem 5.1. Now consider Theorem 5.2. Part (a) is an immediate consequence of the compactness theorem for functions of bounded variation (see [13], Section 6). In fact, if $\left\{U_{i}^{\alpha}, a_{i}^{\alpha}\right\}$ is a minimizing sequence so that

$$
\lim _{\alpha} E_{n}\left(\left\{U_{i}^{\alpha}, a_{i}^{\alpha}\right\}\right)=\inf E_{n},
$$

then, by the compactness theorem, for each $i$, there exists a subsequence of $\left\{U_{i}^{\alpha}\right\}$ 
which converges to a Cacciopoli set $U_{i}$ (i.e., the integral of any $L^{1}\left(\mathbb{R}^{2}\right)$ function on $U_{i}^{\alpha}$ converges to its integral on $U_{i}$ ) such that

$$
H^{1}\left(\partial^{*} U_{i}\right) \leqq \liminf _{\alpha} H^{1}\left(\partial^{*} U_{i}^{\alpha}\right)
$$

Moreover, minimizing with respect to $a_{i}$ is seen immediately to mean

$$
a_{i}=\frac{1}{L^{2}\left(U_{i}\right)} \int_{U_{i}} g d L^{2}
$$

Hence, we may assume that $0 \leqq a_{i}^{\alpha} \leqq \max |g|$ for all $i$ and $\alpha$. Therefore, on a suitable subsequence, all $U_{i}^{\alpha}$ and $a_{i}^{\alpha}$ converge, and

$$
\inf E_{n} \leqq E_{n}\left(\left\{U_{i}, a_{i}\right\}\right) \leqq \liminf _{\alpha} E_{n}\left(\left\{U_{i}^{\alpha}, a_{i}^{\alpha}\right\}\right)=\inf E_{n} .
$$

The identity $\sum_{i=1}^{n} \vec{U}_{i}^{\alpha}=\vec{R}$ passes to the limit so that $\sum_{i=1}^{n} \vec{U}_{i}=\vec{R}$.

Part (b) is the hardest to prove. Our method is essentially a generalization of the theory of minimizing currents of codimension 1 . In outline, the proof is as follows: Let

$$
\Gamma^{*}=\bigcup_{i=1}^{n}\left(R \cap \partial^{*} U_{i}\right)
$$

$\Gamma^{*}$ is the weak version of the set of curves $\Gamma$ specified in Theorem 5.1. We rewrite $E_{n}$ in terms of $\Gamma^{*}$ and then consider the first variation of $E_{n}$ with respect to $\Gamma^{*}$. This shows that $\Gamma^{*}$ has generalized mean curvature and hence the monotonicity formula of geometric measure theory applies. We conclude that if $\left\{U_{i}, a_{i}\right\}$ minimizes $E_{n}$, then $\Gamma^{*}$ equals its closure $\Gamma H^{1}$-a.e. in $R$. Hence, we may assume that the $U_{i}$ are open and $\Gamma$ is the union of their topological boundaries in $R$. We next study the singularities of $\Gamma$. To do this, we first show that tangent cones exist everywhere on $\Gamma$ and that they have multiplicity away from the origin. We conclude that the singularity set of $\Gamma$ is discrete. Allard's theorem implies that $\Gamma$ is $C^{2}$ away from singularities. The rest of the proof now follows easily.

Before giving the details of the proof, we introduce a construction which will be used several times in the proof to handle the behavior of $\Gamma^{*}$ along $\partial R$. This is to consider a larger region $R^{*}$ built out of four copies of $R$ centered around one of the corners $P$ of $R$; see Figure 20. The function $g$ is extended to a continuous function $g^{\#}$ on $R^{\#}$ by reflection. Thus suppose $P=(0,0)$ after a translation. Then in the situation of the figure

$$
g^{\#}(x, y)=\left\{\begin{array}{lll}
g(x, y) & \text { in } R_{1}, \\
g(-x, y) & \text { in } R_{3}, \\
g(x,-y) & \text { in } R_{2}, \\
g(-x,-y) & \text { in } R_{4} .
\end{array}\right.
$$




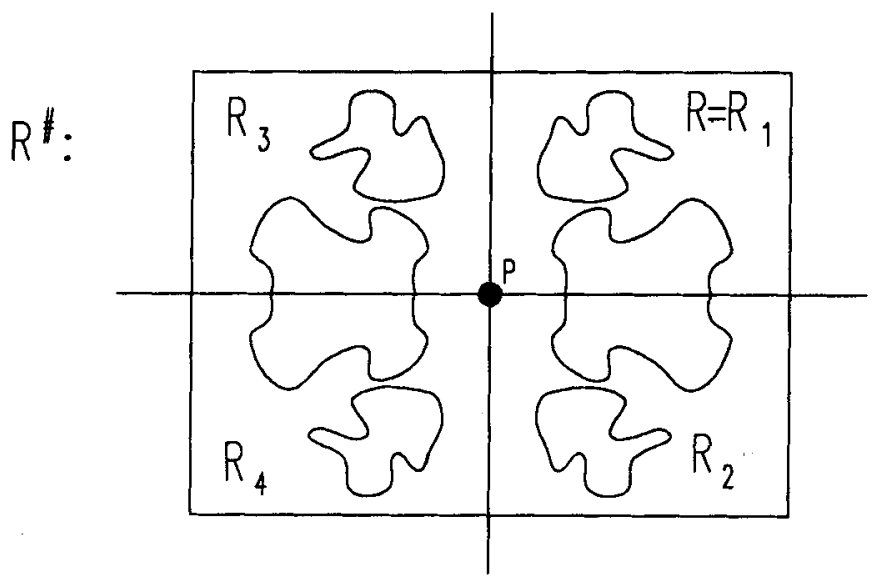

Figure 20. A segmentation reflected around a corner $P$.

Define $U_{i}^{\#}$ to be the union of $U_{i}$ and its three mirror images in $R^{\#}$. Note that $\mathbf{M}_{R^{*}}\left(\partial \vec{U}_{i}^{\#}\right)=4 \mathbf{M}_{R}\left(\partial \vec{U}_{i}\right)$. In fact,

$$
R^{\#}=R_{1} \cup R_{2} \cup R_{3} \cup R_{4} \cup \Delta,
$$

where $\Delta$ is the part of the horizontal and vertical axes through $P$ which is in $R^{\#}$. Then

$$
\mathbf{M}_{R^{*}}\left(\partial \vec{U}_{i}^{\#}\right)=\sum_{\alpha=1}^{4} \mathbf{M}_{R_{\alpha}}\left(\partial \vec{U}_{i}^{\#}\right)+H^{1}\left(\partial^{*} U_{i}^{*} \cap \Delta\right)
$$

But

$$
\partial^{*} \vec{U}_{i}^{\#}=\partial \vec{U}_{i}^{(1)}+\partial \vec{U}_{i}^{(2)}+\partial \vec{U}_{i}^{(3)}+\partial \vec{U}_{i}^{(4)}
$$

and if $\partial^{*} U_{i}^{\#}$ were to include a portion of $\Delta$ of positive $H^{1}$-measure, it would occur in two boundaries $\partial^{*} U_{i}^{(\alpha)}$, but with the opposite normal vectors $\eta_{i}^{(\alpha)}$. Thus it would have to cancel out in $\partial\left(\vec{U}_{i}^{\#}\right)$. This shows that in fact

$$
E_{n}\left(\left\{U_{i}^{\#}, a_{i}\right\}\right)=4 E_{n}\left(\left\{U_{i}, a_{i}\right\}\right) .
$$

If $\left\{U_{i}, a_{i}\right\}$ minimizes $E_{n}$ and if $\left\{V_{i}, b_{i}\right\}$ is any other decomposition of $R^{\#}$, then

$$
\begin{aligned}
E_{n}\left(\left\{V_{i}, b_{i}\right\}\right) & \geqq \sum_{\alpha=1}^{4} E_{n}\left(\left\{V_{i} \cap R_{\alpha}, b_{i}\right\}\right) \\
& \geqq 4 E_{n}\left(\left\{U_{i}, a_{i}\right\}\right) \\
& =E_{n}\left(\left\{U_{i}^{\#}, a_{i}\right\}\right) ;
\end{aligned}
$$


hence $\left\{U_{i}^{\#}, a_{i}\right\}$ minimizes $E_{n}$ for $g^{\#}$ on $R^{\#}$. Finally, the curve $\Gamma^{\#, *}$ defined by $\left\{U_{i}^{\#}, a_{i}\right\}$ is obtained from $\Gamma^{*}$ and its three reflections and a set of $H^{1}$-measure zero in $\Delta$.

We now begin the detailed proof.

LEMMA 5.3. Let $F_{1}, F_{2}$ be Cacciopoli sets. Let $F=F_{1} \cup F_{2}$ and assume that $\vec{F}=\vec{F}_{1}+\vec{F}_{2}$. Then,

$$
\partial^{*} F=\partial^{*} F_{1} \cup \partial^{*} F_{2}-\partial^{*} F_{1} \cap \partial^{*} F_{2} \quad H^{1} \text {-a.e. }
$$

Hence, for all open bounded subsets $U \subset \mathbb{R}^{2}$,

$$
\begin{aligned}
H^{1}\left(U \cap \partial^{*} F\right)= & {\left[H^{1}\left(U \cap \partial^{*} F_{1}\right)-H^{1}\left(U \cap \partial^{*} F_{1} \cap \partial^{*} F_{2}\right)\right] } \\
& +\left[H^{1}\left(U \cap \partial^{*} F_{2}\right)-H^{1}\left(U \cap \partial^{*} F_{1} \cap \partial^{*} F_{2}\right)\right] .
\end{aligned}
$$
show

Proof: Let $M$ denote $\partial^{*} F_{1} \cup \partial^{*} F_{2}$ and $N$ denote $\partial^{*} F_{1} \cap \partial^{*} F_{2}$. We shall

(i) $N \cap \partial^{*} F$ is empty,

(ii) $M-N \subset \partial^{*} F \quad H^{1}$-a.e.,

(iii) $\partial^{*} F \subset M-N \quad H^{1}$-a.e.

(i) Let $x \in N$. For $\rho>0$, let

$$
\left(F_{i}\right)_{x, \rho}=\left\{\rho^{-1}(y-x): y \in F_{i}\right\} \text { for } i=1,2 \text {, }
$$

and

$$
F_{x, \rho}=\left\{\rho^{-1}(y-x): y \in F\right\}
$$

By De Giorgi's theorem, $\lim _{\rho \downarrow 0}\left(F_{i}\right)_{x, \rho}=H_{i}$, where $H_{i}$ is an open half-space in $\mathbb{R}^{2}$. $H_{1}$ and $H_{2}$ must be disjoint. For, if $H_{1} \cap H_{2}$ is non-empty, there exists a ball $B \subset H_{1} \cap H_{2}$ and $f \in C_{c}^{\infty}(B)$ such that, for $i=1,2$,

$$
\lim _{\rho \downarrow 0} \int_{\left(F_{i}\right)_{x, \rho}} f d L^{2}=\int_{H_{i}} f d L^{2}>0
$$

Since $\vec{F}=\vec{F}_{1}+\vec{F}_{2}$,

$$
\begin{aligned}
\lim _{\rho \downarrow 0} \int_{F_{x, \rho}} f d L^{2} & =\lim _{\rho \downarrow 0} \int_{\left(F_{1}\right)_{x, \rho}} f d L^{2}+\lim _{\rho \downarrow 0} \int_{\left(F_{1}\right)_{x, \rho}} f d L^{2} \\
& =2 \int_{\mathbf{R}^{2}} f d L^{2} .
\end{aligned}
$$


Therefore, there exists $\rho>0$ such that

$$
\int_{F_{x, p}} f d L^{2}>\int_{\mathbf{R}^{2}} f d L^{2}
$$

which is absurd. It follows that $H_{1}$ and $H_{2}$ are complementary half-spaces; hence $\lim _{\rho \downarrow 0} F_{x, \rho}=\mathbb{R}^{2}$ and thus $x \notin \partial^{*} F$ by De Giorgi's theorem.

(ii) Let $x \in M-N$, say $x \in \partial^{*} F_{1}-N$. Outside a set of $H^{1}$-measure zero, we may assume that the upper density $\Theta^{*}\left(H^{1}, \partial^{*} F_{2}, x\right)=0$. Write out $\eta_{F}(x)$ :

$$
\eta_{F}(x)=\lim _{\rho \downarrow 0} \frac{\int_{B_{\rho}(x)} D \chi_{F} d L^{2}}{\mu_{F}\left(B_{\rho}(x)\right)}=\frac{\lim _{\rho \downarrow 0}(1 / 2 \rho) \int_{B_{\rho}(x)} D \chi_{F} d L^{2}}{\Theta\left(H^{1}, \partial^{*} F, x\right)} .
$$

Since $\Theta\left(H^{1}, \partial^{*} F_{1}, x\right)=1$ and $\Theta^{*}\left(H^{1}, \partial^{*} F_{2}, x\right)=0$,

$$
\Theta\left(H^{1}, \partial^{*} F, x\right)=\lim _{\rho \downarrow 0} \frac{1}{2 \rho} \mu_{F}\left(B_{\rho}(x)\right)=1 .
$$

Since $D \chi_{F}=D \chi_{F_{1}}+D \chi_{F_{2}}$ it also follows that

$$
\lim _{\rho \downarrow 0} \frac{1}{2 \rho} \int_{B_{\rho}(x)} D \chi_{F} d L^{2}=\lim _{\rho \downarrow 0} \frac{1}{2 \rho} \int_{B_{\rho}(x)} D \chi_{F_{1}} d L^{2}=\eta_{F_{1}}(x) .
$$

Hence $\eta_{F}(x)$ exists and has length 1 , i.e., $x \in \partial^{*} F$.

(iii) If $x \in \partial^{*} F-M$, we may assume that

$$
\Theta^{*}\left(H^{1}, \partial^{*} F_{i}, x\right)=0 \text { for } i=1,2 \text {. }
$$

Then, $\Theta^{*}\left(H^{1}, \partial^{*} F, x\right) \leqq \Theta^{*}\left(H^{1}, \partial^{*} F_{1}, x\right)+\Theta^{*}\left(H^{1}, \partial^{*} F_{2}, x\right)=0$ and so $H^{1}\left(\partial^{*} F-M\right)=0$.

LEMMA 5.4. Let $U_{1}, U_{2}, \cdots, U_{n}$ be Cacciopoli sets such that $\sum_{i=1}^{n} \vec{U}_{i}=\vec{R}$. Then,

(a) for $H^{1}$-a.e., $x \in \Gamma^{*}$ belongs to precisely two $\partial^{*} U_{i}$ 's,

(b) $H^{1}\left\llcorner\Gamma^{*}=\frac{1}{2} \sum_{i=1}^{n} H^{1} L\left(R \cap \partial^{*} U_{i}\right)\right.$.

In particular,

$$
H^{1}\left(\Gamma^{*}\right)=\frac{1}{2} \sum_{i=1}^{n} \mathbf{M}_{R}\left(\partial \vec{U}_{i}\right)
$$

Proof: (a) Suppose $x \in R \cap \partial^{*} U_{i}$ for some $i$. Let $U=U_{j \neq i} U_{j}$. Since $\vec{U}_{i}=$ $\vec{R}-\vec{U}, \partial \vec{U}_{i}=\partial \vec{R}-\partial \vec{U}$ and hence

$$
R \cap \partial^{*} U_{i}=R \cap \partial^{*} U \quad H^{1} \text {-a.e. }
$$


By Lemma 5.3,

$$
\begin{aligned}
R \cap \partial^{*} \dot{U}= & \bigcup_{j \neq i}\left(R \cap \partial^{*} U_{j}\right) \\
& -\left\{y: y \in\left(\partial^{*} U_{j}\right) \cap\left(\partial^{*} U_{k}\right) \cap R \text { for } j, k \neq i\right\} \quad H^{1} \text {-a.e. } \\
= & \left\{y: y \in R \cap \partial^{*} U_{j} \text { for some unique } j \neq i\right\} \quad H^{1} \text {-a.e. }
\end{aligned}
$$

(b) Let $\mu=H^{1}\left\llcorner\Gamma^{*}\right.$ and $\mu_{i}=H^{1} L\left(R \cap \partial^{*} U_{i}\right)$ :

$$
\begin{aligned}
D_{\mu} \sum_{i=1}^{n} \mu_{i}(x) & =\lim _{\operatorname{def}} \frac{\sum_{i=1}^{n} \mu_{i}\left(B_{\rho}(x)\right)}{\mu\left(B_{\rho}(x)\right)} \\
& =\frac{\sum_{i=1}^{n} \Theta\left(\mu_{i}, x\right)}{\Theta(\mu, x)} \\
& =2 \quad H^{1} \text {-a.e. }
\end{aligned}
$$

by part (a).

Part (b) now follows from Theorem 4.7 in [13].

LEMMA 5.5. Let $\left\{U_{i}, a_{i}\right\}$ minimize $E_{n}$. Let $\Gamma=$ closure of $\Gamma^{*}$ in $\bar{R}$. Let $\eta_{i}$ denote the generalized inward unit normal in $R$ corresponding to the Cacciopoli set $U_{i}$. Letting $\eta_{i}$ be zero on $\Gamma^{*}-\partial^{*} U_{i}$, define $\kappa: \Gamma^{*} \rightarrow \mathbb{R}^{2}$ by

$$
\kappa=-\frac{1}{\nu_{0}} \sum_{i=1}^{n}\left(g-a_{i}\right)^{2} \eta_{i}
$$

Then,

(a) $\Gamma^{*}$ has the generalized curvature $\kappa$. That is, for all $C^{1}$-vector fields $\mathbf{X}$ on $\bar{R}$ tangent to $\partial R$ along $\partial R$, if $D X$ is the $2 \times 2$ matrix of derivatives of the components of $\mathbf{X}$ and if $t: \Gamma^{*} \rightarrow \mathbb{R}^{2}$ is a unit tangent vector (with any choice of signs),

$$
\int_{\Gamma^{*}}\left(t^{\prime} \cdot D \mathbf{X} \cdot t\right) d H^{1}=-\int_{\Gamma^{*}}(\kappa \cdot \mathbf{X}) d H^{1}
$$

(b) Monotonicity: Define a function $\varepsilon$ on $\bar{R}$ by

$$
\varepsilon(x)=\left\{\begin{array}{lll}
1 & \text { if } & x \in R \\
2 & \text { if } & x \in \partial R, \text { but } x \text { is not a corner } \\
4 & \text { if } & x \text { is a corner of } \bar{R}
\end{array}\right.
$$


Then the density $\Theta\left(H^{1}, \Gamma^{*}, x\right)$ exists for all $x \in \Gamma, \varepsilon \Theta$ is greater than or equal to 1 , and is upper semi-continuous.

(c) $\Gamma=\Gamma^{*} \quad H^{1}$-a.e. so that $\frac{1}{2} \sum_{i-1}^{n} \mathbf{M}_{R}^{\prime}\left(\partial \vec{U}_{i}\right)=H^{1}(\Gamma)$.

(d) We may assume that the $U_{i}$ are open and that $\Gamma$ equals the union of their topological boundaries in $R$.

Proof: (a) By Lemma 5.4,

$$
E_{n}=\nu_{0} H^{1}\left(\Gamma^{*}\right)+\sum_{i=1}^{n} \int_{U_{i}}\left(g-a_{i}\right)^{2} d L^{2} .
$$

Consider the first variation $\delta_{\mathbf{x}} E_{n}$ of $E_{n}$ with respect to a $C^{1}$-vector field $\mathbf{X}$ on $\bar{R}$, tangent to $\partial R$ along $\partial R$. The formula

$$
\delta_{\mathbf{X}} H^{1}\left(\Gamma^{*}\right)=\int_{\Gamma^{*}}\left(t^{\prime} \cdot D \mathbf{X} \cdot t\right) d H^{1}
$$

is standard, and

$$
\begin{aligned}
\delta_{\mathbf{X}} \int_{U_{i}}\left(g-a_{i}\right)^{2} d L^{2} & =\int_{U_{i}} \operatorname{div}\left(\left(g-a_{i}\right)^{2} \mathbf{X}\right) d L^{2} \quad \text { by direct computation, } \\
& =-\int_{\partial^{*} U_{i}}\left(g-a_{i}\right)^{2}\left(\eta_{i} \cdot \mathbf{X}\right) d H^{1} \quad \text { by De Giorgi's theorem. }
\end{aligned}
$$

Since $\left\{U_{i}, a_{i}\right\}$ minimizes $E_{n}, \delta_{\mathrm{x}} E_{n}=0$; hence

$$
\begin{aligned}
0 & =\nu_{0} \int_{\Gamma^{*}}\left(t^{\prime} \cdot D \mathbf{X} \cdot t\right) d H^{1}-\sum_{i=1}^{n} \int_{\partial^{*} U_{i}}\left(g-a_{i}\right)^{2}\left(\eta_{i} \cdot \mathbf{X}\right) d H^{1} \\
& =\nu_{0}\left[\int_{\Gamma^{*}}\left(t^{\prime} \cdot D \mathbf{X} \cdot t\right) d H^{1}+\int_{\Gamma^{*}} \kappa \cdot \mathbf{X} d H^{1}\right] .
\end{aligned}
$$

(b) The fact that $\Theta\left(H^{1}, \Gamma^{*}, x\right)$ exists at every point of $R$ and that it is upper semicontinuous follows from the monotonicity formula (see Corollary 17.8 in [13] or Almgren-Allard [1]). Since $\Gamma^{*}$ is rectifiable, $\Theta\left(H^{1}, \Gamma^{*}, x\right)=.1 \quad H^{1}$-a.e. on $\Gamma^{*}$. (This follows from Lemma 5.4.) Hence, $\Theta\left(H^{1}, \Gamma, x\right) \geqq 1$ for all $x \in \Gamma \cap R$ by upper semicontinuity. To extend this argument to $\vec{R}$, we use the reflection technique explained in the beginning of this proof. Then $\Theta\left(H^{1}, \Gamma^{\#, *}, x\right)$ exists, is at least 1 and is upper semi-continuous on $R^{\#}$. But it is easy to see that $\Theta\left(H^{1}, \Gamma^{\#, *}, x\right)=\varepsilon(x) \Theta\left(H^{1}, \Gamma^{*}, x\right)$ for $x \in R^{*} \cap \bar{R}$, i.e., along edges of $R, \Gamma^{*}$ is half of $\Gamma^{\#, *}$, and at corners, $\Gamma^{*}$ is a quarter of $\Gamma^{\#, *}$.

(c) This follows from part (b).

(d) Let $U=R-\Gamma$. Since $L^{2}(\Gamma)=0$, we may replace $U_{i}$ by $U_{i} \cap U$ without altering $E_{n}\left(\left\{U_{i}, a_{i}\right\}\right)$. Let $\left\{V_{\alpha}\right\}$ be the set of components of $U$. Then $\vec{V}_{\alpha}=$ $\Sigma_{i} \overrightarrow{\left(U_{i} \cap V_{\alpha}\right)}$; hence, by the constancy theorem, $\vec{V}_{\alpha}=\overrightarrow{U_{i(\alpha)} \cap V_{\alpha}}$ for exactly one $i(\alpha)$. Therefore we may replace $U_{i}$ by $\cup_{\alpha}\left\{V_{\alpha} \mid i(\alpha)=i\right\}$. 
We now consider the tangent cones of $\Gamma$. Let

$$
\Gamma_{\mathrm{reg}}=\left\{x \in \Gamma: \varepsilon \Theta\left(H^{1}, \Gamma, x\right)=1\right\}
$$

and

$$
\Gamma_{\text {sing }}=\left\{x \in \Gamma: \varepsilon \Theta\left(H^{1}, \Gamma, x\right)>1\right\}
$$

By Theorem 6.3 in [13], for any sequence $\rho_{k} \downarrow 0$, there exists a subsequence $\rho_{k(j)} \downarrow 0$ and Cacciopoli sets $\left\{V_{i}\right\}$ such that, for $1 \leqq i \leqq n,\left(U_{i}\right)_{x, \rho_{k(j)}} \rightarrow V_{i}$ in the $L^{1}\left(\mathbb{R}^{2}\right)$ sense.

Lemma 5.6. Suppose $\left\{U_{i}, a_{i}\right\}$ minimizes $E_{n}$. Let $x \in \Gamma \cap R$. Let $\rho_{k} \downarrow 0$ be a sequence such that, for $1 \leqq i \leqq n,\left(U_{i}\right)_{x, p_{k}} \rightarrow V_{i}$. Let $N^{*}=\cup_{i=1}^{n} \partial^{*} V_{i}$ and let $N=\left(\right.$ closure of $N^{*}$ in $\left.\mathbb{R}^{2}\right)$. Then:

(a) $\Gamma_{x, \rho_{k}} \rightarrow N^{*}$; that is, for all $f \in L^{1}\left(\mathbb{R}^{2}\right)$ with compact support,

$$
\int_{\left(\Gamma_{\left.x, p_{k}\right)}\right.} f d H^{1} \rightarrow \int_{N^{*}} f d H^{1}
$$

(b) $N^{*}$ is stationary in $\mathbb{R}^{2}$; that is, for all $C^{1}$-vector fields $\mathbf{X}$ on $\mathbb{R}^{2}$ with compact support,

$$
\int_{N^{*}}\left(t_{N^{*}}^{\prime} \cdot D \mathbf{X} \cdot t_{N^{*}}\right) d H^{1}=0
$$

Hence, we may assume that the $V_{i}$ are open and $N$ is the union of their topological boundaries.

(c) $N$ is a finite union of rays and each $V_{i}$ is a finite union of sectors of $\mathbb{R}^{2}$.

(d) $\Theta\left(H^{1}, \Gamma, x\right) \in \frac{1}{2} \mathbb{Z}$.

(e) If $\left\{t_{i}\right\}$ is the set of unit tangent vectors along the rays of $N$, pointing away from the origin, then $\sum t_{i}=0$.

Finally, if $x \in \Gamma \cap \partial R$, (a) and (b) hold for the extension $\left\{U_{i}^{\#}, \Gamma^{\#}\right\}$ described above; hence (c) holds, $\varepsilon(x) \Theta\left(H^{1}, \Gamma, x\right) \in \frac{1}{2} \mathbb{Z}$ and, along edges of $R, \sum t_{i}$ is normal to the edge.

Proof: To simplify notation in this proof, let

$$
f_{k}(y)=\rho_{k}^{-1}(y-x)
$$

so that, for any set $S, S_{x, \rho_{k}}$ is the same as $f_{k}(S)$ : the set $S$ expanded around $x$ by a large factor $\rho_{k}^{-1}$.

To prove (a), it is enough to show that

(i) for all $W \subset \mathbb{R}^{2}$ open, $H^{1}\left(N^{*} \cap W\right) \leqq \liminf _{k} H^{1}\left(f_{k}(\Gamma) \cap W\right)$;

(ii) for all $K \subset \mathbb{R}^{2}$ compact, $H^{1}\left(N^{*} \cap K\right) \geqq \lim \sup _{k} H^{1}\left(f_{k}(\Gamma) \cap K\right)$. 
Since $\partial \overrightarrow{f_{k}\left(U_{i}\right)} \rightarrow \partial \vec{V}_{i}, \mathbf{M}_{W}\left(\partial \vec{V}_{i}\right) \leqq \liminf \mathbf{M}_{W}\left(\overrightarrow{f_{k}\left(U_{i}\right)}\right)$ for all open $W$. Hence (i) follows. Now fix $K \subset \mathbb{R}^{2}$ compact and $\varepsilon>0$. Choose a smooth function $\varphi: \mathbb{R}^{2} \rightarrow[0,1]$ such that

$$
\begin{aligned}
& \varphi \equiv 1 \text { on } K \\
& \operatorname{supp}(\varphi) \subset\{y \mid \operatorname{dist}(y, K)<\varepsilon\}
\end{aligned}
$$

Let $k_{0}$ be an integer such that

$$
\operatorname{supp}(\varphi) \subset f_{k}(R) \text { for } k \geqq k_{0}
$$

Let

$$
\begin{aligned}
W_{\alpha} & =\{y \mid \varphi(y)>\alpha\} \\
\vec{P}_{i}^{(k)} & =\vec{V}_{i}-f_{k}\left(\vec{U}_{i}\right) \quad \text { (difference of 2-currents). }
\end{aligned}
$$

Then $\mathbf{M}_{W}\left(\vec{P}_{i}^{(k)}\right) \rightarrow 0$ for all open $W \subset \mathbb{R}^{2}$. By the slicing theorem, we may choose $0<\alpha<1$ such that for $1 \leqq k \leqq n$ and $k \geqq k_{0}$ :

$$
\begin{aligned}
\partial\left(\vec{P}_{i}^{(k)}\left\llcorner W_{\alpha}\right)\right. & =\left(\partial \vec{P}_{i}^{(k)}\right)\left\llcorner W_{\alpha}+\vec{Q}_{i}^{(k)},\right. \\
\vec{Q}_{i}^{(k)} & =\text { "slice" of } \vec{P}_{i}^{(k)} \text { by } \partial W_{\alpha},
\end{aligned}
$$

so that

$$
\begin{gathered}
\operatorname{supp}\left(\vec{Q}_{i}^{(k)}\right) \subset \partial W_{\alpha}, \\
\lim _{k} \mathbf{M}\left(\vec{Q}_{i}^{(k)}\right)=0 .
\end{gathered}
$$

Moreover, for suitable $\alpha$, we may assume

$$
\mathbf{M}\left(\overrightarrow{\partial f_{k}\left(U_{i}\right)}\left\llcorner\partial W_{\alpha}\right)=\mathbf{M}\left(\partial \vec{V}_{i}\left\llcorner\partial W_{\alpha}\right)=0\right.\right.
$$

The main idea is to define, for all $k$, a modified decomposition of $R$ into Cacciopoli sets $\left\{U_{i}^{(k)}\right\}$, namely:

$$
\begin{aligned}
U_{i}^{(k)} \cap f_{k}^{-1}\left(W_{\alpha}\right) & =f_{k}^{-1}\left(V_{i}\right), \\
U_{i}^{(k)} \cap\left(R-f_{k}^{-1}\left(W_{\alpha}\right)\right) & =U_{i},
\end{aligned}
$$

or alternately, define $U_{i}^{(k)}$ as a current by

$$
\vec{U}_{i}^{(k)}=\vec{U}_{i}+f_{k}^{-1}\left(\vec{P}_{i}^{(k)}\left\llcorner W_{\alpha}\right)\right.
$$



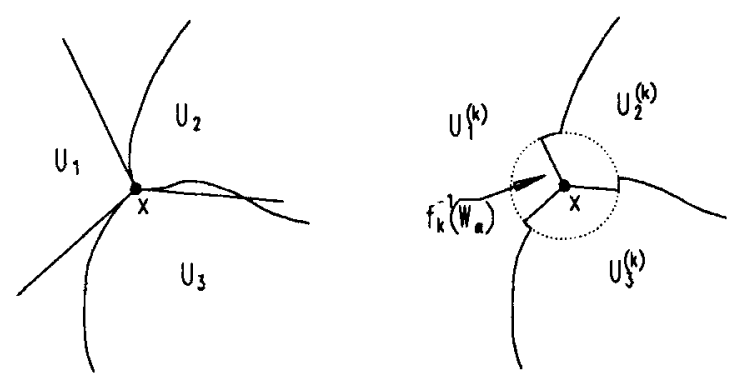

Figure 21. Modifying a decomposition via its tangent cone.

(see Figure 21). It is easy to check, using the second description, that

$$
\mathbf{M}\left(\partial \vec{U}_{i}^{(k)}\right)=\mathbf{M}\left(\partial \vec{U}_{i}\right)+\rho_{k}\left[\mathbf{M}_{W_{a}}\left(\partial \vec{V}_{i}\right)-\mathbf{M}_{W_{\mathrm{a}}}\left(\partial f_{k}\left(\vec{U}_{i}\right)\right)+\mathbf{M}\left(\vec{Q}_{i}^{(k)}\right)\right]
$$

Letting $\omega_{i, k}(y)=\left(g\left(f_{k}^{-1}(y)\right)-\alpha_{i}\right)^{2} d x d y$, we find

$$
\begin{aligned}
E_{n}\left(\left\{U_{i}^{(k)}, a_{i}\right\}\right)= & E_{n}\left(\left\{U_{i}, a_{i}\right\}\right) \\
& +\frac{1}{2} \nu_{0} \rho_{k} \sum_{i=1}^{n}\left[\mathbf{M}_{W_{\alpha}}\left(\partial \vec{V}_{i}\right)-\mathbf{M}_{W_{\alpha}}\left(\partial \overrightarrow{f_{k}\left(U_{i}\right)}\right)+\mathbf{M}\left(\vec{Q}_{i}^{(k)}\right)\right] \\
& +\rho_{k}^{2} \sum_{i=1}^{n}\left(\vec{P}_{i}^{(k)}\left\llcorner W_{\alpha}\right)\left(\omega_{i, k}\right) .\right.
\end{aligned}
$$

But now $E_{N}\left(\left\{U_{i}^{(k)}, a_{i}\right\}\right) \geqq E_{n}\left(\left\{U_{i}, a_{i}\right\}\right)$ by hypothesis, so

$$
\begin{aligned}
0 \leqq & H^{1}\left(N^{*} \cap W_{\alpha}\right)-H^{1}\left(f_{k}(\Gamma) \cap W_{\alpha}\right) \\
& +\sum_{i=1}^{n}\left[\frac{1}{2} \mathbf{M}\left(\vec{Q}_{i}^{(k)}\right)+\frac{\rho_{k}}{\nu_{0}}\left(\vec{P}_{i}^{(k)}\left\llcorner W_{\alpha}\right)\left(\omega_{i, k}\right)\right] .\right.
\end{aligned}
$$

It follows that

$$
H^{1}\left(N^{*} \cap W_{\alpha}\right) \geqq \lim \sup H^{1}\left(f_{k}(\Gamma) \cap W_{\alpha}\right)
$$

and hence

$$
H^{1}\left(N^{*} \cap\{y: \operatorname{dist}(y, K)<\varepsilon\}\right) \geqq \limsup H^{1}\left(f_{k}(\Gamma) \cap K\right) .
$$

Letting $\varepsilon \downarrow 0$, we get

$$
H^{1}\left(N^{*} \cap K\right) \geqq \lim \sup H^{1}\left(f_{k}(\Gamma) \cap K\right) .
$$

It follows that $f_{k}(\Gamma) \rightarrow N^{*}$. 
To prove (b), note that $f_{k}(\Gamma)$ has generalized curvature $\kappa_{k}$ such that $\left|\kappa_{k}(y)\right|$ $=\rho_{k}\left|\kappa\left(\rho_{k} y+x\right)\right|$. Therefore, $N$ is stationary by lower semicontinuity of variation measures (Theorem 40.6 in [13]). The rest of part (b) now follows as in Lemma 5.5. Part (c) follows from Theorem 19.3 in [13]. (d) holds because $\Theta\left(H^{1}, \Gamma, x\right)=\Theta\left(H^{1}, N,\{0\}\right)$. Part (e) follows from part (b). The extension to $\partial R$ follows immediately by reflection.

LEMMA 5.7. If $\left\{U_{i}, a_{i}\right\}$ minimizes $E_{n}$, then $\Gamma_{\text {sing }}$ is finite.

Proof: Suppose that $\Gamma_{\text {sing }}$ is not finite. Let $x_{1}, x_{2}, \cdots$ be a sequence of points in $\Gamma_{\text {sing }}-\{x\}$ converging to $x$. If $x \in \partial R$, replace $\left\{U_{i}, a_{i}\right\}$ by $\left\{U_{i}^{*}, a_{i}\right\}$. Let $\rho_{k}=\left|x_{k}-x\right|$. If we replace $\left\{\rho_{k}\right\}$ by a suitable subsequence, then $\Gamma_{x, \rho_{k}}$ converges to a cone $N$ as in Lemma 5.6. The points $\xi_{k}=\rho_{k}^{-1}\left(x_{k}-x\right)$ are on $\partial B_{1}(0)$ and hence converge to a point $\xi \in \partial B_{1}(0)$. By monotonicity and Lemma $5.6, \Theta\left(H^{1}, N, \xi\right) \geqq \lim \sup \Theta\left(H^{1}, \Gamma_{x, \rho_{k}}, \xi_{k}\right) \geqq 1 \frac{1}{2}$. Therefore, $\xi \in N-\{0\}$. But, $\Theta\left(H^{1}, N, y\right)=1$ for all $y \in N-\{0\}$ by Lemma 5.6. Contradiction!

LeMmA 5.8. If $\left\{U_{i}, a_{i}\right\}$ minimizes $E_{n}$, then $\Gamma_{\mathrm{reg}}$ is $C^{2}$.

Proof: By Allard's regularly theorem (see Theorem 24.2 in [13]), $\Gamma_{\text {reg }}$ is $C^{1}$. Its curvature $\kappa$ is $C^{0}$ along $\Gamma_{\text {reg }}$ since $g$ is. It follows from the standard regularity theory of elliptic differential equations that $\Gamma_{\text {reg }}$ must be $C^{2}$.

Proof of Theorem 5.2: Parts (a) and (b) have already been proven. Note that the theory of Section 1 now applies and we have a classification of the singularities of $\Gamma$. The key point in proving (c) is to show that

$$
|R \cap \partial W| \geqq \min \left\{\frac{\pi \nu_{0}}{12|g|_{\max }^{2}}, \quad \text { width }(R)\right\}
$$

for all components $W$ of $R-\Gamma$. Notice that the inequality does not depend on $n$. To prove the inequality, suppose that there is a $W$ for which the inequality is false. First consider the case when one of the components $\gamma$ of $\partial W$ is wholly contained in $R$, i.e., does not meet $\partial R$. Let $m$ be the number of singular points on $\gamma$. We claim that $m \leqq 6$. To see this, orient $\gamma$ and let $\left\{\theta_{i}\right\}$ be the set of angle changes in the tangent directions at the singular points of $\gamma$ (see Figure 22).

By the Gauss-Bonnet formula,

$$
2 \pi=\sum \theta_{i}+\int_{\gamma} \kappa \cdot n
$$

where $\kappa$ is the curvature vector and $n$ is the inward unit normal. By the results of 


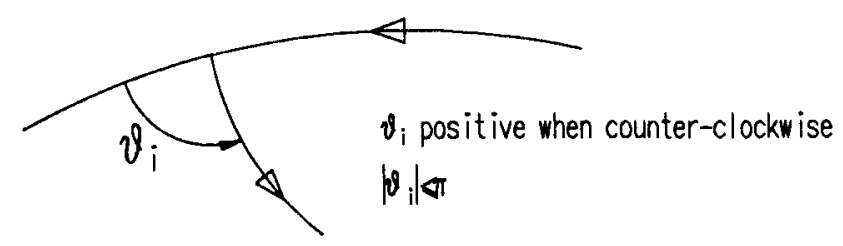

Figure 22. Conventions for the exterior angle $\theta_{i}$.

Section $1, \theta_{i}=\frac{1}{3} \pi$ for all $i$. By Lemmas 5.4 and 5.5,

$$
|\kappa| \leqq \frac{4|g|_{\max }^{2}}{\nu_{0}}
$$

It follows that

$$
m \leqq 6+\frac{12}{\pi \nu_{0}}|g|_{\max }^{2}|\gamma|<7
$$

Therefore $\gamma$ contains a $C^{2}$-arc $l$ of length greater than or equal to $\frac{1}{6}|\gamma|$. Suppose $l \subset U_{i} \cap U_{j}$, where $U_{i}$ meets $l$ along the side interior to $\gamma$. Let $W^{\prime}$ be the component of $U_{i}$ meeting $l$. Consider a new segmentation $\left\{U_{i}^{\prime}\right\}$ of $R$ so that

$$
\begin{gathered}
\vec{U}_{i}^{\prime}=\vec{U}_{i}-\vec{W}^{\prime}, \\
\vec{U}_{j}^{\prime}=\vec{U}_{j}+\vec{W}^{\prime}, \\
U_{k}^{\prime}=U_{k} \text { if } k \neq i, j . \\
\Delta E_{n}=E_{n}\left(\left\{U_{i}, a_{i}\right\}\right)-E_{n}\left(\left\{U_{i}^{\prime}, a_{i}\right\}\right) \geqq \nu_{0} l-2|g|_{\max }^{2} \cdot \operatorname{area}\left(W^{\prime}\right) .
\end{gathered}
$$

By the isoperimetric inequality,

$$
\operatorname{area}\left(W^{\prime}\right) \leqq \frac{|\gamma|^{2}}{4 \pi}
$$

Therefore,

$$
\begin{aligned}
\Delta E_{n} & \geqq \frac{1}{6} \nu_{0}|\gamma|-\frac{|g|_{\max }^{2}}{2 \pi}|\gamma|^{2} \\
& \geqq \frac{1}{6} \nu_{0}|\gamma|\left[1-\frac{3|g|_{\max }^{2}}{\pi \nu_{0}}|g|\right] \\
& >\frac{1}{6} \nu_{0}|\gamma|\left(\frac{3}{4}\right)>0
\end{aligned}
$$

which is a contradiction since $\left\{U_{i}, a_{i}\right\}$ minimizes $E_{n}$. 
If $R \cap \partial W$ does not contain a loop, then it must have a component $\gamma$ meeting $\partial R$ twice. Moreover, since $|R \cap \partial W|<$ width $(R), \gamma$ cannot meet the opposite sides of $R$. Now we apply the reflection construction where $R^{\#}$ is obtained by reflecting $R$ across the two sides met by $\gamma$. Let $W^{\#}$ be the set in $R^{\#}$ corresponding to $W$, obtained by reflecting $W$. Then $\partial W^{*}$ must contain a loop $\gamma^{\#}$. We argue now as before to get a contradiction again.

Observe that

$$
\begin{aligned}
E_{n}\left(\left\{U_{i}, a_{i}\right\}\right) & =\frac{1}{2} \nu_{0} \sum_{\substack{\text { components } \\
W \text { of } R-\Gamma}}|R \cap \partial W|+\sum_{i=1}^{n} \int_{U_{i}}\left(g-a_{i}\right)^{2} \\
& \leqq E_{n}\left(\left\{V_{i}, b_{i}\right\}\right), \text { where } V_{1}=R, b_{1}=0 \text { and } V_{i}=\varnothing \text { for } i>1, \\
& \leqq|g|_{\text {max }}^{2} \cdot \operatorname{area}(R) .
\end{aligned}
$$

Therefore,

$$
\text { [number of components of } R-\Gamma] \leqq \frac{\left(2 / \nu_{0}\right)|g|_{\max }^{2} \cdot \operatorname{area}(R)}{\min \left\{\pi \nu / 12|g|_{\max }^{2}, \operatorname{width}(R)\right\}}
$$

Part (c) of Theorem 5.2 now follows.

\section{Approximation when $\mu$ is Large}

We derive here limiting forms of the energy and its first variation (along smooth portions of $\Gamma$ ) as $\mu \rightarrow \infty$. When $\mu$ is large, the effect of $\Gamma$ on the energy is essentially confined to a narrow strip along $\Gamma$. We can even express the contribution of $\Gamma$ to the energy as an integral along $\Gamma$ and analyze the first variation in the form of variation of this line integral. The whole approach is based on the following:

LEMMA. Suppose $g_{\mu}$ satisfies the equation $\nabla^{2} g_{\mu}=\mu^{2}\left(g_{\mu}-g\right)$ everywhere in $R$ and $\partial g_{\mu} /\left.\partial n\right|_{\partial R}=0$. Let $f_{\Gamma}$ minimize $E(f, \Gamma)$ with $\Gamma$ fixed. Let $h_{\Gamma}=f_{\Gamma}-g_{\mu}$. Then

$$
E\left(f_{\Gamma}, \Gamma\right)=E\left(g_{\mu}, \varnothing\right)-\int_{\Gamma}\left[\frac{\partial g_{\mu}}{\partial n}\left(h_{\Gamma}^{+}-h_{\Gamma}^{-}\right)-\nu\right] d s,
$$

where the superscripts + and - distinguish between the values of a variable on the two sides of $\Gamma$ and $\partial / \partial n$ is the normal derivative in the direction from the - side to the + side. 
Proof:

$$
\begin{aligned}
E\left(f_{\Gamma}, \Gamma\right)-E\left(g_{\mu}, \phi\right) & \\
= & \iint_{R-\Gamma}\left[\mu^{2}\left(f_{\Gamma}-g\right)^{2}+\left\|\nabla f_{\Gamma}\right\|^{2}\right]+\nu|\Gamma| \\
& -\iint_{R}\left[\mu^{2}\left(g_{\mu}-g\right)^{2}+\left\|\nabla g_{\mu}\right\|^{2}\right] \\
= & \iint_{R-\Gamma}\left[2\left\{\mu^{2}\left(g_{\mu}-g\right) h_{\Gamma}+\nabla g_{\mu} \cdot \nabla h_{\Gamma}\right\}\right. \\
& \left.\quad+\left\{\mu^{2} h_{\Gamma}^{2}+\left\|\nabla h_{\Gamma}\right\|^{2}\right\}\right]+\nu|\Gamma| \\
= & \iint_{R-\Gamma}\left[2 h_{\Gamma}\left\{\mu^{2}\left(g_{\mu}-g\right)-\nabla^{2} g_{\mu}\right\}+h_{\Gamma}\left(\mu^{2} h_{\Gamma}-\nabla^{2} h_{\Gamma}\right)\right] \\
& -\int_{\Gamma}\left[2 \frac{\partial g_{\mu}}{\partial n}\left(h_{\Gamma}^{+}-h_{\Gamma}^{-}\right)+\frac{\partial h_{\Gamma}^{+}}{\partial n} h_{\Gamma}^{+}-\frac{\partial h_{\Gamma}^{-}}{\partial n} h_{\Gamma}^{-}-\nu\right] d s,
\end{aligned}
$$

by Green's identity.

The lemma follows since the first integrand in the last step is zero and $\partial h_{\Gamma}^{+} / \partial n$ $=\partial h_{\Gamma} / \partial n=-\partial g_{\mu} / \partial n$ along $\Gamma$.

Thus $\Gamma$ minimizes $E\left(f_{\Gamma}, \Gamma\right)$ if and only if it maximizes the line integral

$$
\int_{\Gamma}\left[\frac{\partial g_{\mu}}{\partial n}\left(h^{+}-h^{-}\right)-\nu\right] d s,
$$

where $h$ is the solution to the boundary value problem

$$
\begin{aligned}
\nabla^{2} h & =\mu^{2} h \quad \text { on } \quad R-\Gamma, \\
\frac{\partial h}{\partial n} & =-\frac{\partial g_{\mu}}{\partial n} \quad \text { along } \quad \Gamma \cup \partial R .
\end{aligned}
$$

To understand the limiting behavior of this integral as $\mu \rightarrow \infty$, we need to describe the asymptotic behavior of $h_{\Gamma}$ as $\mu \rightarrow \infty$. This involves considerable technical details, which we have put in Appendices 2 and 3. Appendix 2 is devoted to proving that

$$
\sup _{P \in R}\left|h_{\Gamma}(P)\right|=O(1 / \mu) \text { as } \mu \rightarrow \infty
$$

This estimate is very simple away from $\Gamma$ and near smooth points of $\Gamma$, but to prove this near singularities of $\Gamma$ seems harder (in fact, we had to exclude cusps on $\Gamma$ ). Appendix 3 is devoted to studying $h_{\Gamma}$ near smooth points of $\Gamma$ and 
deriving the precise asymptotic form. Introduce coordinates $r$ and $s$ along $\Gamma$, where $r(P)$ is the distance from $P$ to the nearest point $\bar{P}$ on $\Gamma$ and $s(P)$ is arc length on $\Gamma$ from some origin to $\bar{P}$ (see Figure 3, Section 1). We can prove that if $\Gamma$ is sufficiently smooth, e.g. $C^{3,1}$, then

$$
h_{\Gamma}(r, s)=\frac{e^{-\mu r}}{\mu \sqrt{1-\kappa(s) r}}\left(1+\frac{\kappa(s)}{2 \mu}\right) \frac{\partial g}{\partial n}(0, s)+O\left(\frac{1}{\mu^{3}}\right),
$$

where $\kappa(s)$ is the curvature of $\Gamma$ at $s$. The proof is rather long, however, but the essence of it, a careful application of Green's theorem, is given in Appendix 3 for the points on $\Gamma$, where we shall use it. It is easy to derive the form of this expansion for $h_{\Gamma}$ by examining the case $\Gamma=$ circle, and using the explicit expression for $h_{\Gamma}$ in terms of Bessel functions of the 2-nd kind (cf. [14], Ch. 17).

An interesting question is to find the asymptotic expansion of $h_{\Gamma}$ for large $\mu$ near the singularities of $\Gamma$. We were quite puzzled looking for appropriate "elementary" functions from which to construct this expansion. In the case where $\Gamma$ is the positive $x$-axis, John Myers found a beautiful explicit formula for the $h_{\Gamma}$ satisfying

(a) $\nabla^{2} h_{\Gamma}=\mu^{2} h_{\Gamma}$,

(b) $\partial h_{\Gamma} / \partial n=1$ along $\Gamma$,

(c) $h_{\Gamma}(x, y) \approx \operatorname{sgn}(y) \cdot e^{-\mu y} / \mu$ if $x \gg 0,|y| \leqq C$,

(d) $h_{\Gamma}=O\left(e^{-\mu r}\right)$ if $0<\theta_{0} \leqq \theta \leqq 2 \pi-\theta_{0}$.

Using the error function, he introduces

$$
g(x, y)=\frac{e^{-\mu y}}{\mu \sqrt{\pi}} \int_{-\infty}^{\mathscr{R} e(1+i) \sqrt{\mu z}} \exp \left\{-s^{2}\right\} d s .
$$

One can check that (a) and (d) hold for $g$ and that (b) and (c) also hold for

$$
h_{\Gamma}(x, y)=g(x, y)-g(x,-y) \text {. }
$$

Using this special $h_{\Gamma}$, one should be able to construct good asymptotic approximations to the general $h_{\Gamma}$ near crack-tips, as $\mu \rightarrow \infty$.

Applying the estimates for $h_{\Gamma}$ in Appendices 2 and 3, we can estimate the behavior of $E\left(f_{\Gamma}, \Gamma\right)$ when $\mu \rightarrow \infty$ and $\Gamma$ is fixed. As in the introduction, we write

$$
E_{\infty}(\Gamma)=\int_{\Gamma}\left[\nu_{\infty}-\left(\frac{\partial g}{\partial n}\right)^{2}\right] d s
$$

We now set $\nu_{\infty}=\frac{1}{2} \mu \nu$. Then we have

THEOREM 6.1. Suppose that $g$ is $C^{1,1}$ and that $\Gamma$ is the union of finitely many $C^{1,1}$-arcs. Assume that $\Gamma \cup \partial R$ has no cusps. Then, as $\mu \rightarrow \infty$,

$$
E\left(f_{\Gamma}, \Gamma\right)=E\left(g_{\mu}, \varnothing\right)+\frac{2}{\mu} E_{\infty}(\Gamma)+\|g\|_{2, \infty, R}^{2} O\left(\frac{\log \mu}{\mu^{2}}\right) .
$$


Proof: We first construct $g_{\mu}$ and derive estimates for it. Pave $\mathbb{R}^{2}$ by successive reflections of $R$ about edges and thus extend $g$ to all of $\mathbb{R}^{2}$. The solution $g_{\mu}$ can then be expressed as

$$
g_{\mu}(P)=\frac{\mu^{2}}{2 \pi} \iint_{\mathbb{R}_{Q}^{2}} K_{0}(\mu|P Q|) g(Q)
$$

for all $P \in \mathbb{R}^{2}$. Here $K_{0}$ is the zeroth-order modified Bessel function of the second kind.

We need estimates on $g_{\mu}$ and its first and second derivatives:

LemMa. For all points $P$ in $R$ and all $\mu$ such that $\mu \cdot \operatorname{dist}(P, \partial R) \geqq$ $3 \log (\mu|\partial R|)$

$$
\begin{gathered}
g_{\mu}(P)=g(P)+\|g\|_{2, \infty, R} O\left(\frac{1}{\mu^{2}}\right), \\
\nabla g_{\mu}(P)=\nabla g(P)+\|g\|_{2, \infty, R} O\left(\frac{1}{\mu}\right), \\
\left|\frac{\partial^{2} g_{\mu}}{\partial x_{i} \partial x_{j}}\right|_{P} \leqq \text { const. }\|g\|_{2, \infty, R} \quad \text { for } \quad 1 \leqq i, j \leqq 2 .
\end{gathered}
$$

Moreover,

$$
\left\|g_{\mu}\right\|_{1, \infty, R} \leqq \text { const. }\|g\|_{0, \infty, R}
$$

Proof: To see this, we first note that, for $n \geqq 0$ and $i=0$ or 1 ,

$$
\iint_{\mathbb{R}^{2}} r^{n} K_{i}(\mu r)=2 \pi \int_{0}^{\infty} r^{n+1} K_{i}(\mu r) d r=\frac{2 \pi}{\mu^{n+2}} \int_{0}^{\infty} z^{n+1} K_{i}(z) d z=O\left(\frac{1}{\mu^{n+2}}\right)
$$

and

$$
\begin{aligned}
\iint_{r \geqq d} K_{i}(\mu r)=2 \pi \int_{d}^{\infty} r K_{i}(\mu r) d r & =\frac{2 \pi}{i^{2}} \int_{\mu d}^{\infty} z K_{i}(z) d z \\
& \leqq(\mu d)^{1 / 2} e^{-\mu d} O\left(\frac{1}{\mu^{2}}\right)
\end{aligned}
$$

by Lemma 3 in Appendix 3,

$$
\leqq O\left(\frac{1}{\mu^{4}}\right) \quad \text { if } \quad \mu d \geqq 3 \log (\mu|\partial R|) .
$$


Let $d=\operatorname{dist}(P, \partial R)$ and let $U_{d}$ be the disc of radius $d$ with center at $P$. Since

$$
\begin{gathered}
\frac{\mu^{2}}{2 \pi} \iint_{\mathbb{R}^{2}} K_{0}(\mu r)=1, \\
g_{\mu}(P)-g(P)=\frac{\mu^{2}}{2 \pi} \iint_{\mathbb{R}_{Q}^{2}} K_{0}(\mu r)[g(Q)-g(P)]
\end{gathered}
$$

we find

$$
\begin{aligned}
& \left|\iint_{U_{d}} K_{0}(\mu r)[g(Q)-g(P)]\right| \\
& \quad \leqq\left|\iint_{U_{d}} K_{0}(\mu r)\left[\left.\frac{\partial g}{\partial x}\right|_{P} x+\left.\frac{\partial g}{\partial y}\right|_{P} y+\|g\|_{2, \infty, R} O\left(r^{2}\right)\right]\right| \\
& \quad \leqq\|g\|_{2, \infty, R} O\left(\frac{1}{\mu^{4}}\right)
\end{aligned}
$$

and

$$
\left|\iint_{\mathbb{R}_{Q}^{2}-U_{d}} K_{0}(\mu r)[g(Q)-g(P)]\right| \leqq\|g\|_{0, \infty, R} O\left(\frac{1}{\mu^{4}}\right)
$$

Likewise,

$$
\begin{aligned}
\nabla g_{\mu}(P)-\nabla g(P) & =\frac{\mu^{2}}{2 \pi} \iint_{\mathbf{R}_{Q}^{2}}\left[\nabla_{P} K_{0}(\mu r) g(Q)-K_{0}(\mu r) \nabla g(P)\right] \\
& =\frac{\mu^{2}}{2 \pi} \iint_{\mathbf{R}_{Q}^{2}}\left[-\nabla_{Q} K_{0}(\mu r) g(Q)-K_{0}(\mu r) \nabla g(P)\right], \\
\mid \int_{U_{d}}\left[\nabla_{Q} K_{0}(\mu r) g(Q)\right. & \left.+K_{0}(\mu r) \nabla g(Q)\right] \mid \leqq 2 \pi K_{0}(\mu d)\|g\|_{0, \infty, R},
\end{aligned}
$$

by Green's identity,

$$
\leqq \text { const. }(\mu d)^{-1 / 2} e^{-\mu d}\|g\|_{0, \infty, R}
$$

by Lemma 3, Appendix 3,

$$
\leqq\|g\|_{0, \infty, R} O\left(\frac{1}{\mu^{3}}\right) \text {. }
$$


Therefore,

$$
\begin{aligned}
& \left|\iint_{U_{d}} \nabla_{Q} K_{0}(\mu r) g(Q)+K_{0}(\mu r) \nabla g(P)\right| \\
& \quad \leqq\left|\iint_{U_{d}} K_{0}(\mu r)[\nabla g(Q)-\nabla g(P)]\right|+\|g\|_{0, \infty, R} O\left(\frac{1}{\mu^{3}}\right) \\
& \quad \leqq\left|\iint_{U_{d}} K_{0}(\mu r) O(r)\|g\|_{2, \infty, R}\right|+\|g\|_{0, \infty, R} O\left(\frac{1}{\mu^{3}}\right) \\
& \quad \leqq\|g\|_{2, \infty, R} O\left(\frac{1}{\mu^{3}}\right) .
\end{aligned}
$$

Also

$$
\begin{aligned}
& \left|\iint_{\mathbf{R}_{Q}^{2}-U_{d}}\left[\nabla_{Q} K_{0}(\mu r) g(Q)-K_{0}(\mu r) \nabla g(P)\right]\right| \\
& \quad \leqq \iint_{\mathbb{R}_{Q}^{2}-U_{d}}\left[\mu K_{1}(\mu r)|g(Q)|+K_{0}(\mu r) \mid \nabla g(P) \|\right] \\
& \quad \leqq\|g\|_{1, \infty, R} O\left(\frac{1}{\mu^{3}}\right) .
\end{aligned}
$$

The remaining estimates may be proved in the same way.

We proceed now with the proof of the theorem. If $\Gamma$ contains arcs which terminate in free ends (i.e., crack-tips), extend these arcs in some $C^{1,1}$ way until they meet $\Gamma \cup \partial R$ (without creating cusps, however). Let $\bar{\Gamma}$ denote the extended $\Gamma$. In order to apply the theorem in Appendix 3, we need to define several constants which depend only on $\Gamma$. If $\gamma \subset \gamma^{*} \subset \Gamma$ are curves, let

$$
d\left(\gamma, \gamma^{*}\right)=\left\{\begin{array}{c}
\infty, \text { if } \gamma^{*} \text { is a connected closed curve; } \\
\text { arc length between } \gamma \text { and the end points of } \gamma^{*}, \\
\text { if } \gamma \text { and } \gamma^{*} \text { are connected and open; } \\
\min _{i} d\left(\gamma_{i}, \gamma_{i}^{*}\right), \text { in general, where } \gamma_{i} \text { are the components of } \gamma \text { and } \\
\gamma_{i}^{*} \text { is the component of } \gamma^{*} \text { containing } \gamma_{i} .
\end{array}\right.
$$


For some $\varepsilon>0$, we introduce constants:

$$
\begin{aligned}
\Gamma_{\text {sing }}= & \text { singular points of } \Gamma \cup \partial R \text { (including the crack-tips), } \\
\Lambda_{P}= & \text { the diameter of the largest circle through } P \\
& \text { contained in a single component of } R-\bar{\Gamma}, \\
\gamma_{\varepsilon}= & \left\{P \in \Gamma \mid \operatorname{dist}\left(P, \Gamma_{\text {sing }}\right) \geqq \varepsilon\right\}, \\
\Lambda_{\varepsilon}= & \min _{P \in \gamma_{\varepsilon}} \Lambda_{P} .
\end{aligned}
$$

Since there are no cusps and since each arc of $\Gamma$ is $C^{1,1}$, there exist constants $C_{1}$, $C_{2}$ and $C_{3}$ such that

$$
\begin{array}{r}
\Lambda_{\varepsilon} \geqq C_{1} \varepsilon, \\
\operatorname{dist}\left(\gamma_{\varepsilon}, \partial R\right) \geqq C_{2} \varepsilon,
\end{array}
$$

and

$$
\operatorname{arc~length}\left(\Gamma-\gamma_{\varepsilon}\right) \leqq C_{3} \varepsilon .
$$

Let $C=\min \left\{\frac{1}{32}, \frac{1}{8} C_{1}, \frac{1}{2} C_{2}\right\}, \alpha_{i}$ be the $C^{2}$-norm of the $i$-th arc of $\Gamma$ and $\alpha=\max _{i} \alpha_{i}$. Let $\beta$ be the universal constant as defined in Lemma 2 of Appendix 3. Let

$$
\mu_{0}=\frac{6 \alpha}{\beta} \log (\mu|\partial R|)
$$

Now fix $\mu \geqq \mu_{0}$ and let $\varepsilon=(6 / \mu C) \log (\mu|\partial R|)$. Let $\gamma=\gamma_{\varepsilon}$ and $\gamma^{*}=\gamma_{\varepsilon / 2}$. Then $d\left(\gamma, \gamma^{*}\right) \geqq \frac{1}{2} \varepsilon$ and if

$$
\delta=\min \left\{\frac{\beta}{\alpha}, \frac{1}{16} d\left(\gamma, \gamma^{*}\right), \frac{1}{4} \Lambda_{\varepsilon / 2}\right\}
$$

then

$$
\mu \delta \geqq 6 \log (\mu|\partial R|)
$$

Moreover,

$$
\mu \operatorname{dist}\left(\gamma^{*}, \partial R\right) \geqq 6 \log (\mu|\partial R|)
$$

and

$$
\text { arc length }(\Gamma-\gamma) \leqq \frac{6 C_{3}}{C} \frac{\log (\mu|\partial R|)}{\mu}
$$


Let $h_{\Gamma}=f_{\Gamma}-g_{\mu}$ as above. We now apply Part A of the theorem in Appendix

3. This shows that, for each component $\Omega$ of $R-\bar{\Gamma}$,

$\left.h_{\Gamma}\right|_{\gamma}=\frac{1}{\mu} \frac{\partial g_{\mu}}{\partial n}+\left\|\frac{\partial g_{\mu}}{\partial n}\right\|_{1, \infty, \gamma^{*}} O\left(\frac{1}{\mu^{2}}\right)+\left\|h_{\Gamma}\right\|_{0, \infty, \partial \Omega} O\left(\frac{1}{\mu^{4}}\right)+\left\|\frac{\partial h_{\Gamma}}{\partial n}\right\|_{0,1, \partial \Omega} O\left(\frac{1}{\mu^{6}}\right)$.

Substitute the following estimates in this formula:

$$
\begin{gathered}
\frac{\partial g_{\mu}}{\partial n}=\frac{\partial g}{\partial n}+\|g\|_{2, \infty, R} O\left(\frac{1}{\mu}\right) \\
\left\|h_{\Gamma}\right\|_{0, \infty, \partial \Omega} \leqq\left\|g_{\mu}\right\|_{0, \infty, \partial \Omega}+\left\|f_{\Gamma}\right\|_{0, \infty, \partial \Omega} \leqq 2\|g\|_{0, \infty, R}
\end{gathered}
$$

Note that $\partial h_{\Gamma} / \partial n$ must be estimated on $\bar{\Gamma}-\Gamma$ as well as on $\Gamma$ and it will certainly have singularities at crack-tips. To deal with the last term, recall that $h_{\Gamma} \in W_{p}^{2}(R-\Gamma)$ for $1 \leqq p<\frac{4}{3}$ by the results in Appendix 1 . Therefore, by Theorem 2.3.3.6 in Grisvard [7] (applied to $g_{\mu}$ and $f_{\Gamma}$ separately),

$$
\left\|h_{\Gamma}\right\|_{2, p, R-\Gamma} \leqq\|g\|_{0, p, R-\Gamma} O\left(\mu^{2}\right) \leqq\|g\|_{0, \infty, R} O\left(\mu^{2}\right)
$$

hence

$$
\left\|\frac{\partial h_{\Gamma}}{\partial n}\right\|_{0,1, a \Omega} \leqq\|g\|_{0, \infty, R} O\left(\mu^{2}\right)
$$

by the trace formula in Sobolev spaces (see [7], Section 1.5). We get

$$
\left.h_{\Gamma}\right|_{\gamma}=\frac{1}{\mu} \frac{\partial g}{\partial n}+\|g\|_{2, \infty, R} O\left(\frac{1}{\mu^{2}}\right)
$$

and

$$
\int_{\gamma} \frac{\partial g_{\mu}}{\partial n}\left(h_{\Gamma}^{+}-h_{\Gamma}^{-}\right) d s=\frac{2}{\mu} \int_{\gamma}\left(\frac{\partial g}{\partial n}\right)^{2} d s+\|g\|_{2, \infty, R}^{2} O\left(\frac{1}{\mu^{2}}\right) .
$$

By the theorem in Appendix 2,

$$
\left\|g_{\mu}-g\right\|_{0, \infty, R} \leqq\|g\|_{1, \infty, R} O\left(\frac{1}{\mu}\right)
$$

and

$$
\left\|f_{\Gamma}-g\right\|_{0, \infty, R} \leqq\|g\|_{1, \infty, R} O\left(\frac{1}{\mu}\right)
$$

Therefore,

$$
\begin{aligned}
\left\|h_{\Gamma}\right\|_{0, \infty, R} & =\left\|f_{\Gamma}-g_{\mu}\right\|_{0, \infty, R} \leqq\left\|f_{\Gamma}-g\right\|_{0, \infty, R}+\left\|g-g_{\mu}\right\|_{0, \infty, R} \\
& \leqq\|g\|_{1, \infty, R} O\left(\frac{1}{\mu}\right) .
\end{aligned}
$$


It follows that

$$
\begin{aligned}
\left|\int_{\Gamma-\gamma} \frac{\partial g_{\mu}}{\partial n}\left(h_{\Gamma}^{+}-h_{\Gamma}^{-}\right) d s\right| & \leqq\|g\|_{1, \infty, R}^{2}|\Gamma-\gamma| O\left(\frac{1}{\mu}\right) \\
& \leqq\|g\|_{1, \infty, R}^{2} O\left(\frac{\log \mu}{\mu^{2}}\right) .
\end{aligned}
$$

This finishes the proof of Theorem 6.1. Note that, by a similar argument, we can also prove

$$
E\left(g_{\mu}, \varnothing\right)=E(g, \varnothing)-\frac{1}{\mu} \int_{\partial R}\left(\frac{\partial g}{\partial n}\right)^{2} d s+\|g\|_{2, \infty, R}^{2} O\left(\frac{\log \mu}{\mu^{2}}\right)
$$

We derive now the asymptotic form of the equation of first variation. We show that the first variation of $E_{\infty}(\Gamma)$ agrees with that of $E\left(f_{\Gamma}, \Gamma\right)$, at least when $\Gamma_{\text {sing }}$ is kept fixed. For simplicity, we assume that $\Gamma$ consists of $C^{2,1}$ arcs only. We shall work with an oriented piece $\gamma \subset \Gamma-\Gamma_{\text {sing }}$ and then label the left side of $\gamma$ "-", the right side " + ". This fixes the sign of the unit tangent vector $t$ to $\gamma$, the unit normal $n$ (let it point from the - side to the + side) and the curvature $\kappa$ of $\gamma$ via its definition:

$$
\frac{d t}{d s}=\kappa \cdot n
$$

THEOREM 6.2. Assume that $g$ is $C^{2,1}$. Let $\gamma$ be an oriented $C^{2,1}$-curve contained in $\Gamma$, not meeting the singularities of $\Gamma \cup \partial R$. Then for all $C^{1}$-fields $\mathbf{X}$ of normal vectors along $\Gamma$ with support contained in $\gamma$ :

(i) The first variation is given by

$$
\delta_{\mathbf{X}} E_{\infty}(\gamma)=-\int_{\gamma}\left[\mathscr{D}(g)+\frac{1}{2} \mu \nu \kappa\right](\mathbf{X} \cdot n) d s
$$

where

$$
\mathscr{D}(g)=2\left(\frac{\partial g}{\partial n} \nabla^{2} g+\frac{\partial g}{\partial s} \cdot \frac{\partial}{\partial s} \frac{\partial g}{\partial n}\right)+\kappa\left(\frac{\partial g}{\partial n}\right)^{2}
$$

(ii) There exists a constant $\rho_{\gamma}$ such that for all $\mu$ satisfying $\mu \rho_{\gamma} \geqq 6 \log (\mu|\partial R|)$ $\geqq 1$ :

(a) $e^{+}\left(f_{\Gamma}\right)-e^{-}\left(f_{\Gamma}\right)=\frac{2}{\mu} \mathscr{D}(g)+\|g\|_{3, \infty, R}^{2} O\left(1 / \mu^{2}\right)$

where $e\left(f_{\Gamma}\right)$ is the energy density corresponding to $f_{\Gamma}$.

Hence,

(b) $\delta_{\mathbf{X}} E\left(f_{\Gamma}, \Gamma\right)=(2 / \mu) \delta_{\mathbf{X}} E_{\infty}(\gamma)+\|\mathbf{X} \cdot n\|_{0, \infty, \gamma}\|g\|_{3, \infty, R}^{2} O\left(1 / \mu^{2}\right)$. 
Proof: Let $\varphi=(\mathbf{X} \cdot n)$. Let $\vec{r}(s)$ denote the parametrization of $\gamma$ by its arc length. Let $\vec{r}_{\lambda}(s)=\vec{r}(s)+\lambda \mathbf{X}(s)$. For small $|\lambda|, \vec{r}_{\lambda}$ parametrizes curves $\gamma_{\lambda}$ near $\gamma$. Let $s_{\lambda}, t_{\lambda}$ and $n_{\lambda}$ denote, respectively, the arc length, unit tangent vectors and unit normal vectors along $\gamma_{\lambda}$ :

$$
\frac{\partial \vec{r}_{\lambda}}{\partial s}=t_{0}+\lambda \frac{d \varphi}{d s} n_{0}-\lambda \varphi \kappa t_{0}
$$

Hence, up to the first-order terms in $\lambda$, we have

$$
\begin{aligned}
d s_{\lambda} & =(1-\lambda \varphi \kappa) d s \\
t_{\lambda} & =t_{0}+\lambda \frac{d \varphi}{d s} n_{0}, \\
n_{\lambda} & =n_{0}-\lambda \frac{d \varphi}{d s} t_{0} .
\end{aligned}
$$

Therefore,

$$
\begin{aligned}
\delta_{\mathbf{X}}\left[\left(\frac{\partial g}{\partial n}\right)^{2} d s-\frac{1}{2} \mu \nu d s\right]= & 2 \frac{\partial g}{\partial n}\left[\left(\delta_{\mathbf{X}} \nabla g\right) \cdot n+(\nabla g) \cdot \delta_{\mathbf{X}} n\right] d s \\
& +\left[\left(\frac{\partial g}{\partial n}\right)^{2}-\frac{1}{2} \mu \nu\right] \delta_{\mathbf{X}}(d s) \\
= & \left\{2 \frac{\partial g}{\partial n}\left[\frac{\partial^{2} g}{\partial n \partial n} \varphi-\frac{\partial g}{\partial s} \frac{d \varphi}{d s}\right]-\kappa\left[\left(\frac{\partial g}{\partial n}\right)^{2}-\frac{1}{2} \mu \nu\right] \varphi\right\} d s
\end{aligned}
$$

(where $\partial^{2} g / \partial n \partial n$ means that, at each point of $\gamma$, we take the 2-nd derivative of $g$ along the straight line normal to $\gamma$ at that point). But

$$
-\int_{\gamma} \frac{\partial g}{\partial n} \frac{\partial g}{\partial s} \frac{d \varphi}{d s} d s=\int_{\gamma}\left[\frac{\partial g}{\partial n} \frac{\partial^{2} g}{\partial s^{2}}+\frac{\partial g}{\partial s} \frac{\partial}{\partial s}\left(\frac{\partial g}{\partial n}\right)\right] \varphi d s
$$

(here $\partial^{2} g / \partial s^{2}$ means the 2-nd derivative of $g$ restricted to $\gamma$ ) and

$$
\frac{\partial^{2} g}{\partial n \partial n}+\frac{\partial^{2} g}{\partial s^{2}}=\nabla^{2} g+\kappa \frac{\partial g}{\partial n}
$$

so we get part (i) by substitution.

To prove part (ii), we now apply Part $B$ of the theorem in Appendix 3. If $\Gamma$ has arcs terminating in free ends, extend these arcs to meet $\Gamma \cup \partial R$ without creating cusps. Pick $\gamma^{*}$ such that $\gamma \subset \gamma^{*} \subset \Gamma$, $\operatorname{dist}\left(\gamma, \gamma^{*}\right)>0$ and $\gamma^{*}$ does not meet the singularities of $\Gamma \cup \partial R$. Let $\delta$ be the constant specified in the theorem in Appendix 3. (We may choose $\delta$ so that it serves all the components of $R-\bar{\Gamma}$ ). 
Let $\varepsilon=\operatorname{dist}\left(\gamma^{*}, \partial R\right)$. Let $\rho_{\gamma}=\min \{\delta, \varepsilon\}$. Let $R_{\varepsilon}=\{P \in R \mid \operatorname{dist}(P, \partial R) \geqq \varepsilon\}$. Following the method used in the proof of the lemma in Theorem 4.1, we can extend the estimates for $g_{\mu}$ as follows. For all $P \in R_{\varepsilon}$ and for all $\mu \geqq$ $3 \log (\mu|\partial R|) / \rho_{\gamma}$,

(i) for $0 \leqq k \leqq 3,\left\|g_{\mu}\right\|_{k, \infty, R_{\varepsilon}} \leqq$ (const.) $\|g\|_{k, \infty, R}$,

(ii) $\left\|g_{\mu}\right\|_{4, \infty, R_{e}} \leqq\|g\|_{3, \infty, R} O(\mu)$,

(iii) Since

$$
\begin{gathered}
g_{\mu}-g=\frac{\nabla^{2} g_{\mu}}{\mu^{2}}=\frac{\nabla^{2} g+\nabla^{2}\left(g_{\mu}-g\right)}{\mu^{2}}=\frac{\nabla^{2} g}{\mu^{2}}+\frac{\nabla^{4} g_{\mu}}{\mu^{4}} \\
g_{\mu}=g+\frac{\nabla^{2} g}{\mu^{2}}+\|g\|_{3, \infty, R} O\left(\frac{1}{\mu^{3}}\right)
\end{gathered}
$$

and

$$
\left\|g_{\mu}-g\right\|_{k, \infty, R} \leqq\|g\|_{3, \infty, R} O\left(\frac{1}{\mu^{3-k}}\right) \text { for } k=1,2
$$

Let $h_{\Gamma}=f_{\Gamma}-g_{\mu}$ as before. From Appendix 3 we get, for each component $\Omega$ of $R-\bar{\Gamma}$,

$$
\left.h_{\Gamma}\right|_{\gamma \cap \partial \Omega}=\frac{1}{\mu} \frac{\partial g_{\mu}}{\partial n}\left(1+\frac{\kappa}{2 \mu}\right)+C_{h, \gamma^{*}} O\left(\frac{1}{\mu^{3}}\right)
$$

and

$$
\left.\frac{\partial h_{\Gamma}}{\partial s}\right|_{\gamma \cap \partial \Omega}=\frac{1}{\mu} \frac{\partial}{\partial s} \frac{\partial g}{\partial n}+C_{h, \gamma^{*}} O\left(\frac{1}{\mu^{2}}\right)
$$

where

$$
C_{h, \gamma^{*}}=\left\|\frac{\partial g_{\mu}}{\partial n}\right\|_{2, \infty, \gamma^{*}}+\left\|h_{\Gamma}\right\|_{0, \infty, \partial \Omega} O\left(\frac{1}{\mu}\right)+\left\|\frac{\partial h_{\Gamma}}{\partial n}\right\|_{0,1, \partial \Omega} O\left(\frac{1}{\mu^{3}}\right)
$$

We have

$$
\begin{aligned}
& \frac{\partial g_{\mu}}{\partial n}=\frac{\partial g}{\partial n}+\|g\|_{3, \infty, R} O\left(\frac{1}{\mu^{2}}\right) \\
&\left\|\frac{\partial g_{\mu}}{\partial n}\right\|_{2, \infty, \gamma^{*}} \leqq \text { const. }\|g\|_{3, \infty, R} \\
&\left\|h_{\Gamma}\right\|_{0, \infty, \partial \Omega} \leqq 2\|g\|_{0, \infty, R} \\
&\left\|\frac{\partial h_{\Gamma}}{\partial n}\right\|_{0,1, \partial \Omega}=\|g\|_{0, \infty, R} O\left(\mu^{2}\right) .
\end{aligned}
$$


Therefore,

$$
\begin{aligned}
h^{+}-h^{-} & =\frac{2}{\mu} \frac{\partial g}{\partial n}+\|g\|_{3, \infty, R} O\left(\frac{1}{\mu^{3}}\right), \\
h^{+}+h^{-} & =\frac{\kappa}{\mu^{2}} \frac{\partial g}{\partial n}+\|g\|_{3, \infty, R} O\left(\frac{1}{\mu^{3}}\right), \\
\frac{\partial h^{ \pm}}{\partial s} & =\frac{ \pm 1}{\mu} \frac{\partial}{\partial s}\left(\frac{\partial g}{\partial n}\right)+\|g\|_{3, \infty, R} O\left(\frac{1}{\mu^{2}}\right) .
\end{aligned}
$$

Now

$$
\begin{aligned}
e^{+}\left(f_{\Gamma}\right)-e^{-}\left(f_{\Gamma}\right)= & \mu^{2}\left[\left(f_{\Gamma}^{+}-g\right)^{2}-\left(f_{\Gamma}^{-}-g\right)^{2}\right]+\left[\left(\frac{\partial f_{\Gamma}^{+}}{\partial s}\right)^{2}-\left(\frac{\partial f_{\Gamma}^{-}}{\partial s}\right)^{2}\right] \\
= & \mu^{2}\left(f_{\Gamma}^{+}-f_{\Gamma}^{-}\right)\left(f_{\Gamma}^{+}+f_{\Gamma}^{-}-2 g\right) \\
& +\left(\frac{\partial f_{\Gamma}^{+}}{\partial s}-\frac{\partial f_{\Gamma}^{-}}{\partial s}\right)\left(\frac{\partial f_{\Gamma}^{+}}{\partial s}+\frac{\partial f_{\Gamma}^{-}}{\partial s}\right) \\
= & \mu^{2}\left(h^{+}-h^{-}\right)\left(2 g_{\mu}-2 g+h^{+}+h^{-}\right) \\
& +\left(\frac{\partial h^{+}}{\partial s}-\frac{\partial h^{-}}{\partial s}\right)\left(2 \frac{\partial g_{\mu}}{\partial s}+\frac{\partial h^{+}}{\partial s}+\frac{\partial h^{-}}{\partial s}\right) .
\end{aligned}
$$

Part (ii) of the theorem follows by substitution.

\section{The Case $\mu=\infty$}

In the last section, we have argued that, in a certain sense, $E_{\infty}$ is the limiting functional of $E$ when $\mu \rightarrow \infty$ and $\frac{1}{2} \mu \nu$ has a finite limit $\nu_{\infty}$. However, the sense in question involves fixing $\Gamma$ while $\mu$ increases. To see if this is reasonable, consider the problem of minimizing the limit functional

$$
E_{\infty}(\Gamma)=\int_{\Gamma}\left(\nu_{\infty}-\left(\frac{\partial g}{\partial n}\right)^{2}\right) d x
$$

over all $\Gamma$. There are two cases:

(a) $\sup _{R}\|\nabla g\|^{2} \leqq \nu_{\infty}$.

(b) For some $P \in R,\|\nabla g(P)\|^{2}>\nu_{\infty}$.

In the first case, $E_{\infty}$ is clearly minimized by $\Gamma=\varnothing$. In the second case, we may make $\Gamma$ 's with more and more components each of which is a short arc from a 


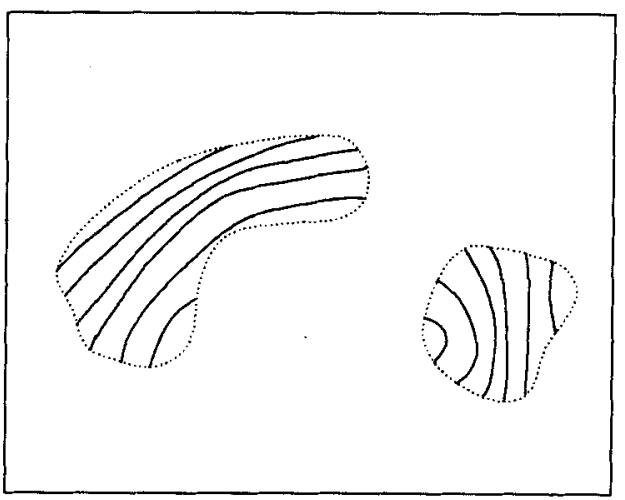

Figure 23. Conjectural form of $\Gamma, \mu$ large.

level curve of $g$ in the region $R_{0}$ where $\|\nabla g\|^{2}>\nu_{\infty}$. On such $\Gamma$ 's, inf $E_{\infty}=-\infty$, so no minimum exists!

On the other hand, we conjecture that minimizing $E$ itself is a well-posed problem. The explanation of this is that for $\mu \gg 0$ and $\mu \nu$ fixed, the minimum of $E\left(f_{\Gamma}, \Gamma\right)$ will presumably be taken on for a $\Gamma$ supported in $R_{0}$ and made up of a curve which locally has more and more components, these being smooth nearly parallel curves with a separation of about $c / \mu$ (see Figure 23). The limit of such $\Gamma$ should be taken as a current, not a curve.

Instead, the reason we are interested in the limit $E_{\infty}$ is the hope that an approximate solution to minimizing $E$ can sometimes be obtained by a very different procedure, involving two steps:

(a) smoothing $g$ by convolution with a suitable kernel of size $c / \mu$, so as to create a modified problem in which $1 / \mu$ is already small compared to fluctuations in $f$,

(b) solving for a curve $\Gamma$ which maximizes $E_{\infty}(\Gamma)$ for this smoothed $g$ and with respect to all small deformations of $\Gamma$ (as in Section 3).

Unfortunately, it is still unclear whether there is a natural class of $g$ 's for which $\Gamma$ 's satisfying (b) can be found. We hope to clarify this in a subsequent paper, and will only make a few preliminary remarks here. As we have seen in Section 4, requiring that the first variation of $E_{\infty}$ vanishes on a smooth curve $\Gamma$ is equivalent to asking that, along $\Gamma$,

$$
\frac{\partial g}{\partial n} \nabla^{2} g+\frac{\partial g}{\partial s} \frac{\partial}{\partial s}\left(\frac{\partial g}{\partial n}\right)+\frac{1}{2} \kappa\left[\left(\frac{\partial g}{\partial n}\right)^{2}+\nu_{\infty}\right]=0
$$

Now $g$ is a given function and this is not a PDE for $g$ : instead it is a 2-nd order ODE for $\Gamma$ ! To see this, let $(x(t), y(t))$ be a parametrization of $\Gamma$ and let $\theta(t)$ be 
the angle between the tangent line to $\Gamma$ and the positive $x$-axis. Then

$$
\begin{aligned}
& \frac{\partial}{\partial s}=\dot{x}(t) \frac{\partial}{\partial x}+\dot{y}(t) \frac{\partial}{\partial y}, \\
& \frac{\partial}{\partial n}=-\dot{y}(t) \frac{\partial}{\partial x}+\dot{x}(t) \frac{\partial}{\partial y} .
\end{aligned}
$$

If we let $t$ be the arc length $s$, then

$$
\begin{aligned}
(\dot{x}, \dot{y}) & =(\cos \theta, \sin \theta), \\
\dot{\theta} & =\kappa .
\end{aligned}
$$

Thus

$$
\begin{aligned}
& \frac{\partial g}{\partial n}=-\sin \theta \cdot g_{x}+\cos \theta \cdot g_{y} \\
& \frac{\partial g}{\partial s}=\cos \theta \cdot g_{x}+\sin \theta \cdot g_{y}
\end{aligned}
$$

and

$$
\begin{aligned}
\frac{\partial}{\partial s}\left(\frac{\partial g}{\partial n}\right)= & \frac{d}{d t}\left(-\sin \theta \cdot g_{x}+\cos \theta \cdot g_{y}\right) \\
= & \cos \theta \sin \theta\left(g_{y y}-g_{x x}\right)+\left(\cos ^{2} \theta-\sin ^{2} \theta\right) g_{x y} \\
& -\left(\cos \theta \cdot g_{x}+\sin \theta \cdot g_{y}\right) \kappa
\end{aligned}
$$

With these formulae we can rewrite the requirement on $\Gamma$ as the system of first-order ODE's for $(x, y, \theta)$ :

$$
\begin{aligned}
& \dot{x}=\cos \theta, \\
& \dot{y}=\sin \theta, \\
& \dot{\theta}=\frac{d_{g}^{*}(\theta) \Delta g+d_{g}(\theta) \cdot\left(\frac{1}{2} \sin 2 \theta\left(g_{y y}-g_{x x}\right)+\cos 2 \theta \cdot g_{x y}\right)}{\tau(\theta)} .
\end{aligned}
$$

where

$$
\begin{aligned}
d_{g}(\theta) & =g_{x} \cos \theta+g_{y} \sin \theta \\
d_{g}^{*}(\theta) & =-g_{x} \sin \theta+g_{y} \cos \theta \\
\tau(\theta) & =d_{g}(\theta)^{2}-\frac{1}{2} d_{g}^{*}(\theta)^{2}-\frac{1}{2} \nu_{\infty}
\end{aligned}
$$


However, we see immediately that these equations may be singular if the denominator $\tau(\theta)$ in the expression for $\dot{\theta}$ is 0 . It is better not to use arc-length parametrization, but to rewrite the equations by multiplying each equation by $\tau(\theta)$ and then choose a new "time" $t$ so that

$$
\begin{aligned}
& \dot{x}=\tau(\theta) \cos \theta, \\
& \dot{y}=\tau(\theta) \sin \theta, \\
& \dot{\theta}=(\Delta g) d_{g}^{*}(\theta)+\left(\left(g_{y y}-g_{x x}\right) \frac{1}{2} \sin 2 \theta+g_{x y} \cos 2 \theta\right) \cdot d_{g}(\theta)
\end{aligned}
$$

This form shows that the solutions are the integral curves of a smooth vector field on the unit tangent bundle $T$ to $R$. (Note that $d_{g}: T \rightarrow \mathbb{R}$ is the function on $T$ defined by the differential $d g$ and $d_{g}^{*}$ is the function on $T$ defined by the differential $(* d g)$.)

However, we may divide the unit tangent bundle $T$ into two zones:

$$
\begin{array}{cc}
\text { "space-like" zone: } & \tau(\theta)<0, \\
\text { "time-like" zone: } & \tau(\theta)>0 .
\end{array}
$$

On the boundary between these two zones, $\dot{x}=\dot{y}=0$, i.e., the vector field is "vertical". Integral curves of this field will, in general, be smooth curves on $T$, crossing from space-like to time-like and back again. But they project to curves on $R$ with smooth space-like segments that end in cusps, at which they turn into smooth time-like segments. A computer-generated example of such a curve is shown in Figure 24. It corresponds to the case $g(x, y)=e^{y}$ and $\nu_{\infty}=0$, for which all solutions are identical up to translation.

It can be shown that along its time-like segments, an integral curve locally maximizes $E_{\infty}$ whereas along its space-like segments, it locally minimizes $E_{\infty}$. For instance, if $\nu_{\infty}=0$, the gradient curves of $g$ are time-like integral curves (on gradient curves, $\partial g / \partial n \equiv 0$, hence (*) is satisfied) and these give the absolute maximum, namely 0 , of the functional

$$
E_{\infty}=-\int\left(\frac{\partial g}{\partial n}\right)^{2} d s
$$

Therefore, the existence problem for $\nu=\infty$ becomes:

Find criteria for the existence of closed everywhere space-like integral curves of $(* *)$, or singular curves, as in Section 2 , whose smooth pieces are everywhere space-like integral curves of $(* *)$.

Unfortunately, the requirement of being everywhere space-like is rather unstable. 


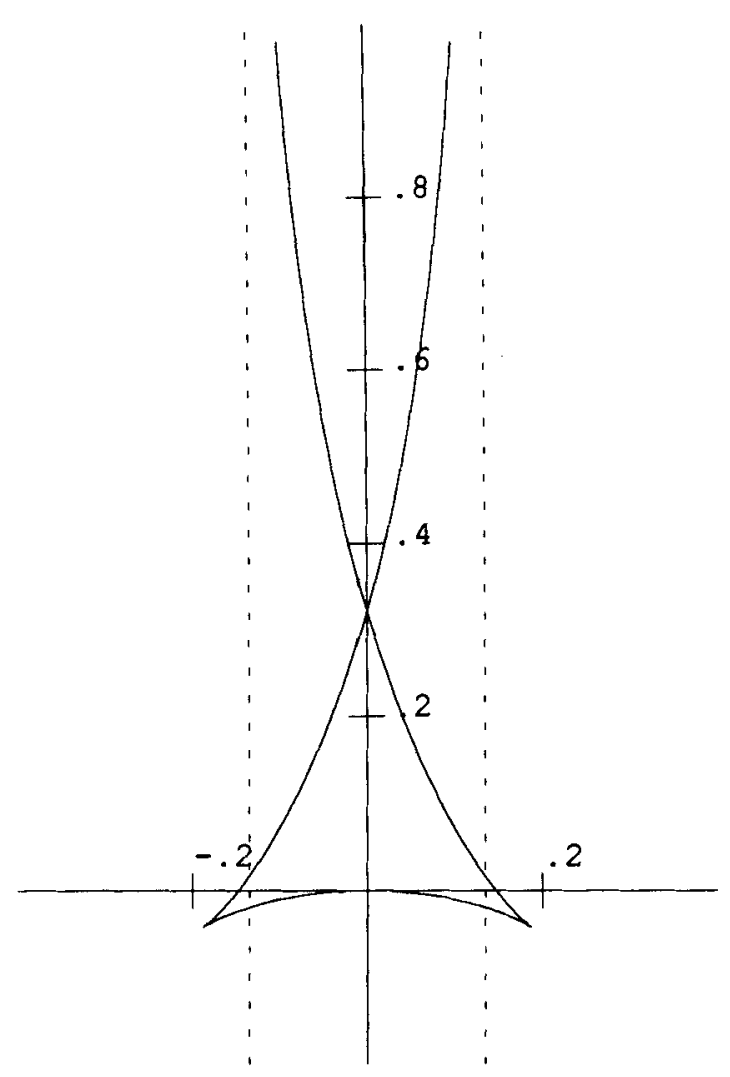

Figure 24. A solution of the ODE with "space"-and "time"-like segments.

\section{Appendix 1}

\section{Boundary Behavior of the Solutions of Neumann Problems in Non-smooth Domains}

A. We want to study the problem: given a bounded open set $\Omega$ in $\mathbb{R}^{2}$ and a function $g(x, y)$, continuous on $\bar{\Omega}$, solve for a function $f(x, y)$ such that

$$
\Delta f=\mu^{2}(f-g)
$$

$$
\left.\frac{\partial f}{\partial n}\right|_{\partial \Omega} \equiv 0
$$

If $\Omega$ has a smooth, e.g., $C^{1,1}$-boundary, it is well known that (*) has a unique solution $f$ which is $C^{1}$ and has second derivatives which are in $L_{p}$ for all $p<\infty$. If $g$ and $\partial \Omega$ are a bit smoother, then so is $f$. Unfortunately we need the 
solution $f$ for domains $\Omega$ with corners and slits and even with cusps at the end of the slits. We can find no standard reference for the existence and regularity of the solutions $f$ in this case, hence we must summarize "standard" theory here. Grisvard [7] contains all the basic ideas that we need, but they must be adapted to our particular case.

B. First of all, we use the Hilbert space $W_{2}^{1}(\Omega)$ defined in the introduction and consider the positive definite continuous quadratic function on $W_{2}^{1}(\Omega)$ :

$$
E(f)=\iint_{\Omega}\|\nabla f\|^{2} d x d y+\mu^{2} \iint_{\Omega}(f-g)^{2} d x d y
$$

By the Lax-Milgram lemma, $E$ has a unique minimum, i.e., the function $f$ such that

$$
(* *) \quad \iint_{\Omega}(\nabla \phi \cdot \nabla f) d x d y+\mu^{2} \iint \phi \cdot f d x d y=\mu^{2} \iint \phi \cdot g d x d y
$$

for all functions $\phi \in W_{2}^{1}(\Omega)$. This $f$ exists because $\phi \mapsto \mu^{2} \iint \phi \cdot g$ is a bounded linear functional on $W_{2}^{1}(\Omega)$, hence is given by dot product with some $f$.

C. Secondly, as a distribution on $\Omega, f$ satisfies $\Delta f=\mu^{2}(f-g)$. Putting $\Omega$ inside a large domain $R$ with $C^{1,1}$-boundary, let $f_{R}$ be the solution of (*) for this domain $R$. $f_{R}$ is $C^{1}$ with $L^{p}$-second derivatives for all $p$. But then, on $\Omega$, $\Delta\left(f-f_{R}\right)=\mu^{2}\left(f-f_{R}\right)$, i.e., $f-f_{R}$ is in the kernel of the elliptic operator $\Delta-\mu^{2}$. Thus $f-f_{R}$ is $C^{\infty}$. Therefore $f$ is also $C^{1}$ on $\Omega$ with $L^{p}$-second derivatives. Thus $f$ is a strong solution of $\Delta f=\mu^{2}(f-g)$ on $\Omega$, i.e., we have equality a.e. of $\Delta f$ with $\mu^{2}(f-g)$.

D. Thirdly, note that $f$ is bounded on $\Omega$ by the max and min of $g$ :

$$
\min _{\Omega} g \leqq f(x) \leqq \max _{\Omega} g \text { for all } x \in \Omega
$$

To see this, let

$$
\begin{aligned}
& f_{1}(x)=\max \left(f(x), \min _{\Omega} g\right) \\
& f_{2}(x)=\min \left(f_{1}(x), \max _{\Omega} g\right) .
\end{aligned}
$$

Then at almost all points of $\Omega$ either $f_{2}=f$ or $f_{2}$ is locally constant, hence, at 
almost all $x \in \Omega$,

$$
\left\|\nabla f_{2}\right\|^{2}(x) \leqq\|\nabla f\|^{2}(x)
$$

Moreover, at every $x \in \Omega$,

$$
\left(f_{2}(x)-g(x)\right)^{2} \leqq(f(x)-g(x))^{2}
$$

Therefore,

$$
E\left(f_{2}\right) \leqq E(f)
$$

hence $f=f_{2}$ since $f$ is the unique minimum of $E$.

E. Fourthly, look at points $P \in \partial \Omega$ such that $\partial \Omega \cap U$ is $C^{1,1}$ for some neighborhood $U$ of $P$. We claim $f$ is $C^{1}$ on $\bar{\Omega} \cap U$ and that $\partial f /\left.\partial n\right|_{\partial \Omega \cap U}=0$. To see this, introduce a subdomain $U_{P}$ of $\Omega$ which is $C^{1,1}$ and whose boundary coincides with that of $\Omega$ near $P$ (see Figure 25). Let $\eta$ be a $C^{1,1}$-function which is 1 near $P$, which is zero on $\partial U_{P}-\left(\partial \Omega \cap \partial U_{P}\right)$ and such that $\partial \eta /\left.\partial n\right|_{\partial U_{P}}=0$. Then $\tilde{f}=\eta f$ satisfies

$$
\Delta \tilde{f}-\mu^{2} \tilde{f}=\eta \mu^{2} g+2 \nabla \eta \cdot \nabla f+f \Delta \eta
$$

Since $f \in W_{2}^{1}\left(U_{P}\right), \nabla f \in L_{2}\left(U_{P}\right)$; hence

$$
\left(\Delta \tilde{f}-\mu^{2} \tilde{f}\right) \in L_{2}\left(U_{P}\right)
$$

Moreover, $\tilde{f}$ satisfies Neumann boundary conditions on $U_{P}$ weakly, in the sense

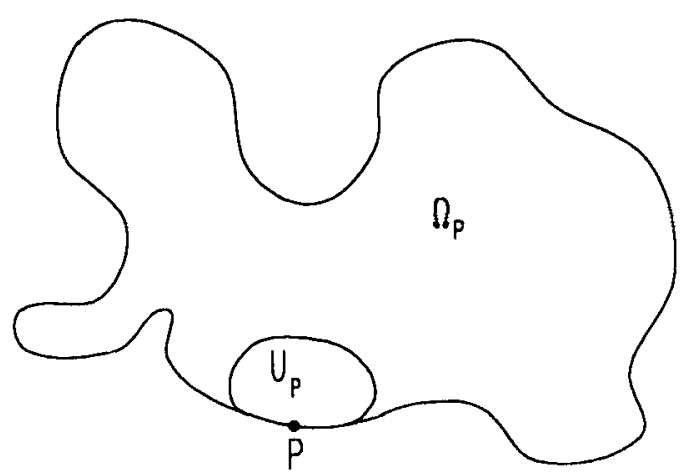

Figure 25. A smooth subdomain with same boundary near $P$. 
of Lions and Magenes, i.e.,

$$
\iint_{U_{P}} \psi \Delta \tilde{f}=\iint_{U_{p}} \tilde{f} \Delta \psi
$$

for all $\psi \in W_{2}^{2}\left(U_{P}\right)$ such that $\partial \psi /\left.\partial \nu\right|_{\partial U_{p}} \equiv 0$.

This follows from applying the basic property $(* *)$ of $f$ with $\varphi=\eta \psi$ and some juggling with Green's theorem. We apply the weak existence theorem, Proposition 2.5.2.3 in Grisvard [7], to deduce that $\tilde{f} \in W_{2}^{2}\left(U_{p}\right)$. We can soup this up a bit, because now we know that $\partial \tilde{f} / \partial x$ and $\partial \tilde{f} / \partial y$ are in the space $W_{2}^{1}\left(U_{P}\right)$, hence they are in $L_{p}\left(U_{p}\right)$ for all $p$, by the Sobolev inequalities. Now repeat the argument with $\tilde{f}=\eta^{2} f$, but starting with

$$
\Delta \tilde{f}-\mu^{2} \tilde{f}=\eta^{2} \mu^{2} g+4 \eta(\nabla \eta \cdot \nabla f)+f \Delta\left(\eta^{2}\right) \in L_{p}\left(U_{p}\right) .
$$

By the same result in Grisvard, $\tilde{f} \in W_{p}^{2}\left(U_{p}\right)$ for all $p$. This tells us that $f$ is $C^{1}$ on $\bar{\Omega}$ near $P$, by the Sobolev inequalities, and now the weak Neumann condition becomes a strong Neumann condition:

$$
\partial f /\left.\partial n\right|_{\partial \Omega}(P)=0 .
$$

F. Before studying the behavior of $u$ in corners of $\Omega$, we must make a digression on $C^{1,1}$-coordinate changes that straighten corners. We claim that for any two $C^{1,1}$-arcs meeting at $(0,0)$ with distinct tangent lines, in a neighborhood of $(0,0)$, there is a $C^{1,1}$-diffeomorphism $\Phi$ such that

(a) $\Phi$ maps the two arcs to straight rays through $(0,0)$,

(b) $d \Phi$ is the identity at $(0,0)$,

(c) $d \Phi$ carries normals to the two arcs to normals to the corresponding rays.

We prove this in two steps. In the first, we satisfy (a) and (b) but not (c). In the second, we satisfy (c) while preserving conditions (a) and (b).

Let the two arcs be

$$
y=f(x), \quad y=g(x), \quad f, g \in C^{1,1}([O, c])
$$

and

$$
\lambda=f^{\prime}(0), \quad \mu=g^{\prime}(0) \text { where } f(x)>g(x) \text { if } x \neq 0 .
$$

Define $\Phi_{1}: \mathbb{R}^{2} \rightarrow \mathbb{R}^{2}$ by

$$
\Phi_{1}(x, y)= \begin{cases}(x, y) & \text { if } x \leqq 0 \\ \left(\frac{f(x)-g(x)}{\lambda-\mu}, y+\frac{\mu f(x)-\lambda g(x)}{\lambda-\mu}\right) & \text { if } x \geqq 0 .\end{cases}
$$

$\Phi_{1}$ carries vertical lines to vertical lines. It carries $y=f(x)$ to the line $y=\lambda x$ 
and $y=g(x)$ to the line $y=\mu x$ and $d \Phi_{1}(0,0)=I_{2}$. Therefore $\Phi_{1}$ is a $C^{1,1}$-diffeomorphism in some neighborhood of $(0,0)$. Next for any $C^{0,1}$-function $h(x)$ such that $h(x)=0$ if $x \leqq 0$ and for any $\nu>0$, define $\Psi_{h, \nu}$ by

$$
\Psi_{h, \nu}(x, y)=\left(x+\int_{\nu x-2 y}^{v x-y} h(t) d t, y\right)
$$

Then

$$
\begin{aligned}
\Psi_{h, \nu}(x, 0) & =(x, 0) \\
\Psi_{h, \nu}(x, \nu x) & =(x, \nu x) \\
d \Psi_{h, \nu}(x, y) & =\left(\begin{array}{cc}
1+\nu(h(\nu x-y)-h(\nu x-2 y)) & 0 \\
2 h(\nu x-2 \eta)-h(\nu x-y) & 1
\end{array}\right)
\end{aligned}
$$

especially

$$
\begin{aligned}
d \Psi_{h, \nu}(x, \nu x) & =\left(\begin{array}{ll}
1 & 0 \\
0 & 1
\end{array}\right) \\
d \Psi_{h, \nu}(x, 0) & =\left(\begin{array}{cc}
1 & 0 \\
h(\nu x) & 1
\end{array}\right)
\end{aligned}
$$

Thus $\Psi_{h, \nu}$ is a $C^{1,1}$ diffeomorphism near $(0,0)$ which maps the sector $0<y<\nu x$ to itself, preserves perpendiculars along $y=\nu x$, but maps the perpendiculars $\partial / \partial y$ to $y=0$ to the vectors $\partial / \partial y+h(\nu x) \partial / \partial x . \Psi_{h, \nu}$ may be extended to any sector bounded by $y=0, x>0$ at one edge and including $0<y<\nu x$ by setting it equal to the identity at the new points: it is still $C^{1,1}$.

We may then use a conjugate

$$
\Phi_{2}=A^{-1} \circ \Psi_{h, \nu} \circ A
$$

where $A$ is a rotation or reflection, to modify the field of normal vectors to any sector at one edge without changing those at the other. Two such maps then will modify the two fields of normal vectors by arbitrary $C^{0,1}$-maps, so long as the normals of the apex do not move. The resulting $\Phi_{2} \circ \Phi_{1}$ can be made to have property (c).

Finally note that this argument can be extended to map the exterior sector of two $C^{1,1}$ arcs tangent at $(0,0)$ or the complement of one $C^{1,1}$-arc to the complement of a single ray by a diffeomorphism $\Phi$ with properties (a), (b) and (c) above. Let the arcs be given by

$$
y=f(x) \text { and } y=g(x)
$$


where

$$
\lambda=f^{\prime}(0)=g^{\prime}(0), \quad f(x) \geqq g(x) .
$$

The exterior sector is given by $(y>f(x)) \cup(y<g(x)) \cup(x<0)$. The complement of the $C^{1,1}$-arc is just the special case $f(x) \equiv g(x)$. To construct $\Phi$, we first use the map

$$
\Phi_{1}(x, y)= \begin{cases}(x, y) & \text { if } y \leqq 0 \\ (x, y-f(x)+\lambda x) & \text { if } y>f(x), \\ (x, y-g(x)+\lambda x) & \text { if } y<g(x)\end{cases}
$$

and then "correct" the fields of normal vectors as before.

G. We next look at singular points $P \in \partial \Omega$ which are corners with convex angles $\alpha, 0<\alpha<\pi$. At such points $P$, we claim that $f$ is still $C^{1}$ in $\bar{\Omega} \cap U$ for a suitable neighborhood $U$ of $P$, and since the two arcs of $\partial \Omega$ at $P$ have distinct tangent lines, $(\nabla f)(P)=(0,0)$. To prove this we use the techniques in Grisvard, Section 5.2, together with a $C^{1,1}$-map $\Phi$ of the type just constructed. More precisely, we assume $\Phi$ is a mapping of a neighborhood $W$ of $P$ in $\mathbb{R}^{2}$ to a neighborhood of $(0,0)$ in $\mathbb{R}^{2}$ carrying $W \cap \Omega$ to an acute triangle $\tilde{\Omega}$ in $\mathbb{R}^{2}$ and such that $d \Phi(P)=$ identity, and $\Phi$ carries normals to $W \cap \partial \Omega$ to normals to $\partial \tilde{\Omega}$ (see Figure 26). Let $\Gamma_{1}$ be the two sides of $\tilde{\Omega}$ through $(0,0)$, and $\Gamma_{2}$ the third side. Let $\eta$ be a $C^{\infty}$-function of $\|z\|$ on $\tilde{\Omega}$ which is 1 near $(0,0)$ and 0 on $\Gamma_{2}$. Consider the function

$$
\tilde{f(z)}=\eta(z) f\left(\Phi^{-1}(z)\right)
$$

on $\tilde{\Omega} ; \tilde{f}$ satisfies a variable coefficient elliptic problem on $\tilde{\Omega}$ :

$$
\begin{gathered}
A \tilde{f}=\Delta \tilde{f}+\sum b_{i j} \frac{\partial^{2} \tilde{f}}{\partial x_{i} \partial x_{j}}+\sum b_{i} \frac{\partial \tilde{f}}{\partial x_{i}}-\mu^{2} \tilde{f}=\tilde{g}, \\
\partial \tilde{f} /\left.\partial n\right|_{\Gamma_{1}} \equiv 0,\left.\quad \tilde{f}\right|_{\Gamma_{2}} \equiv 0
\end{gathered}
$$

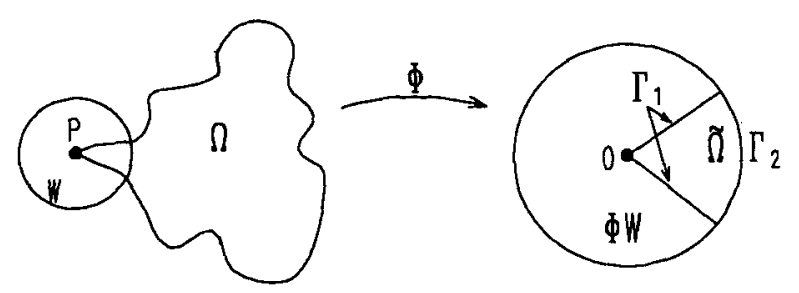

Figure 26. Straightening a corner of $\Omega$. 
for suitable bounded functions $b_{i j}, b_{i}, c$ and $\tilde{g}$ on $\tilde{\Omega}, b_{i j}$ continuous with $b_{i j}(0,0)=0, \tilde{g}$ continuous. Next choose $p$ such that

$$
2<p<\min _{\substack{\text { angles } \\ \beta \text { of } \tilde{\Omega} \\ \text { with } \beta>\pi / 2}}\left(\frac{2 \beta}{2 \beta-\pi}\right)
$$

and consider the family of operators

$$
\begin{gathered}
B_{t}: W_{p}^{2}(\tilde{\Omega}) \rightarrow L_{p}(\tilde{\Omega}) \oplus W_{p}^{1-1 / p}\left(\Gamma_{1}\right) \oplus W_{p}^{2-1 / p}\left(\Gamma_{2}\right), \\
B_{t}(\tilde{f})=\left(((1-t) A+t \Delta) \tilde{f} \partial \tilde{f} /\left.\partial n\right|_{\Gamma_{1}},\left.\tilde{f}\right|_{\Gamma_{2}}\right) .
\end{gathered}
$$

As in Grisvard, $B_{t}$ are bounded operators, $B_{1}$ is an isomorphism by Theorem 4.4.3.7. Therefore $B_{0}$ is an isomorphism. This shows $\tilde{f} \in W_{p}^{2}(\tilde{\Omega}) \subset C^{1}(\tilde{\Omega})$ using the Sobolev inequalities and the assumption $p>2$.

H. We may extend this last argument to cover corners $P$ of $\partial \Omega$ with angles $\alpha$ such that $\pi<\alpha \leqq 2 \pi$. Here $\alpha=2 \pi$ includes both the exterior sector to "cuspidal corners", pairs of $C^{1,1}$-arcs tangent at $P$ and the exterior of a single $C^{1,1}$-arc ending at $P$. However, the result is different: $f$ is not generally $C^{1}$ on $\bar{\Omega}$ near $P$. Instead $f$ has a singular leading term.

Let $z=x+i y$ be a complex coordinate on $\mathbb{R}^{2}$ and let $\theta_{1}, \theta_{2}$ be the angles with the positive $x$-axis of the tangent lines to the arcs of $\partial \Omega$ at $P\left(\theta_{1}=\theta_{2}\right.$ allowed); see Figure 27. Let

$$
f_{\text {sing }}(x, y)=\mathscr{R} e\left[e^{-i \theta_{1}}(z-z(P))\right]^{\pi / \alpha}
$$

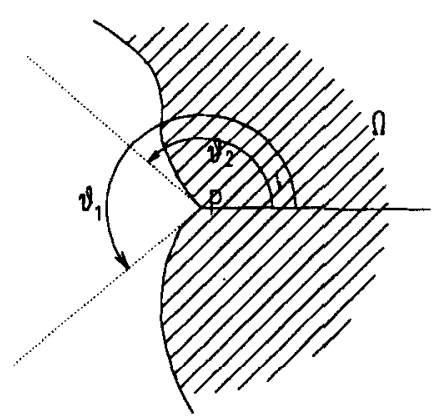

Figure 27. Orienting the sides at a corner of $\Omega$. 
Then we claim that, near $P$,

$$
f=c f_{\text {sing }}+f_{\text {reg }}, \quad \quad f_{\text {reg }} \text { a } C^{1} \text {-function. }
$$

To prove this, use a $\Phi$ carrying a neighborhood $W$ of $P$ to a polygon $\tilde{\Omega}$, an $\eta$ zero on $\partial W \cap \Omega$ and consider $\tilde{f}=\eta f \circ \Phi^{-1}$ as a function on $\tilde{\Omega}$ satisfying an elliptic boundary value problem with mixed Dirichlet-Neumann boundary conditions. Take $p$ slightly larger than 2 and use Theorem 4.4.3.7 plus the methods of Section 5.2 to deduce that, on $W_{p}^{2}$, this boundary value problem has index 1 , i.e., the map $B_{t}$ defined in subsection $\mathbf{G}$ has index 1 .

What we have to verify is that $f_{\text {sing }}$ fills in the required range, i.e.,

(i) $\left(\Delta-\mu^{2}\right) f_{\text {sing }} \in L_{p}(\Omega)$,

(ii) $\partial f_{\text {sing }} /\left.\partial n\right|_{\Gamma_{1}} \in W_{p}^{1-1 / p}\left(\Gamma_{1}\right)$.

But $f_{\text {sing }}$ is harmonic and continuous, so (i) follows.

To show (ii), let $r, \theta$ be polar coordinates at $P$ and use $r$ as a coordinate on the arcs of $\Gamma_{1}$. Take one of these and, assuming for simplicity that it is tangent to the $x$-axis, write it as

$$
y=f(r), \quad f \in C^{1,1}, f(0)=f^{\prime}(0)=0
$$

or

$$
\theta=\sin ^{-1}\left(\frac{f(r)}{r}\right)
$$

Let $g(r)=\sin ^{-1}(f(r) / r)$. Now if $f(r)$ is $C^{1,1}$ and $f(0)=0$, then $f(r) / r$ is $C^{0,1}$, hence $g(r) \in C^{0.1}$. Moreover,

$$
r g^{\prime}(r)=\frac{f^{\prime}(r)-f(r) / r}{\sqrt{1-(f(r) / r)^{2}}}
$$

which is in $C^{0,1}$ also. Now, in these coordinates,

$$
f_{\text {sing }}=r^{\pi / \alpha} \cos \left(\frac{\pi}{\alpha} \theta\right)
$$

so

$$
\begin{aligned}
\left.\frac{\partial f_{\text {sing }}}{\partial n}\right|_{\Gamma_{1}} & =\left.\left(\frac{1}{r} \frac{\partial}{\partial \theta}-r g^{\prime}(r) \frac{\partial}{\partial r}\right)\left(r^{\pi / \alpha} \cos \left(\frac{\pi}{\alpha} \theta\right)\right)\right|_{\theta=g(r)} \\
& =-\frac{\pi}{\alpha} \sin \left(\frac{\pi}{\alpha} g(r)\right) \cdot r^{\pi / \alpha-1}-\frac{\pi}{\alpha} \cos \left(\frac{\pi}{\alpha} g(r)\right)\left(r g^{\prime}(r)\right) r^{\pi / \alpha-1} \\
& =\left(C^{0,1} \text {-function) } r^{\pi / \alpha-1}\right.
\end{aligned}
$$


We use the following easy calculation:

LEMMA. If $g(x)$ is a $C^{0,1}$-function on $[0,1]$, then $g(x) x^{k} \in W_{p}^{1-1 / p}([0,1])$ if $k \geqq-\frac{1}{2}$ and $p<4$.

Differentiating $f_{\text {sing, }}$, we also note:

LEMMA. If $1 \leqq p<\frac{4}{3}$, then $f_{\text {sing }}$ and hence $f$ belongs to $W_{p}^{2}(\Omega)$.

I. Another case that we need is a point $P \in \partial \Omega$ such that $\Omega$ is the complement of an arc ending at $P$ with a cusp at $P$, i.e., an arc $\Gamma$ given by

$$
y=a x^{3 / 2}+g(x), g \in C^{1,1}, g(0)=g^{\prime}(0)=0, a \in \mathbb{R}, a \neq 0,
$$

after a rotation. This is a $C^{1,1 / 2}$-boundary point and cannot be straightened by a $C^{1,1}$-map $\Phi$. Nevertheless we claim that, near $P$, the solution $f(x, y)$ has the form

$$
f=a_{0}+a_{1} \sqrt{r} \cos \left(\frac{1}{2} \theta\right)+R(x, y),
$$

where

$$
\begin{gathered}
R \in O\left(r^{1-\varepsilon}\right), \\
\|\nabla R\| \in O\left(r^{-\varepsilon}\right) \text { for all } \varepsilon>0
\end{gathered}
$$

In particular, $f$ has a continuous extension to $\bar{\Omega}$ near $P$ (where the two sides of $\Gamma$ are considered as distinct points of $\bar{\Omega}$ ).

This can be shown by considering

$$
\tilde{f(u, v)}=f\left(u^{2}-v^{2}, 2 u v\right)
$$

defined on $\tilde{\Omega}$, one of the two components of

$$
\left\{(u, v) \mid\left(u^{2}-v^{2}, 2 u v\right) \in \Omega\right\}
$$

In complex coordinates, we are letting $x+y=(u+i v)^{2}$. This opens up the cusp into $C^{1,1}$-boundary (with a discontinuity in the curvature at $(0,0)$ ); see Figure 28 . On $\tilde{\Omega}, \tilde{f}$ satisfies

$$
\begin{gathered}
\Delta \tilde{f}=4 \mu^{2}\left(u^{2}+v^{2}\right)(\tilde{f}-\tilde{g}), \\
\partial \tilde{f} /\left.\partial n\right|_{\tilde{\Gamma}}=0 .
\end{gathered}
$$



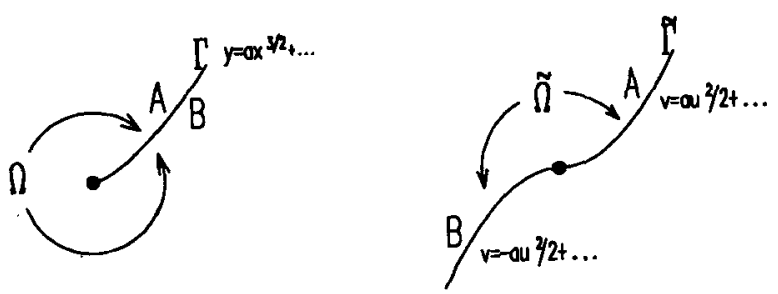

Figure 28. Smoothing a cuspidal crack by complex square root.

Therefore, as in subsection $\mathbf{G}, \tilde{f} \in W_{p}^{2}(\tilde{\Omega})$, but for all $p<\infty$. Thus $\tilde{f}$ can be written as

$$
\begin{aligned}
\tilde{f} & =a_{0}+a_{1} u+\tilde{R}, \\
\tilde{R}(0,0) & =\|\nabla \tilde{R}\|(0,0)=0 .
\end{aligned}
$$

By the Sobolev inequalities, $\tilde{R} \in C^{1, \alpha}$ and $\nabla \tilde{R} \in C^{0, \alpha}$ for all $\alpha<1$; hence

$$
\begin{aligned}
\tilde{R} & =O\left(\left(u^{2}+v^{2}\right)^{(1+\alpha) / 2}\right), \\
\|\nabla \tilde{R}\| & =O\left(\left(u^{2}+v^{2}\right)^{\alpha / 2}\right) .
\end{aligned}
$$

Going back to $f$, this gives us the expansion above.

J. One estimate is missing from our analysis: it would be nice to state it inside a cuspidal corner, i.e., in a domain

$$
g(x)<y<f(x), \quad x>0,
$$

where

$$
f, g \in C^{1,1}, \quad f(0)=g(0)=0, \quad f^{\prime}(0)=g^{\prime}(0), \quad g(x)<f(x) \quad \text { if } \quad x>0,
$$

the solution to the Neumann problem is $C^{1}$ at the corner $(0,0)$, or at least $C^{0}$. We do not know how to prove this. Fortunately we do not need it either.

\section{Appendix 2}

\section{Estimation of Neumann Problems via Brownian Motion}

Let $D$ be a domain in $\mathbb{R}^{2}$ bounded by a finite number of $C^{1,1}$-curves, meeting at corners with various angles $\alpha_{i}$ or ending at crack-tips. The case $\alpha_{i}=0$, i.e., cusps sticking out from $D$, is excluded, but $\alpha_{i}=2 \pi$, i.e., cusps sticking in, is 
allowed. Let $g \in C^{1}(\bar{D})$. We are interested in solving

$$
\begin{gathered}
\Delta f_{\mu}=\mu^{2}\left(f_{\mu}-g\right) \\
\partial f_{\mu} /\left.\partial n\right|_{\partial D} \equiv 0
\end{gathered}
$$

for various $\mu$ 's. The main result of this appendix is

THEOREM 1. Given a domain $D$ as above, there is a constant $C_{D}$ such that, for all $g$ and all $\mu$,

$$
\sup _{P \in D}\left|f_{\mu}(P)-g(P)\right| \leqq \sup _{P \in D}\|\nabla g(P)\| \cdot \frac{C_{D}}{\mu} .
$$

We know two proofs of this result. One that we found and that seems very intuitive to us which uses Brownian motion, but does require some painful estimates. The other was found by S.-T. Yau after we posed the problem to him, which is based on iterating the Sobolev inequality infinitely often to estimate $\left\|f_{\mu}-g\right\|_{p}$ for higher and higher $p$ in terms of $\left\|f_{\mu}-g\right\|_{2}$. Yau's proof demonstrates once again that things that can be proven with Brownian motion can generally be done directly. We give here our original proof because it seems so natural however.

We need Brownian motion $\vec{P}(t)$ with reflection on the boundary of $D$. The simplest way to define this process is to use the Riemann mapping theorem: Let $\Phi: D \rightarrow$ unit disk $\Delta$ be the conformal map. Then $\Phi$ extends homeomorphically from $\bar{D}$ to $\bar{\Delta}$, provided that boundary curves of $D$ which meet $D$ on both sides (e.g., edges ending in crack-tips) are counted twice as boundary curves. By a theorem of ordinary Brownian motion, $\vec{P}(t)$ on $D$ becomes Brownian motion $\vec{P}_{\Delta}(t)$ on $\Delta$ run with a new clock $\tau(t)$ :

$$
\begin{aligned}
\Phi(\vec{P}(t)) & =\vec{P}_{\Delta}(\tau(t)) \\
\tau(t) & =\int_{0}^{t}\left|\Phi^{\prime}(\vec{P}(s))\right|^{2} d s .
\end{aligned}
$$

(See e.g. [11].) On $\Delta$, define Brownian motion with reflection in the usual way and carry it back to $D$ by

$$
\begin{aligned}
\vec{P}\left(\tau^{*}(t)\right) & =\Phi^{-1}\left(\vec{P}_{\Delta}(t)\right) \\
\tau^{*}(t) & =\int_{0}^{t}\left|\left(\Phi^{-1}\right)^{\prime}\left(\vec{P}_{\Delta}(s)\right)\right|^{2} d s .
\end{aligned}
$$

Note that although $\Phi^{\prime}=0$ or $\infty$ at the corners of $D, \vec{P}_{\Delta}(t)$ hits the image of these 
corners with probability zero; hence there is no difficulty defining $\tau^{*}$ on almost all paths, and $\vec{P}(t)$ also hits a corner with probability zero.

The solution to the boundary value problem is now given by

$$
f(\vec{x})=\mathbf{E}\left[\int_{0}^{\infty} \mu^{2} g(\vec{P}(t)) \exp \left\{-\mu^{2} t\right\} d t \mid \vec{P}(0)=\vec{x}\right]
$$

For details on the construction of $\vec{P}(t)$, see [11], especially Sections $3.9,4.3$ and 4.6 , or [5], Section 1.6. For a proof of the formula, see [5], Section 2.5, Theorem 5.2 .

We need a version of Ito's lemma to calculate the expected position of $\vec{P}(t)$ :

ITo's LEMMA. Let $f$ be a $C^{1,1}$-function on a subdomain $\bar{D}_{0}$ in $\bar{D}$ such that

$$
\partial f /\left.\partial n\right|_{\bar{D}_{0} \cap \partial D} \equiv 0
$$

Then

$$
f(\vec{P}(t))=b\left(\int_{0}^{t}\|\nabla f(\vec{P}(s))\|^{2} d s\right)+\int_{0}^{t} \Delta f(\vec{P}(s)) d s,
$$

where $b$ is a one-dimensional Brownian motion.

Proof: This is Ito's lemma applied to $f(\vec{P}(t))$ : cf. [11], Section 2.9 and [5], p. 96 (note: formula $C$ there has a misprint.)

Using Ito's lemma, we now prove

THEOREM 2. Fix the domain $D$. Then there is an $r_{0}>0$ and a constant $C_{1}$ such that, for all $r \leqq r_{0}$, all $t \geqq 0$ and all $P \in D$,

$$
\mathbf{P}\left\{\begin{array}{l}
\|\vec{P}(s)-P\|>r \text { for } \\
\text { some } 0 \leqq s \leqq t, \text { given } \\
\text { that } \vec{P}(0)=P
\end{array}\right\} \leqq C_{1} \exp \left\{-r^{2} / 1600 t\right\}
$$

Proof: First of all, we need coordinates adapted to $D$. Near the $\lambda$-th edge $\Gamma_{\lambda}$ of $D$, let $d_{\lambda}(P)$ equal distance from $P$ to $\Gamma_{\lambda}$ and let $s_{\lambda}(P)$ equal arc length along $\Gamma_{\lambda}$ from the initial point of $\Gamma_{\lambda}$ to the nearest point on $\Gamma_{\lambda}$ to $P$ (cf. Figure 3 , Section 1). Note that, since $\Gamma_{\lambda}$ is $C^{1,1}, s_{\lambda}$ and $d_{\lambda}$ are $C^{1,1}$.

Secondly, near each corner $Q_{\alpha}$ of $D$, let $\Phi_{\alpha}$ be a diffeomorphism of a neighborhood of $Q_{\alpha}$ to a neighborhood of $(0,0)$ as in Appendix 1: $\Phi_{\alpha}$ carries the two edges of $D$ abutting at $Q_{\alpha}$ to two rays in $\mathbb{R}^{2}$, it carries vectors perpendicular to these edges to vectors perpendicular to the rays and its differential at $Q_{\alpha}$ is the 
identity. Choose $r_{0} \leqq r_{1}$ as follows:

(i) $\left(s_{\lambda}, d_{\lambda}\right)$ are coordinates on a neighborhood $U_{\lambda}$ of $\Gamma_{\lambda}$ defined by $d_{\lambda} \leqq r_{0}$,

(ii) the $(x, y)$-metric and the $\left(s_{\lambda}, d_{\lambda}\right)$-metric differ by at most 1.1 on this neighborhood, i.e.,

$$
d x^{2}+d y^{2} \leqq 1.1\left(d s_{\lambda}^{2}+d d_{\lambda}^{2}\right) \leqq 1.21\left(d x^{2}+d y^{2}\right),
$$

(iii) $U_{\lambda} \cap U_{\mu} \neq \varnothing$ only if $\Gamma_{\lambda}, \Gamma_{\mu}$ meet at a vertex $Q_{\alpha}$ and then $U_{\lambda} \cap U_{\mu} \subset B_{r_{1}}\left(Q_{\alpha}\right)$,

(iv) $\Phi_{\alpha}$ is defined in $B_{r_{1}}\left(Q_{\alpha}\right)$ and the $(x, y)$-metric differs from the $\left(x_{\alpha}=x \circ \Phi_{\alpha}, y_{\alpha}=y \circ \Phi_{\alpha}\right)$-metric by at most 1.1 .

Define $\gamma$ as the max of

$$
\left|\Delta s_{\lambda}\right|, \quad\left|\Delta d_{\lambda}\right|, \quad\left|\Delta d_{\lambda}^{2}\right| \text { in } U_{\lambda}
$$

and of

$$
\left|\Delta \rho_{\alpha}^{2}\right| \text { in } B_{r_{1}}\left(Q_{\alpha}\right)
$$

where $\rho_{\alpha}^{2} \equiv x_{\alpha}^{2}+y_{\alpha}^{2}$.

Now to prove the theorem, we distinguish three cases:

Case (i). $B_{2 r / 5}(P) \subset$ Int $D$.

Case (ii). The connected component of $\left(B_{2 r / 5} \cap D\right)$ containing $P$ meets exactly one edge $\Gamma_{\lambda}$ of $D$ and no corner.

Case (iii). The above component meets two edges $\Gamma_{\lambda}, \Gamma_{\mu}$ of $D$.

In case (i), we have a small square $S$ :

$$
\begin{aligned}
& x(P)-\frac{1}{5} r \leqq x \leqq x(P)+\frac{1}{5} r \\
& y(P)-\frac{1}{5} r \leqq x \leqq y(P)+\frac{1}{5} r
\end{aligned}
$$

with $S \subset B_{2 r / 5}(P) \subset B_{r}(P)$ and $S \subset \operatorname{Int}(D)$. We estimate the probability that $\vec{P}(t)$ leaves $S$ in time $t$ by adding the probabilities of it hitting the four edges of $S$ :

$$
\begin{aligned}
& \mathbf{P}\left\{\begin{array}{l}
\vec{P}(s) \notin S \text { for } \\
\text { some } 0 \leqq s \leqq t \\
\text { given } \vec{P}(0)=P
\end{array}\right\} \leqq 4 \mathbf{P}\left\{\begin{array}{l}
x(\vec{P}(s))=x(P)+\frac{1}{5} r \\
\text { for some } 0 \leqq s \leqq t \\
\text { given } \vec{P}(0)=P
\end{array}\right\} \\
&=8 \int_{r / 5}^{\infty} \frac{\exp \left\{-x^{2} / 2 t\right\}}{\sqrt{2 \pi t}} d x \\
&=8 \int_{r / 5 \sqrt{t}}^{\infty} \frac{\exp \left\{-\frac{1}{2} y^{2}\right\}}{\sqrt{2 \pi}} d y \\
& \leqq 4 \exp \left\{-r^{2} / 50 t\right\}
\end{aligned}
$$


We use here and below the simple bound (for any $a \geqq 0$ ):

$$
\int_{a}^{\infty} \frac{\exp \left\{-\frac{1}{2} y^{2}\right\}}{\sqrt{2 \pi}} d y=\exp \left\{-a^{2} h\right\} \int_{0}^{\infty} \frac{e^{-a y} \exp \left\{-\frac{1}{2} y^{2}\right\}}{\sqrt{2 \pi}} d y \leqq \frac{1}{2} \exp \left\{-\frac{1}{2} a^{2}\right\}
$$

In case (ii), we have a curvilinear rectangle $S$ around $P$ :

$$
\begin{array}{r}
s_{\lambda}(P)-\frac{1}{5} r \leqq s_{\lambda} \leqq s_{\lambda}(P)+\frac{1}{5} r \\
\max \left(0, d_{\lambda}(P)-\frac{1}{5} r\right) \leqq d_{\lambda} \leqq d_{\lambda}(P)+\frac{1}{5} r .
\end{array}
$$

Again, in this case, $S \subset B_{2 r / 5}(P) \subset B_{r}(P)$, because the furthest point of $S$ from $P$ in $d_{\lambda}, s_{\lambda}$-coordinates has distance $\sqrt{2 r} / 5$, and $\left\|\nabla d_{\lambda}\right\|,\left\|\nabla s_{\lambda}\right\| \leqq 1.1$ in $S$, hence these points have distance at most $\frac{2}{5} r$ from $P$ in $x, y$-coordinates. Now there are really two subcases:

Case (iia). $d_{\lambda}(P)>\frac{1}{5} r$.

Case (iib). $d_{\lambda}(P) \leqq \frac{1}{5} r$.

In case (iia), $S$ is in the interior of $D$ and we estimate the probability of a large excursion of $d_{\lambda}$ or $s_{\lambda}$ using Ito's lemma. Since

$$
\left|\Delta d_{\lambda}\right| \leqq \gamma \text { in } S
$$

and since $\left\|\nabla d_{\lambda}\right\| \leqq 1.1$, the lemma tells us that

$$
\mathbf{P}\left(\begin{array}{l}
d_{\lambda}(\vec{P}(s))=d_{\lambda}(P)+\frac{1}{s} r \\
\text { for some } 0 \leqq s \leqq t \\
\text { given } \vec{P}(0)=P
\end{array}\right) \leqq 2 \int_{r / s-\gamma t}^{\infty} \frac{\exp \left\{-x^{2} / 2.42 t\right\}}{\sqrt{2.42 \pi t}} d x
$$

(Note that we are adjusting for a worst case of Brownian clock speed 1.21 times normal and a maximum possible drift $\gamma t$-so that the Brownian factor must have an excursion of at least $\frac{1}{s} r-\gamma t$.) A similar estimate holds for $d_{\lambda}(\vec{P}(s))=d_{\lambda}(P)$ $-\frac{1}{5} r$ and for $s_{\lambda}(P) \pm \frac{1}{5} r$. Thus

$$
\begin{aligned}
\mathbf{P}\left(\begin{array}{l}
\vec{P}(s) \notin S \text { for } \\
\text { some } 0 \leqq s \leqq t \\
\text { given } \vec{P}(0)=P
\end{array}\right) & \leqq 8 \int_{r / 5-\gamma t}^{\infty} \frac{\exp \left\{-x^{2} / 2.42 t\right\}}{\sqrt{2.42 \pi t}} d x \\
& \leqq 8 \int_{(1 / 1.1 \sqrt{t})\left(\frac{1}{3} r-\gamma t\right)}^{\infty} \frac{\exp \left\{-\frac{1}{2} y^{2}\right\}}{\sqrt{2 \pi}} d y \\
& \leqq 4 \exp \left\{-(r-5 \gamma t)^{2} / 60.5 t\right\} .
\end{aligned}
$$


Now either $r \geqq 25 \gamma t$ or $r \leqq 25 \gamma t$. If $f \geqq 25 \gamma t$, then $r-5 \gamma t \geqq 0.8 r$; hence

$$
\mathbf{P}() \leqq 4 \exp \left\{-r^{2} / 100 t\right\} \text {. }
$$

If $r \leqq 25 \gamma t$, then $r^{2} / 100 t \leqq \frac{1}{4} \gamma r_{0}$ and hence

$$
\mathbf{P}(\quad) \leqq 1 \leqq \exp \left\{\frac{1}{4} \gamma_{0} r_{0}\right\} \exp \left\{-r^{2} / 100 t\right\} ;
$$

thus in all cases

$$
\mathbf{P}() \leqq \max \left(4, \exp \left\{\frac{1}{4} \gamma r_{0}\right\}\right) \exp \left\{-r^{2} / 100 t\right\}
$$

In case (iib), $\vec{P}(t)$ can leave $S$ in only 3 out of its 4 sides, and bounces off the 4-th. We must use $d_{\lambda}^{2}$ instead of $d_{\lambda}$ in order to satisfy $\partial /\left.\partial n\right|_{\partial D}=0$. The modified calculation shows that

$$
\begin{aligned}
& \mathbf{P}\left(\begin{array}{l}
d_{\lambda}^{2}(\vec{P}(s))=\left(d_{\lambda}(P)+\frac{1}{5} r\right)^{2} \\
\text { for some } 0 \leqq s \leqq t \\
\text { given } \vec{P}(0)=P
\end{array}\right) \\
& \quad \leqq 2 \int_{\left(d_{\lambda}(P)+\frac{1}{3} r\right)^{2}-d_{\lambda}(P)^{2}-\gamma t}^{\infty} \frac{\exp \left\{-x^{2} / 4.84\left(d_{\lambda}(P)+\frac{1}{5} r\right)^{2} 2 t\right\}}{\sqrt{2 \pi t} \cdot 2.2\left(d_{\lambda}(P)+\frac{1}{5} r\right)} d x
\end{aligned}
$$

which follows from the lemma, using $\left|\Delta\left(d_{\lambda}^{2}\right)\right| \leqq \gamma$ and

$$
\max _{S}\left\|\nabla\left(d_{\lambda}^{2}\right)\right\|^{2} \leqq 4\left(d_{\lambda}(P)+\frac{1}{5} r\right)^{2} \cdot 1.21
$$

The right side is estimated by

$$
\begin{aligned}
& 2 \exp \left\{-\left(\frac{\left(d_{\lambda}(P)+\frac{1}{5} r\right)^{2}-d_{\lambda}(P)^{2}-\gamma t}{d_{\lambda}(P)+\frac{1}{5} r}\right)^{2} / 9.68 t\right\} \\
& \quad \leqq 2 \exp \left\{-\left(\frac{1}{5} r-5 \gamma t / r\right)^{2} / 9.68 t\right\} .
\end{aligned}
$$

As in case (iia), separating the cases $r^{2} \geqq 125 \gamma t$ and $r^{2} \leqq 125 \gamma t$, we deduce that

$$
\mathbf{P}() \leqq \text { const. } \exp \left\{-r^{2} / 400 t\right\} \text {. }
$$

In case (iii), by the choice of $r_{0}$, the two edges $\Gamma_{\lambda}$ and $\Gamma_{\mu}$ meet at a corner $Q_{\alpha}$ of $D$. Again we distinguish two subcases:

Case (iiia). $Q_{\alpha} \notin B_{2 r / 5}(P)$.

Case (iiib). $Q_{\alpha} \in B_{2 r / 5}(P)$. 


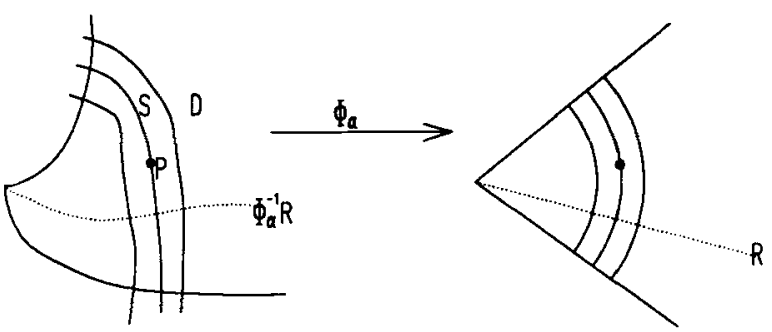

Figure 29. Neighborhoods of a point $P$ near a corner.

If the angle at the corner $Q_{\alpha}$ of $D$ is greater than $\pi$, we must be in case (iiib). We use the diffeomorphism $\Phi_{\alpha}$ defined near $Q_{\alpha}$ in both cases. Let $\rho_{\alpha}=\left\|\Phi_{\alpha}\right\|$. In case (iiia), let $S$ be the domain defined by

$$
\rho_{\alpha}(P)-\frac{1}{5} r \leqq \rho_{\alpha} \leqq \rho_{\alpha}(P)+\frac{1}{5} r
$$

see Figure 29. We claim that

$$
S \subset B_{r}(P)
$$

so that if $\vec{P}(t)$ leaves $B_{r}(P)$, it must also leave $S$ and therefore $\rho_{\alpha}(\vec{P}(t))$ must equal $\rho_{\alpha}(P) \pm \frac{1}{5} r$ at some point. To check this, use the fact that $\Phi_{\alpha}$ changes distances by at most a factor of 1.1 and some elementary geometry for the sector $\Phi_{\alpha}(S)$, depicted in Figure 30. In the figure, it is easy to check that the length of the dotted line is at most $\frac{4}{5} r$, so that

$$
\Phi_{\alpha}(S) \subset B_{4 r / 5}\left(\Phi_{\alpha}(P)\right) .
$$

Going back, $S \subset B_{r}(P)$ follows.

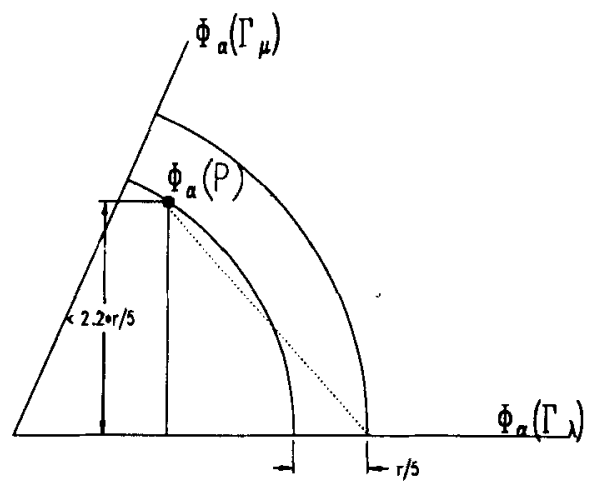

Figure 30. Estimate of certain distances. 
We now apply the lemma to the function $\rho_{\alpha}^{2}$. (Since $\Delta \rho_{\alpha} \rightarrow \infty$ at $Q_{\alpha}$, we use $\rho_{\alpha}^{2}$ instead of $\rho_{\alpha}$.) Note that

$$
\begin{aligned}
\left|\Delta\left(\rho_{\alpha}^{2}\right)\right| & \leqq \gamma \\
\left\|\nabla\left(\rho_{\alpha}^{2}\right)\right\| & =2 \rho_{\alpha}\left\|\nabla \rho_{\alpha}\right\| \leqq 2.2\left(\rho_{\alpha}(P)+\frac{1}{5} r\right) .
\end{aligned}
$$

Thus

$$
\mathbf{P}\left(\begin{array}{l}
\rho_{\alpha}(\vec{P}(s))=\rho_{\alpha}(P) \pm \frac{1}{5} r \\
\text { for some } 0 \leqq s \leqq t \\
\text { given } \vec{P}(0)=P
\end{array}\right) \leqq 2 \int_{*}^{\infty} \frac{\exp \left\{-x^{2} / 9.68\left(\rho_{\alpha}(P)+\frac{1}{5} r\right)^{2} t\right\}}{\sqrt{9.68 \pi t}\left(\rho_{\alpha}(P)+\frac{1}{5} r\right)} d x
$$

where

$$
*= \begin{cases}\left(\rho_{\alpha}(P)+\frac{1}{5} r\right)^{2}-\rho_{\alpha}(P)^{2}-\gamma t & \text { for the outer boundary, } \\ \rho_{\alpha}(P)^{2}-\left(\rho_{\alpha}(P)-\frac{1}{5} r\right)^{2}-\gamma t & \text { for the inner boundary. }\end{cases}
$$

We get as above

$$
\mathbf{P}(\quad) \leqq 2 \exp \left\{-\left[* /\left(\rho_{\alpha}(P)+\frac{1}{5} r\right)\right]^{2} / 9.68 t\right\} .
$$

But one checks that

$$
\frac{*}{\rho_{\alpha}(P)} \geqq \frac{1}{5} r-\frac{5 \gamma t}{r} \quad \text { for both boundaries, }
$$

so, as in case (iib)

$$
\mathbf{P}(\quad) \leqq \text { const. } e^{-r^{2} / 400 t} \text {. }
$$

Finally, in case (iiib), let $S$ be the domain

$$
\rho_{\alpha} \leqq \rho_{\alpha}(P)+\frac{1}{10} r .
$$

Note that $\rho_{\alpha}(Q) \leqq \rho_{\alpha}(P)+\frac{1}{10} r$ implies

$$
\begin{aligned}
\|P-Q\| & \leqq\left\|P-Q_{\alpha}\right\|+\left\|Q_{\alpha}-Q\right\| \\
& \leqq \frac{2}{5} r+1.1\left(\rho_{\alpha}(P)+\frac{1}{10} r\right) \\
& \leqq\left(\frac{2}{5}+1.1 \cdot\left(1.1 \cdot \frac{2}{5}+\frac{1}{10}\right)\right) r<r
\end{aligned}
$$

hence $S \subset B_{r}(P)$. There is only one way to leave $S$ now. Use $\rho_{\alpha}^{2}$ as in case (iiia), 
and get the estimate

$$
\mathbf{P}(\text { leaving } S) \leqq \text { const. } \exp \left\{-r^{2} / 1600 t\right\} \text { : }
$$

The main result of this appendix can now be proven using Theorem 2 and the formula for the solution $f$ of the Neumann Boundary Value Problem:

Proof: Note that

$$
g(P)=E_{\vec{P}(0)=P}\left[\int_{0}^{\infty} \mu^{2} g(\vec{P}(0)) e^{-\mu^{2} t} d t\right]
$$

hence, by the formula,

$$
\begin{aligned}
|f(P)-g(P)| & =\left|E_{\vec{P}(0)=P}\left[\int_{0}^{\infty} \mu^{2}(g(\vec{P}(t))-g(\vec{P}(0))) e^{-\mu^{2} t} d t\right]\right| \\
& \leqq \sup _{Q \in D}\|\nabla g(0)\| E_{\vec{P}(0)=P}\left[\int_{0}^{\infty} \mu^{2}\|\vec{P}(t)-\vec{P}(0)\| e^{-\mu^{2} t} d t\right]
\end{aligned}
$$

For fixed $P$ and $t$, let

$$
h(r)=\mathbf{P}[\|\vec{P}(t)-\vec{P}(0)\| \geqq r \text { if } \vec{P}(0)=P] .
$$

Then

$$
\begin{aligned}
E_{\vec{P}(0)=P}[\|\vec{P}(t)-\vec{P}(0)\|]= & -\int_{0}^{\infty} r d h(r) \\
= & \int_{0}^{\infty} h(r) d r \\
\leqq & \int_{0}^{r_{0}} C_{1} \exp \left\{-r^{2} / 1600 t\right\} d r \\
& +\int_{r_{0}}^{\operatorname{diam}(D)} C_{1} \exp \left\{-r_{0}^{2} / 1600 t\right\} d r
\end{aligned}
$$

by Theorem 2 ,

$$
\leqq 10 C_{1} \sqrt{\pi t}+C_{1} \operatorname{diam}(D) \exp \left\{-r_{0}^{2} / 1600 t\right\}
$$


Therefore,

$$
\begin{gathered}
|f(P)-g(P)| \leqq \sup \|\nabla g\| C_{1} \int_{0}^{\infty}\left(10 \sqrt{\pi t}+(\operatorname{diam} D) \exp \left\{-r_{0}^{2} / 1600 t\right\}\right) \\
\cdot \mu^{2} \exp \left\{-\mu^{2} t\right\} d t
\end{gathered}
$$

Now

$$
\int_{0}^{\infty} \sqrt{t} \mu^{2} \exp \left\{-\mu^{2} t\right\} d t=\frac{1}{\mu}\left(\int_{0}^{\infty} \sqrt{s} e^{-s} d s\right)=\frac{1}{\mu}\left(\frac{1}{2} \sqrt{\pi}\right)
$$

and

$$
\begin{aligned}
\int_{0}^{\infty} e^{-a / t}+\mu^{2} \exp \left\{-\mu^{2} . t\right\} d t & \leqq \int_{0}^{\sqrt{a} / \mu} \mu^{2} e^{-a / t} d t+\int_{\sqrt{a} / \mu}^{\infty} \mu^{2} \exp \left\{-\mu^{2} t\right\} d t \\
& =\int_{\mu / \sqrt{a}}^{\infty} a e^{-a s} d s+e^{-\sqrt{a} \mu} \\
& =2 e^{-\sqrt{a} \mu} \\
& \leqq \frac{2}{\sqrt{a} e \mu} .
\end{aligned}
$$

Thus

$$
|f(P)-g(P)| \leqq \sup \|\nabla g\| C_{1}\left(5 \pi+\frac{80 \operatorname{diam} D}{r_{0} e}\right) \frac{1}{\mu}
$$

\section{Appendix 3}

\section{Boundary Estimates with $\mu$ Large}

Let $\Omega$ be a bounded domain in $\mathbb{R}^{2}$ with Lipschitz boundary, $\partial \Omega$. In particular, $\Omega$ has no cracks. This is not a serious restriction since we can split cracked domains along the cracks and apply the results derived here to each piece separately.

For a curve $\gamma \subset \partial \Omega$, let $\partial \gamma$ be the set of end points of $\gamma$ and $|\gamma|$ be arc length of $\gamma$. For any point $P$ on $\gamma$, let

$$
\begin{aligned}
\Lambda_{\mathrm{P}}= & \text { the diameter of the largest circle through } P, \text { contained } \\
& \text { in a single component of } \mathbb{R}^{2}-\partial \Omega .
\end{aligned}
$$

Let

$$
\Lambda_{\gamma}=\min _{P \in \gamma} \Lambda_{P}
$$


If $\gamma$ is connected and contained in another connected curve $\gamma^{*}$, let

$$
d\left(\gamma, \gamma^{*}\right)=\left\{\begin{array}{l}
\infty \text { if } \gamma^{*} \text { is a closed curve, } \\
\text { arc distance between } \gamma \text { and } \partial_{\gamma}^{*} \text { otherwise. }
\end{array}\right.
$$

If $\gamma$ is $C^{1.1}$, define curvilinear coordinates in a tubular neighborhood of $\gamma$ in $\Omega$ as shown in Figure 3, Section 1: $r$ is the normal distance from $\gamma$ and $s$ is the arc length along $\gamma$. Let $n$ and $t$ denote the unit (inward) normal and tangent vectors along $\gamma$. Let $\theta$ be the angle that $t$ makes with the positive $x$-axis. If $\gamma$ is $C^{2}$, the curvature $\kappa$ along $\gamma$ is defined by the equation

$$
\frac{d n}{d s}=-\kappa t
$$

Let

$$
k_{\gamma}= \begin{cases}\frac{1}{|\partial \Omega|}+\operatorname{Lip}_{\gamma}(\theta) & \text { if } \gamma \text { is } C^{1,1}, \text { but not necessarily } C^{2,1} \\ 1 /|\partial \Omega|+\|\kappa\|_{0, \infty, \gamma}+\sqrt{\operatorname{Lip}_{\gamma}(\kappa)} & \text { if } \gamma \text { is } C^{2,1}\end{cases}
$$

where $\operatorname{Lip}_{\gamma}(\theta)$ and $\operatorname{Lip}_{\gamma}(\kappa)$ are the Lipschitz constants. For $p>1$, let $V_{\gamma, p, \mu} \subset$ $H_{p}^{2}(\Omega)$ denote the subspace of all functions $v$ such that

(a) $\nabla^{2} v-\mu^{2} v=0$ in $\Omega$ (in the weak sense),

(b) $v$ is bounded on $\partial \Omega$,

(c) $\partial v / \partial r$ is $C^{0,1}$ along $\gamma$.

Let

$$
\|v\|_{1, \gamma}=\operatorname{Lip}_{\gamma}\left(\frac{\partial v}{\partial r}\right)+k_{\gamma}\left\|\frac{\partial v}{\partial r}\right\|_{0, \infty, \gamma}+\frac{k_{\gamma}^{3}}{\mu}\|v\|_{0, \infty, \partial \Omega}+\frac{\|\partial v / \partial r\|_{0,1, \partial \Omega}}{\mu^{4}|\partial \Omega|^{6}}
$$

If $\gamma$ is $C^{2,1}$ and $\partial v / \partial r$ is $C^{1,1}$ along $\gamma$, let

$$
\begin{aligned}
\|v\|_{2, \gamma}= & \operatorname{Lip}_{\gamma}\left(\frac{\partial}{\partial s} \frac{\partial v}{\partial r}\right)+k_{\gamma}\left\|\frac{\partial}{\partial s} \frac{\partial v}{\partial r}\right\|_{0, \infty, \gamma}+k_{\gamma}^{2}\left\|\frac{\partial v}{\partial r}\right\|_{0, \infty, \gamma} \\
& +\frac{k_{\gamma}^{4}}{\mu}\|v\|_{0, \infty, \partial \Omega}+\frac{\|\partial v / \partial r\|_{0,1, \partial \Omega}}{\mu^{3}|\partial \Omega|^{6}}
\end{aligned}
$$

THEOREM. Let $\gamma \subset \gamma^{*}$ be connected $C^{1,1}$-curves $\subset \partial \Omega$ such that $\partial \gamma \cap \partial \gamma^{*}$ is empty. Then, there exists a universal constant $\beta$ such that with

$$
\delta=\min \left\{\beta / k_{\gamma^{*}}, \frac{1}{16} d\left(\gamma, \gamma^{*}\right), \frac{1}{4} \Lambda_{\gamma^{*}}\right\}
$$


for all $\mu$ satisfying $\mu \delta \geqq 6 \log (\mu|\partial \Omega|) \geqq 1$ and for all $v \in V_{\gamma^{*}, p, \mu}$, we have along $\gamma$ :

A.

$$
v(o, s)=-\frac{1}{\mu} \frac{\partial v}{\partial r}(o, s)+\frac{\varepsilon_{0}(s)}{\mu^{2}}\|v\|_{1, \gamma^{*}}
$$

where $\left|\varepsilon_{0}(s)\right|$ is bounded by a universal constant.

B. If $\gamma^{*}$ is $C^{2,1}$ and $\partial v / \partial r$ is $C^{1,1}$ along $\gamma^{*}$, then

$$
\begin{gathered}
v(o, s)=-\frac{1}{\mu}\left(1+\frac{\kappa(s)}{2 \mu}\right) \frac{\partial v}{\partial r}(o, s)+\frac{\varepsilon_{1}(s)}{\mu^{3}}\|v\|_{2, \gamma^{*}}, \\
\frac{\partial v}{\partial s}(o, s)=-\frac{1}{\mu}\left(\frac{\partial}{\partial s} \frac{\partial v}{\partial r}(o, s)\right)+\frac{\varepsilon_{2}(s)}{\mu^{2}}\|v\|_{2, \gamma^{*}},
\end{gathered}
$$

where $\left|\varepsilon_{1}(s)\right|$ and $\left|\varepsilon_{2}(s)\right|$ are bounded by universal constants.

C. Suppose that $\gamma^{*}$ is $C^{3,1}$ and $\partial v / \partial r$ is $C^{1,1}$ along $\gamma^{*}$. Then there exists a constant $A$ depending only on $\delta,|\partial \Omega|$ and $\|\kappa\|_{2, \infty, \gamma^{*}}$ such that, for all $r<\delta$,

$$
\begin{aligned}
v(r, s) & =-\frac{e^{-\mu r}}{\mu \sqrt{1-\kappa(s) r}}\left(1+\frac{\kappa(s)}{2 \mu}\right) \frac{\partial v}{\partial r}(o, s)+\frac{\varepsilon_{1}^{\prime}(r, s)}{\mu^{3}}, \\
\frac{\partial v}{\partial r}(r, s) & =\frac{e^{-\mu r}}{\mu \sqrt{1-\kappa(s) r}} \frac{\partial v}{\partial r}(o, s)+\frac{\varepsilon_{2}^{\prime}(r, s)}{\mu^{2}}, \\
\frac{\partial v}{\partial s}(r, s) & =-\frac{e^{-\mu r}}{\mu \sqrt{1-\kappa(s) r}}\left(\frac{\partial}{\partial s} \frac{\partial v}{\partial r}(o, s)\right)+\frac{\varepsilon_{3}^{\prime}(r, s)}{\mu^{2}},
\end{aligned}
$$

where, for $1 \leqq i \leqq 3$,

$$
\left|\varepsilon_{i}^{\prime}(r, s)\right| \leqq A(1+\mu r) e^{-\mu r}\left(\left\|\frac{\partial v}{\partial r}\right\|_{2, \infty, r^{*}}+\frac{\|v\|_{0, \infty, \partial \Omega}+\|\partial v / \partial r\|_{0,1, \partial \Omega}}{\mu}\right) .
$$

In order to make the proof more readable we give a proof only for Part B of the theorem. The proof of Part A is essentially contained in the proof of Part B except that we have to replace all the $C^{2,1}$-estimates of $\gamma^{*}$ by $C^{1,1}$-estimates and develop the error terms up to one order of $1 / \mu$ less. Part $C$ is not needed in our 
application of the theorem in this paper. Its proof is analogous to the proof of Part B involving a few more calculations of the same type.

The theorem is proved by estimating the integrals in Green's integral representation formula which we now recall. By Sobolev imbedding theorem,

$$
v \in H_{q}^{1}(\Omega) \text { where } q>2
$$

and by the trace theorems (see [7]),

$$
\frac{\partial v}{\partial n} \in L_{q}(\partial \Omega)
$$

The assumption that $\left(-\nabla^{2}+\mu^{2}\right) v=0$ in the weak sense means that

$$
\begin{gathered}
\int_{\Omega}\left(\nabla \phi \cdot \nabla v+\mu^{2} \phi v\right)+\int_{\partial \Omega} \phi \frac{\partial v}{\partial n}=0 \text { for all } \phi \in H_{q^{\prime}}^{1}(\Omega), \\
\text { where } \frac{1}{q}+\frac{1}{q^{\prime}}=1 .
\end{gathered}
$$

Fix a point $P \in \mathbb{R}^{2}$ and let $\phi=K_{0}(\mu \mid P Q)$, where $K_{0}$ is the zeroth-order modified Bessel function of the second kind and $Q \in \mathbb{R}^{2}$. We get

$$
\int_{\Omega_{Q}}\left(\nabla_{Q} v \cdot \nabla_{Q} K_{0}+\mu^{2} v_{Q} K_{0}\right)+\int_{\partial \Omega_{Q}} K_{0} \frac{\partial v}{\partial n_{Q}}=0
$$

$K_{0}$ satisfies the equation

$$
\left(-\nabla_{Q}^{2}+\mu^{2}\right) K_{0}(\mu|P Q|)=2 \pi \omega_{P}
$$

in the weak sense, where $\omega_{P}$ is the delta function concentrated at $P$. Therefore, for all $v \in V_{\gamma, p, \mu}$,

$$
\int_{\Omega_{Q}}\left(-\nabla_{Q}^{2}+\mu^{2}\right) K_{0}(\mu|P Q|) v(Q)=2 \pi \int_{\Omega_{Q}} \omega_{P} v(Q) .
$$

If $P \notin \partial \Omega$ or if $P \in \partial \Omega$ where it is at least $C^{1,1}$, then $\partial K_{0} / \partial n_{Q}$ is bounded in a neighborhood of $P$ and hence, by applying the Green's formula to $\Omega-$ ( $\varepsilon$-neighborhood of $P$ ) and then taking the limit as $\varepsilon \rightarrow 0$, we get

$$
\int_{\Omega_{Q}}\left(\nabla_{Q^{v}} \cdot \nabla_{Q} K_{0}+\mu^{2} v_{Q} K_{0}\right)+\int_{\partial \Omega_{Q}} \frac{\partial K_{0}}{\partial n_{Q}} v_{Q}=2 \pi \int_{\Omega_{Q}} \omega_{P} v_{Q}
$$

for all $v \in V_{\varnothing, p, \mu}$ and all $P$ in whose neighborhood $\partial \Omega$ is $C^{1,1}$. Subtracting (1) 
from (2), we get

$$
\begin{aligned}
& \int_{\Omega_{Q}} \omega_{P} v_{Q}=\frac{1}{2 \pi} \int_{\partial \Omega_{Q}}\left(-K_{0} \frac{\partial v}{\partial n_{Q}}+\frac{\partial K_{0}}{\partial n_{Q}} v_{Q}\right), \\
& \int_{\Omega_{Q}} v_{Q} \omega_{P}=m_{P} v(P),
\end{aligned}
$$

where

$$
m_{P}= \begin{cases}1 & \text { if } P \in \bar{\Omega}-\partial \Omega \\ \frac{1}{2} & \text { if } P \in \partial \Omega \text { and } \partial \Omega \text { is } C^{1,1} \text { at } P,\end{cases}
$$

since $v$ is continuous at $P$.

We have

(3) $m_{P} v(P)=\frac{1}{2 \pi} \int_{\partial \Omega_{Q}}\left(-K_{0} \frac{\partial v}{\partial n_{Q}}+\frac{\partial K_{0}}{\partial n_{Q}} v(Q)\right)$ where $m_{P}=\int_{\Omega_{Q}} \omega_{P}$.

Our task is to estimate the integrals in the integral equation above and solve explicitly for $v$ in terms of $\partial v / \partial n$. The key point is that $K_{0}$ and its derivatives decrease exponentially with increasing $\mu|P Q|$ and hence the values of the integrals when $\mu$ is large depend essentially on a small segment of $\partial \Omega$ near $P$ which may be approximated by a circle.

We prove a series of lemmas first. In Lemma 1, we derive geometric estimates which depend only on $\Omega$. We use these estimates in Lemma 2 to specify $\delta$. In Lemma 3 , we derive some basic formulae for $K_{0}$, Lemma 4 lists estimates for the basic integrals that arise when we try to estimate the integrals in the Green's formula and their derivatives.

Define $R: \mathbb{R}^{2} \times \partial \Omega \rightarrow \mathbb{R}_{\geq 0}$ by setting $R(P, Q)=$ distance between $P \in \Omega$ and $Q \in \partial \Omega$. If $P$ and $Q$ lie in a tubular neighborhood of a connected curve $\gamma \subset \partial \Omega$ so that $P$ and $Q$ have coordinates $\left(r, s_{P}\right)$ and $\left(0, s_{Q}\right)$, respectively, then $R$ becomes a function of $r, s_{P}$ and $s_{Q}$. Define $\sigma: \gamma \times \gamma \rightarrow \mathbb{R}_{\geqq 0}$ by setting $\sigma(P, Q)=\left|s_{Q}-s_{P}\right|$. Let $\partial_{\sigma}$ denote the symmetric operator $\partial / \partial s_{P}+\partial / \partial s_{Q}$. Let $n_{P}, t_{P}$ and $\kappa_{P}$ denote the normal vector, tangent vector and the curvature at the point $\left(0, s_{P}\right)$. Let $n_{Q}, t_{Q}$ and $\kappa_{Q}$ be the corresponding quantities at $Q$. In what follows, if an estimate contains an order term $O\left(\varepsilon^{m}\right)$ in variable $\varepsilon$, then $\left|O\left(\varepsilon^{m}\right) / \varepsilon^{m}\right|$ is bounded by a universal constant.

Lemma 1. I. Let $x_{1}, x_{2}$ be the coordinates in $\mathbb{R}^{2}$ and let $D_{i}$ denote the operator $\partial / \partial x_{i}$. Let $q \in \partial \Omega$ be such that $n_{Q}$ is defined. Then for all $P \in \mathbb{R}^{2}-\{Q\}$, 
$i=1,2$ and $j=1,2$,

$$
\begin{array}{r}
\left|D_{i_{P}} R\right| \leqq 1, \\
\left|\frac{\partial R}{\partial n_{Q}}\right| \leqq 1, \\
\left|D_{i_{P}} \partial R / \partial n_{Q}\right| \leqq \frac{1}{R} .
\end{array}
$$

II. Suppose that $P$ belongs to a tubular neighborhood of a connected $C^{2,1}$-curve $\gamma \subset \partial \Omega$ and $Q \in \gamma$. Then, for all $r, \sigma \leqq 1 / k_{\gamma}$, we have the following estimates:

$$
\begin{aligned}
R^{2} & =r^{2}+\sigma^{2}\left(1-\kappa_{P} r\right)+k_{\gamma}^{2}\left[r O\left(\sigma^{3}\right)+O\left(\sigma^{4}\right)\right] \\
R \frac{\partial R}{\partial r} & =r-\frac{1}{2} \kappa_{P} \sigma^{2}+k_{\gamma}^{2} O\left(\sigma^{3}\right) \\
R \frac{\partial R}{\partial s_{P}} & =-\left(1-\kappa_{P} r\right)\left(s_{Q}-s_{P}\right)+k_{\gamma}^{2} O\left(\sigma^{3}\right) \\
R \frac{\partial R}{\partial s_{Q}} & =\left(1-\kappa_{P} r\right)\left(s_{Q}-s_{P}\right)+k_{\gamma}^{2}\left[r O\left(\sigma^{2}\right)+O\left(\sigma^{3}\right)\right] \\
\partial_{\sigma} R^{2} & =k_{\gamma}^{2}\left[r O\left(\sigma^{2}\right)+O\left(\sigma^{3}\right)\right]
\end{aligned}
$$

(vii) $\frac{\partial}{\partial r}\left(R \frac{\partial R}{\partial n_{Q}}\right)=-1+\frac{1}{2} \kappa_{P}^{2} \sigma^{2}+k_{\gamma}^{2} O\left(\sigma^{3}\right)$,

(viii) $\frac{\partial}{\partial s_{P}}\left(R \frac{\partial R}{\partial n_{Q}}\right)=\kappa_{P}\left(1-\kappa_{P} r\right)\left(s_{Q}-s_{P}\right)+k_{\gamma}^{2} O\left(\sigma^{2}\right)$,

(ix) $\partial_{\sigma}\left(R \frac{\partial R}{\partial n_{Q}}\right)=k_{\gamma}^{2} O\left(\sigma^{2}\right)$.

(If $\gamma$ is only $C^{1,1}$, the corresponding estimates may be obtained by dropping the terms containing $\kappa_{P}$ and replacing $k_{\gamma}^{2} O\left(\sigma^{m}\right)$ by $k_{\gamma} O\left(\sigma^{m-1}\right)$. We leave it to the reader to verify this.)

Proof: Part I may be proved directly by differentiating $R$. (It is convenient to let the $x_{1}$-axis coincide with $n_{Q}$ ) 
Consider now Part II. Let $P_{0}$ be the point with coordinates $\left(0, s_{P}\right)$ and let $m$ be the point on $\gamma$ with coordinates $\left(0, \frac{1}{2}\left(s_{P}+s_{Q}\right)\right)$. From the error formulae for divided differences, we obtain the following estimates in which $k_{\gamma}^{2} O\left(\sigma^{m}\right)$ is abbreviated as $O_{1}\left(\sigma^{m}\right)$ :

$$
\overrightarrow{P_{0} Q}=\left(s_{Q}-s_{P}\right)\left[t_{m}+\overrightarrow{O_{1}\left(\sigma^{2}\right)}\right]
$$

where $t_{m}$ is the tangent vector at $m$ and $\overrightarrow{O_{1}\left(\sigma^{2}\right)}$ is a vector of magnitude $O_{1}\left(\sigma^{2}\right)$,

$$
\begin{aligned}
n_{Q}-n_{P} & =\left(s_{Q}-s_{P}\right)\left[-\kappa_{m} t_{m}+\overrightarrow{O_{1}(\sigma)}\right] \\
& =\left(s_{Q}-s_{P}\right)\left[-\kappa_{P} t_{P}+\overrightarrow{O_{1}(\sigma)}\right]=\left(s_{Q}-s_{P}\right)\left[-\kappa_{Q} t_{Q}+\overrightarrow{O_{1}(\sigma)}\right] \\
t_{Q}-t_{P} & =\left(s_{Q}-s_{P}\right)\left[\kappa_{m} n_{m}+\overrightarrow{O_{1}(\sigma)}\right] \\
& =\left(s_{Q}-s_{P}\right)\left[\kappa_{P} n_{P}+\overrightarrow{O_{1}(\sigma)}\right]=\left(s_{Q}-s_{P}\right)\left[\kappa_{Q} n_{Q}+\overrightarrow{O_{1}(\sigma)}\right] \\
n_{Q}+n_{P} & =2 n_{m}+\overrightarrow{O_{1}\left(\sigma^{2}\right)} \\
t_{Q}+t_{P} & =2 t_{m}+\overrightarrow{O_{1}\left(\sigma^{2}\right)}
\end{aligned}
$$

From these estimates, we obtain, in turn, the following:

$$
\begin{aligned}
\overrightarrow{P_{0} Q} \cdot \overrightarrow{P_{0} Q} & =\sigma^{2}+O_{1}\left(\sigma^{4}\right), \\
\overrightarrow{P_{0} Q} \cdot n_{P} & =\frac{1}{2} \kappa_{m} \sigma^{2}+O_{1}\left(\sigma^{3}\right), \\
\overrightarrow{P_{0} Q} \cdot n_{Q} & =-\frac{1}{2} \kappa_{m} \sigma^{2}+O_{1}\left(\sigma^{3}\right), \\
\overrightarrow{P_{0} Q} \cdot t_{P} & =\left(s_{Q}-s_{P}\right)+O_{1}\left(\sigma^{3}\right), \\
\overrightarrow{P_{0} Q} \cdot t_{Q} & =\left(s_{Q}-s_{P}\right)+O_{1}\left(\sigma^{3}\right), \\
\overrightarrow{P_{0} Q} \cdot\left(t_{Q}-t_{P}\right) & =O_{1}\left(\sigma^{3}\right), \\
n_{P} \cdot t_{Q} & =-n_{Q} \cdot t_{P}=\kappa_{P}\left(s_{Q}-s_{P}\right)+O_{1}\left(\sigma^{2}\right), \\
1-n_{P} \cdot n_{Q} & =\frac{1}{2} \kappa_{P}^{2} \sigma^{2}+k_{\gamma} O_{1}\left(\sigma^{3}\right) .
\end{aligned}
$$


The estimates in Part II now follow by substituting the above estimates into the following formulae for $R^{2}$ and its derivatives:

$$
\begin{aligned}
R^{2} & =\left(-r n_{P}+\overrightarrow{P_{0} Q}\right) \cdot\left(-r n_{P}+\overrightarrow{P_{0} Q}\right) \\
R \frac{\partial R}{\partial r} & =r-\overrightarrow{P_{0} Q} \cdot n_{P} \\
R \frac{\partial R}{\partial s_{P}} & =-\left(1-\kappa_{P} r\right) \overrightarrow{P_{0} Q} \cdot t_{P} \\
R \frac{\partial R}{\partial s_{Q}} & =-r n_{P} \cdot t_{Q}+\overrightarrow{P_{0} Q} \cdot t_{Q} \\
R \frac{\partial R}{\partial n_{Q}} & =-r n_{P} \cdot n_{Q}+\overrightarrow{P_{0} Q} \cdot n_{Q} \\
\frac{\partial}{\partial r}\left(R \frac{\partial R}{\partial n_{Q}}\right) & =-n_{P} \cdot n_{Q} \\
\frac{\partial}{\partial s_{P}}\left(R \frac{\partial R}{\partial n_{Q}}\right) & =-\left(1-\kappa_{P} r\right) t_{P} \cdot n_{Q} \\
\frac{\partial}{\partial s_{Q}}\left(R \frac{\partial R}{\partial n_{Q}}\right) & =-\kappa_{Q}\left(-r n_{P}+\overrightarrow{P_{0} Q}\right) \cdot t_{Q}
\end{aligned}
$$

LEMma 2. There exists a universal constant $\beta \leqq \frac{1}{2}$ such that if $\gamma$ is a connected $C^{1,1}$-curve $\subset \partial \Omega$ and if we choose $\delta \leqq \beta / k_{\gamma}$, then

(a) for all points $P$ in a tubular neighborhood of $\gamma$ for which $r \leqq \delta$ and $\{Q \in \partial \Omega \mid \sigma \leqq 4 \delta\} \subset \gamma$, we have

$$
\begin{array}{r}
r^{2}+4 \sigma^{2} \geqq R^{2} \geqq r^{2}+\frac{1}{4} \sigma^{2}, \\
2 \sigma \geqq\left|R \frac{\partial R}{\partial s_{Q}}\right| \geqq \frac{1}{2} \sigma .
\end{array}
$$

(b) If, furthermore, $\delta \leqq \frac{1}{4} \Lambda_{\gamma}$, then $R \geqq 2 \delta$ whenever $\sigma \geqq 4 \delta$ or $Q \notin \gamma$.

Proof: The estimates for $R^{2}$ and $R \partial R / \partial s_{Q}$ in Lemma 1 imply the existence of $\beta$ such that the inequalities in part (a) are satisfied. Suppose now that $\delta \leqq \frac{1}{4} \Lambda_{\gamma}$. Let $S$ be the circle with radius $2 \delta$ and center at $P$. Let $Q_{1}, Q_{2}, q_{1}, q_{2}$ be the points as shown in Figure 31 . Since $R \geqq 2 \delta$ when $\sigma=4 \delta,\left|s_{Q_{i}}-s_{P}\right| \leqq 4 \delta$ for $i=1,2$. Therefore, $Q_{1}$ and $Q_{2}$ lie on $\gamma$. For $i=1,2$, the arc $Q_{i} q_{i}$ must lie inside the circle of diameter $\Lambda_{\gamma}$, tangent to $\gamma$ at $Q_{i}$, since $4 \delta<\Lambda_{\gamma}$. The rest of 


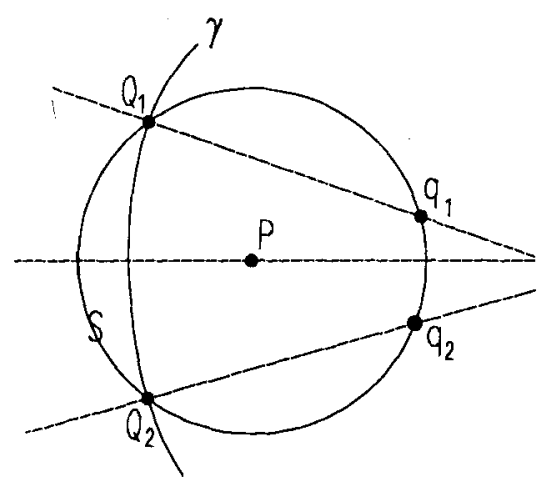

Figure 31. Definition of $Q_{i}$ and $q_{i}, i=1$ and 2 .

the circle $S$ must cut any normal to a point on $\gamma$ between $Q_{1}$ and $Q_{2}$ at a distance at most $4 \delta<\Lambda_{\gamma}$. It follows that $\partial \Omega \cap S=\left\{Q_{1}, Q_{2}\right\}$. Any ray from $P$ to a point on $\partial \Omega$ with $\sigma \geqq 4 \delta$ must intersect $S$ first and hence, has length at least $2 \boldsymbol{\delta}$.

LEMMA 3. Let $K_{0}(z)$ be the zeroth-order modified Bessel function of the second $k i n d$. Let $L$ denote the operator $-(1 / z) d / d z$. Then:

(i) $L z^{2} L K_{0}(z)=K_{0}(z)$.

(ii) There exists a constant $c$ such that, for $0 \leqq z<\infty$,

$$
\begin{aligned}
& 0 \leqq z^{1 / 2} K_{0}(z) \leqq c e^{-z} \\
& 0 \leqq z^{2} L K_{0}(z) \leqq c \max (1, \sqrt{z}) e^{-z}
\end{aligned}
$$

(iii) For any integers $m, n \geqq 0$, there exist constants $c_{n}$ and $d_{m, n}$ such that, for all $z \geqq 1$,

$$
\begin{aligned}
& (-1)^{n} \frac{d^{n}}{d z^{n}} K_{0}(z) \leqq c_{n} z^{-1 / 2} e^{-z}, \quad L^{n} K_{0}(z) \leqq c_{n} z^{-n-1 / 2} e^{-z}, \\
& \int_{z}^{\infty} z^{m} L^{n} K_{0}(z) d z \leqq d_{m, n} z^{m-n-1 / 2} e^{-z}
\end{aligned}
$$

Proof: Part (i) follows from the identities

$$
\begin{gathered}
\frac{d}{d z} K_{0}(z)=-K_{1}(z), \\
\frac{d}{d z}\left(z K_{1}(z)\right)=-z K_{0}(z),
\end{gathered}
$$


where $K_{1}(z)$ is the first-order modified Bessel function of the second kind. (For a collection of formulae involving Bessel functions, see the Handbook of Mathematical Functions, [2], vol. 55.) For proving part (ii), use the polynomial approximations for $z^{1 / 2} K_{0}(z)$ and $z K_{1}(z)$ in the range $0 \leqq z \leqq 2$ and $2 \leqq z<\infty$. The last part follows from (i) and (ii) by differentiation and integration by parts.

LEMMA 4. Let $\gamma$ be a connected $C^{2,1}$-curve $\subset \partial \Omega$. Let $j$ be an integer, $0 \leqq j \leqq 2$. Choose $\delta \leqq \beta / k_{\gamma}$ where $\beta$ is the constant as in Lemma 2. Let $P$ with coordinates $\left(r, s_{P}\right)$ be a point in a tubular neighborhood of $\gamma$ with $r<\delta$. Let $N_{P}=\{Q \in \partial \Omega \mid \sigma<4 \delta\}$ and assume that $N_{P} \subset \gamma$. Let $\Phi \in L_{1}(\partial \Omega)$ be a function on $\partial \Omega$ which is $C^{j-1,1}$ in $\gamma$. Let

$$
\begin{aligned}
\Phi_{P} & =\Phi\left(s_{P}\right), \\
C_{0, \gamma}^{\Phi} & =\|\Phi\|_{0, \infty, \gamma}, \\
C_{1, \gamma}^{\Phi} & =k_{\gamma}\|\Phi\|_{0, \infty, \gamma}+\operatorname{Lip}_{\gamma}(\Phi),
\end{aligned}
$$

and

$$
C_{2, \gamma}^{\Phi}=k_{\gamma}^{2}\|\Phi\|_{0, \infty, \gamma}+k_{\gamma}\left\|\frac{d \Phi}{d s}\right\|_{0, \infty, \gamma}+\operatorname{Lip}_{\gamma}\left(\frac{d \Phi}{d s}\right)
$$

Let $\mathscr{L}$ denote the operator $-(1 / R) d / d R$. Then, there exist universal constants $C_{1}, C_{2}, \cdots, C_{1}^{\prime}, C_{2}^{\prime}, \cdots, C^{\prime \prime}, C_{1}^{\prime \prime}, C_{2}^{\prime \prime}, \cdots, \alpha_{0}$, and polynomials $\alpha_{1}(\mu r)$ and $\alpha_{3}(\mu r)$ of degrees 1 and 3 in variable $\sqrt{\mu r}$, respectively, with universal coefficients such that the following estimates hold for all $\mu$ satisfying $\mu \delta \geqq 6 \log (\mu|\partial \Omega|) \geqq 1$ :

$$
\frac{1}{2 \pi} \int_{N_{P}} K_{0}(\mu R) \Phi d s_{Q} \approx \frac{\Phi_{p} e^{-\mu r}}{2 \mu \sqrt{1-\kappa_{P} r}}
$$

$$
\text { with absolute error } \leqq \begin{cases}\frac{C_{1, \gamma}^{\Phi}}{\mu^{2}} \alpha_{1}(\mu r) e^{-\mu r} & \text { if } j=1 \\ \frac{C_{2, \gamma}^{\Phi}}{\mu^{3}} \alpha_{3}(\mu r) e^{-\mu r} & \text { if } j=2\end{cases}
$$


672

D. MUMFORD AND J. SHAH

$$
\frac{1}{2 \pi} \int_{N_{p}} \mathscr{L} K_{0}(\mu R) \Phi d s_{Q} \approx \frac{\Phi_{P} e^{-\mu r}}{2 r \sqrt{1-\kappa_{P} r}}
$$

$$
\text { with absolute error } \leqq \begin{cases}\frac{C_{1, \gamma}^{\Phi}}{\sqrt{\mu r}} \alpha_{1}(\mu r) e^{-\mu r} & \text { if } j=1, \\ \frac{C_{2, \gamma}^{\Phi}}{\mu} \alpha_{3}(\mu r) e^{-\mu r} & \text { if } j=2,\end{cases}
$$

$$
\begin{aligned}
\frac{1}{2 \pi} \int_{N_{P}} \mathscr{L} K_{0}(\mu R) \sigma^{2} \Phi d s_{Q} & \approx \frac{\Phi_{P} e^{-\mu r}}{2 \mu\left(1-\kappa_{P} r\right)^{3 / 2}} \\
\text { with absolute error } \leqq & \left\{\begin{array}{l}
\frac{C_{1, \gamma}^{\Phi}}{\mu^{2}} \alpha_{1}(\mu r) e^{-\mu r} \quad \text { if } j=1, \\
\frac{C_{2, \gamma}^{\Phi}}{\mu^{3}} \alpha_{3}(\mu r) e^{-\mu r} \quad \text { if } j=2,
\end{array}\right. \\
\frac{1}{2 \pi} \int_{N_{P}} \mathscr{L}^{2} K_{0}(\mu R) \Phi d s_{Q} & \approx \frac{\Phi_{P}(1+\mu r) e^{-\mu r}}{2 r^{3} \sqrt{1-\kappa_{P} r}}
\end{aligned}
$$

with absolute error $\leqq \begin{cases}\frac{C_{1, \gamma}^{\Phi}}{r^{2}} \alpha_{1}(\mu r) e^{-\mu r} & \text { if } j=1, \\ \frac{C_{2, \gamma}^{\Phi}}{r} \alpha_{3}(\mu r) e^{-\mu r} & \text { if } j=2\end{cases}$

$$
\frac{1}{2 \pi} \int_{N_{P}} \mathscr{L}^{2} K_{0}(\mu R) \sigma^{2} \Phi d s_{Q} \approx \frac{\Phi_{P}}{2 r} e^{-\mu r}
$$

$$
\text { with absolute error } \leqq \frac{C_{1, \gamma}^{\Phi}}{\sqrt{\mu r}} \alpha_{1}(\mu r) e^{-\mu r} \quad \text { if } j=1 \text {, }
$$

$$
\begin{array}{rlrl}
\left|\int_{N_{p}} K_{0}(\mu R) \sigma^{2} \Phi d s_{Q}\right| & \leqq \frac{C_{0, \gamma}^{\Phi}}{\mu^{3}}(1+\mu r) \alpha_{0} e^{-\mu r}, & j=0, \\
\left|\int_{N_{P}} \mathscr{L} K_{0}(\mu R) \sigma^{4} \Phi d s_{Q}\right| \leqq \frac{C_{0, \gamma}^{\Phi}}{\mu^{3}}(1+\mu r) \alpha_{0} e^{-\mu r}, & j=0, \\
\left|\int_{N_{p}} \mathscr{L}^{2} K_{0}(\mu R) \sigma^{4} \Phi d s_{Q}\right| \leqq \frac{C_{0, \gamma}^{\Phi}}{\mu} \alpha_{0} e^{-\mu r}, & j=0 .
\end{array}
$$

If $r=0$, then for $m, n \geqq 0, j=0$,

$$
\left|\int_{N_{p}} \mathscr{L}^{n} K_{0}(\mu R) \sigma^{2 n+m} \Phi d s_{Q}\right| \leqq \frac{C_{n}}{\mu^{m+1}} C_{0, \gamma}^{\Phi} .
$$


For $m, n \geqq 0$ and $j=1$,

$$
\lim _{r \rightarrow 0}\left|\int_{N_{p}} \mathscr{L}^{n} K_{0}(\mu R) \sigma^{2 n+m-2}\left(s_{Q}-s_{p}\right) \Phi d s_{Q}\right| \leqq \frac{C_{n}^{\prime}}{\mu^{m+1}} C_{1, \gamma}^{\Phi}
$$

If, additionally, $\delta \leqq \frac{1}{4} \Lambda_{\gamma}$, then for $n \geqq 0$ and $j=0$,

$$
\left|\int_{\partial \Omega-N_{P}} \frac{d^{n}}{d R^{n}} K_{0}(\mu r) \Phi d s_{Q}\right| \leqq \frac{C_{n}^{\prime \prime} \mu^{n}}{(\mu|\partial \Omega|)^{6}}\|\Phi\|_{0,1, \partial \Omega} e^{-\mu r}
$$

Proof: Estimate (4.11) follows from the estimate for $\left(d^{n} / d z^{n}\right) K_{0}(z)$ and $L^{n} K_{0}(z)$ in Lemma 3. For the rest, first consider the special case where $\Omega$ is the upper half-plane in $\mathbb{R}^{2}, N_{P}=\partial \Omega$ is the $y$-axis in $\mathbb{R}^{2}, r$ is measured along the $x$-axis and $\Phi \equiv 1$.

Let

$$
u(r)=\frac{1}{2 \pi} \int_{-\infty}^{\infty} K_{0}(\mu R) d y
$$

Then, $u$ satisfies the equation $-\nabla^{2} u+\mu^{2} u=l$, where $l$ is the line potential of unit density along the $y$-axis; i.e.,

$$
\int_{-\infty}^{\infty} l d x=1
$$

The equation is solved easily by reducing to the one-dimensional case:

$$
u(r)=\frac{1}{2 \mu} e^{-\mu r} .
$$

Therefore,

$$
\frac{1}{2 \pi} \int_{-\infty}^{\infty} K_{0}(\mu R) d y_{Q}=\frac{1}{2 \mu} e^{-\mu r}
$$

Differentiation of this formula and integration by parts show that estimates (4.1) through (4.8) hold such that the error terms in the first five estimates are actually zero. To obtain (4.9), just note that $R=\sigma=\left|y_{Q}-y_{P}\right|$ and

$$
\mu^{m+1} \int_{-\infty}^{\infty} \mathscr{L}^{n} K_{0}(\mu R) \sigma^{2 n+m} d y_{Q}=\int_{-\infty}^{\infty} \frac{1}{\mu \sigma}\left(\frac{d}{d(\mu \sigma)}\right)^{n} K_{0}(\mu \sigma)(\mu \sigma)^{2 n+m} d\left(\mu y_{Q}\right)
$$

which does not depend on $\mu$ and the integrand, at worst, has only logarithmic pole at $y_{Q}=y_{P}$. The integrand in (4.10) has a pole of the order $1 /\left(y_{Q}-y_{P}\right)$ when $m=r=0$ and hence the integral is defined only as a limit as indicated. 
The numerator is an even function of $y_{Q}$ while the denominator is odd. Therefore, the integral is zero for all $r>0$.

Consider now the general case. The estimates in the lemma follow by performing a change of variables as follows. We let

$$
\eta=\left(s_{Q}-s_{P}\right) \sqrt{\frac{R^{2}-r^{2}}{\sigma^{2}}} .
$$

As before, we abbreviate $k_{\gamma}^{2} O\left(\sigma^{m}\right)$ and $k_{\gamma}^{2} O\left(\eta^{m}\right)$ as $O_{1}\left(\sigma^{m}\right)$ and $O_{1}\left(\eta^{m}\right)$, respectively. By Lemma 1 ,

$$
\frac{\eta}{s_{Q}-s_{P}} \frac{\partial \eta}{\partial s_{Q}}=\left(1-\kappa_{P} r\right)+r O_{1}(\sigma)+O_{1}\left(\sigma^{2}\right)
$$

and

$$
\frac{\eta}{s_{Q}-s_{P}}=\sqrt{\left(1-\kappa_{P} r\right)+r O_{1}(\sigma)+O_{1}\left(\sigma^{2}\right)}
$$

By Lemma $2, \frac{1}{4} \leqq \eta^{2} / \sigma^{2} \leqq 4$ and $\frac{1}{2} \leqq \eta /\left(s_{Q}-s_{P}\right) \leqq 2$. Hence, $\eta_{Q}$ is an invertible function of $s_{Q}$ :

$$
\begin{aligned}
\frac{d s_{Q}}{d \eta_{Q}} & =\frac{\sqrt{\left(1-\kappa_{P} r\right)+r O_{1}(\sigma)+O_{1}\left(\sigma^{2}\right)}}{\left(1-\kappa_{P} r\right)+r O_{1}(\sigma)+O_{1}\left(\sigma^{2}\right)} \\
& =\frac{1}{\sqrt{1-\kappa_{P} r}}+r O_{1}(\eta)+O_{1}\left(\eta^{2}\right) \\
& =1+r k_{\gamma} O(1)+k_{\gamma} O(\eta) \\
s_{Q}-s_{P} & =\frac{\eta}{\sqrt{1-\kappa_{P} r}}+r O_{1}\left(\eta^{2}\right)+O_{1}\left(\eta^{3}\right)=\eta+r k_{\gamma} O(\eta)+k_{\gamma} O\left(\eta^{2}\right)
\end{aligned}
$$

and

$$
\sigma^{2}=\frac{\eta^{2}}{\sqrt{1-\kappa_{P} r}}+r O_{1}\left(\eta^{3}\right)+O_{1}\left(\eta^{4}\right)=\eta^{2}+r k_{\gamma} o\left(\eta^{2}\right)+k_{\gamma} O\left(\eta^{3}\right)
$$

Note also that, if $|\eta| \geqq 2 \delta$,

$$
1 \leqq\left|\frac{R}{\eta}\right|=\sqrt{\frac{\eta^{2}+r^{2}}{\eta^{2}}} \leqq 2
$$

and

$$
1 \leqq\left|\frac{d \eta_{Q}}{d R_{Q}}\right|=\left|\frac{R}{\eta}\right| \leqq 2
$$


We also need three more integral estimates:

(i)

$$
\begin{aligned}
\int_{0}^{\infty} K_{0}(\mu R) \eta d \eta \\
=-\frac{1}{\mu} \int_{r}^{\infty} \frac{d}{d R}\left(R K_{1}(\mu R)\right) d R \\
=\frac{r K_{1}(\mu r)}{\mu} \leqq \max (1, \sqrt{\mu r}) e^{-\mu r} O\left(\frac{1}{\mu^{2}}\right)
\end{aligned}
$$

(ii)

$$
\begin{gathered}
\int_{0}^{\infty} \mathscr{L} K_{0}(\mu R) \eta d \eta \\
=-\int_{r}^{\infty} \frac{d K_{0}(\mu R)}{d R} d R \\
=K_{0}(\mu r) \leqq \frac{e^{-\mu r}}{\sqrt{\mu r}} O(1)
\end{gathered}
$$

Integration by parts gives us

$$
\text { (iii) } \int_{0}^{\infty} \mathscr{L} K_{0}(\mu R) \eta^{3} d \eta=\frac{2 r K_{1}(\mu r)}{\mu} \leqq \max (1, \sqrt{\mu r}) e^{-\mu r} O\left(\frac{1}{\mu^{2}}\right) \text {. }
$$

Finally, note that if $j=1$,

$$
\Phi=\Phi_{P}+C_{1, \gamma}^{\Phi} O(\eta)
$$

and if $j=2$,

$$
\Phi=\Phi_{P}+\left|\frac{d \Phi}{d s}\right|_{P} \eta+C_{2, \gamma}^{\Phi}\left[r O(\eta)+O\left(\eta^{2}\right)\right]
$$

Consider now the first estimate in the lemma with $j=2$. Perform the change of variables and substitute the above estimates for $d s_{Q} / d \eta_{Q}$ and $\Phi$. Let $\eta_{-}$and $\eta_{+}$be the limits of $\eta_{Q}$ corresponding to the limits of $s_{Q}$ in $N_{P}$. We get

$$
\begin{aligned}
& \frac{1}{2 \pi} \int_{N_{P}} K_{0}(\mu R) \Phi d s_{Q} \\
& \quad=\frac{1}{2 \pi} \int_{\eta_{-}}^{\eta_{+}} K_{0}(\mu R)\left\{\frac{\Phi_{P}}{\sqrt{1-\kappa_{P} r}}+\left.\frac{d \Phi}{d s}\right|_{P} \eta+C_{2, y}^{\Phi}\left[r O(\eta)+O\left(\eta^{2}\right)\right]\right\} d \eta_{Q} .
\end{aligned}
$$

Let $I$ be the interval $\left[\eta_{-}, \eta_{+}\right]$and $J=(-\infty, \infty)-\left(\eta_{-}, \eta_{+}\right)$,

$$
\frac{1}{2 \pi} \int_{I} K_{0}(\mu R) d \eta_{Q}=\frac{1}{2 \pi} \int_{-\infty}^{\infty} K_{0}(\mu R) d \eta_{Q}-\frac{1}{2 \pi} \int_{J} K_{0}(\mu R) d \eta_{Q}
$$


By the special case above,

$$
\frac{1}{2 \pi} \int_{-\infty}^{\infty} K_{0}(\mu R) d \eta_{Q}=\frac{e^{-\mu r}}{2 \mu}
$$

Since $\left|\eta_{ \pm}\right| \geqq 2 \delta$ by Lemma 2 ,

$$
\begin{aligned}
&\left|\int_{J} K_{0}(\mu R) d \eta_{Q}\right| \leqq \int_{2 \delta}^{\infty} K_{0}(\mu R) d \eta_{Q} \\
& \leqq 2 \int_{2 \delta}^{\infty} K_{0}(\mu R) \frac{d \eta_{Q}}{d R_{Q}} d R_{Q} \\
& \leqq 4 \int_{2 \delta}^{\infty} K_{0}(\mu R) d R \\
& \leqq e^{-2 \mu \delta} O\left(\frac{1}{\mu}\right) \\
& \leqq \frac{e^{-\mu r}}{\mu^{6}|\partial \Omega|^{6}} O\left(\frac{1}{\mu}\right) \\
& \leqq k_{\gamma}^{2} e^{-\mu r} O\left(\frac{1}{\mu^{3}}\right) \\
& \int_{I} K_{0}(\mu R) \eta d \eta_{Q}=\int_{-\infty}^{\infty} K_{0}(\mu R) \eta d \eta_{Q}-\int K_{0}(\mu R) \eta d \eta_{Q}
\end{aligned}
$$

The first integral is zero because $\eta$ is an odd function while $K_{0}(\mu R)$ is even. Thus

$$
\begin{aligned}
\left|\int_{J} K_{0}(\mu R) \eta d \eta_{Q}\right| & \leqq 2 \int_{2 \delta}^{\infty} K_{0}(\mu R) \eta d \eta_{Q} \\
& \leqq 2 \int_{2 \delta}^{\infty} K_{0}(\mu R) R d R \\
& \leqq(\mu \delta)^{1 / 2} e^{-2 \mu \delta} O\left(\frac{1}{\mu^{2}}\right) \\
& \leqq k_{\gamma} e^{-\mu r} O\left(\frac{1}{\mu^{3}}\right) \\
\int_{I} K_{0}(\mu R)|\eta| d \eta & \leqq 2 \int_{0}^{\infty} K_{0}(\mu R) \eta d \eta \leqq \max (1, \sqrt{\mu r}) e^{-\mu r} O\left(\frac{1}{\mu^{2}}\right) \\
\int_{I} K_{0}(\mu R) \eta^{2} d \eta & \leqq \int_{-\infty}^{\infty} K_{0}(\mu R) \eta^{2} d \eta_{Q} \\
& \leqq(1+\mu r) e^{-\mu r} O\left(\frac{1}{\mu^{3}}\right), \quad \text { by the special case. }
\end{aligned}
$$


Putting all of the above estimates together, we get the first estimate in the lemma. The other estimates are obtained in the same way.

Proof of the Theorem (Part B): For $1 \leqq k \leqq 4$, define

$$
\gamma_{k}=\left\{P \in \gamma^{*} \mid \operatorname{arc} \operatorname{distance}(P, \gamma) \leqq 4 k \delta\right\} \subset \gamma^{*} \text {. }
$$

For a point $P \in \gamma_{3}$, define its $4 \delta$-neighborhood $N_{P}$ in $\partial \Omega$ as in Lemma 4. We keep $N_{P}$ fixed when we differentiate with respect to $s_{P}$.

We begin with the Green's formula:

$$
v(P)=-\frac{1}{\pi} \int_{\partial \Omega_{Q}} K_{0}(\mu R) \frac{\partial v}{\partial n_{Q}} d s_{Q}+\frac{1}{\pi} \int_{\partial \Omega_{Q}} \frac{\partial K_{0}(\mu R)}{\partial n_{Q}} v(Q) d s_{Q} .
$$

First, consider these integrals over $\partial \Omega-N_{P}$, where $P$ is a point in $\gamma_{3} . R^{2}$ and $R \partial R / \partial n_{Q}$ are $C^{2,1}$-functions of $s_{P}$. Moreover, by Lemma $2, R \geqq 2 \delta$ over $\partial \Omega-N_{p}$. Therefore, $R$ and $\partial R / \partial n_{Q}$ are $C^{2,1}$-functions of $s_{P} . K_{0}(\mu R)$ is an analytic function of $R$ if $R \neq 0$. Therefore, $K_{0}(\mu R)$ and $\partial K_{0}(\mu R) / \partial n_{Q}$ are $C^{2,1}$-functions of $s_{P}$ over $\partial \Omega-N_{P}$. We may compute their derivatives by differentiating inside the integral sign. Let $\psi$ denote $\partial v / \partial n$. Let

$$
w_{1}=-\frac{1}{\pi} \int_{\partial \Omega-N_{P}} K_{0}(\mu R) \psi d s_{Q} .
$$

By estimate (4.11) in Lemma 4,

$$
\begin{gathered}
\left|w_{1}\right|=\frac{\|\psi\|_{0,1, \partial \Omega}}{\mu^{3}|\partial \Omega|^{6}} O\left(\frac{1}{\mu^{3}}\right) \\
\frac{d w_{1}}{d s}=-\frac{1}{\pi} \int_{\partial \Omega-N_{P}} \frac{d}{d R} K_{0}(\mu R) \frac{\partial R}{\partial s_{P}} \psi d s_{Q}
\end{gathered}
$$

where $\left|\partial R / \partial s_{P}\right| \leqq 1$ by Lemma 1 . Therefore,

$$
\left|\frac{d w_{1}}{d s_{P}}\right|=\frac{\|\psi\|_{0,1, \partial \Omega}}{\mu^{3}|\partial \Omega|^{6}} O\left(\frac{1}{\mu^{2}}\right) \text {. }
$$

Let

$$
\begin{gathered}
w_{2}=\frac{1}{\pi} \int_{\partial \Omega-N_{p}} \frac{\partial K_{0}(\mu R)}{\partial \eta_{Q}} v(Q) d s_{Q} \\
\frac{\partial K_{0}(\mu R)}{\partial \eta_{Q}}=\frac{d}{d R} K_{0}(\mu R) \frac{\partial R}{\partial n_{Q}} \text { and }\left|\frac{\partial R}{\partial n_{Q}}\right| \leqq 1
\end{gathered}
$$

Consequently,

$$
\begin{gathered}
\left|w_{2}\right|=\frac{\|v\|_{0,1, \partial \Omega}}{\mu^{3}|\partial \Omega|^{6}} o\left(\frac{1}{\mu^{2}}\right)=\frac{\|v\|_{0, \infty, \partial \Omega}}{\mu|\partial \Omega|^{4}} O\left(\frac{1}{\mu^{3}}\right) \\
\frac{d w_{2}}{d s}=\frac{1}{\pi} \int_{\partial \Omega-N_{P}}\left[\frac{d^{2}}{d R^{2}} K_{0}(\mu R) \frac{\partial R}{\partial s_{P}} \frac{\partial R}{\partial n_{Q}}+\frac{d}{d R} K_{0}(\mu R) \frac{\partial}{\partial s_{P}} \frac{\partial R}{\partial n_{Q}}\right] v(Q) d s_{Q}
\end{gathered}
$$


By Lemma 2,

$$
\left|\frac{\partial R}{\partial s_{P}} \frac{\partial R}{\partial n_{Q}}\right| \leqq 1 \text { and }\left|\frac{\partial}{\partial s_{P}} \frac{\partial R}{\partial n_{Q}}\right| \leqq \frac{1}{R} \leqq \frac{1}{2} \mu
$$

Therefore,

$$
\left|\frac{d w_{2}}{d s}\right|=\frac{\|v\|_{0, \infty, \partial \Omega}}{\mu|\partial \Omega|^{4}} O\left(\frac{1}{\mu^{2}}\right)
$$

Consider now the same integrals over $N_{p}$. Since $K_{0}(\mu R)$ has a logarithmic pole, differentiation inside the integral sign produces divergent integrals in most cases. Hence we proceed as follows. Let $T_{P, \delta}=[0, \delta] \times N_{P}$. If an integral has the form

$$
\int_{N_{P}} G(P, Q) \Phi(Q) d s_{Q}
$$

where $G$ is a function on $T_{P, \delta} \times N_{P}$ and $\Phi$ is a function on $N_{P}$, then

$$
\begin{aligned}
& \frac{d}{d s} \int_{N_{P}} G(P, Q) \Phi(Q) d s_{Q}= \int_{N_{P}} \frac{\partial}{\partial s_{P}} G(P, Q) \Phi(Q) d s_{Q} \\
&= \int_{N_{P}}\left[-\frac{\partial}{\partial s_{Q}}+\partial_{\sigma}\right] G(P, Q) \Phi(Q) d s_{Q} \\
& \text { where } \partial_{\sigma}=\partial / \partial s_{P}+\partial / \partial s_{Q} \\
&=-[G(P, Q) \Phi(Q)]_{Q_{-}}^{Q_{+}}+\int_{N_{P}} G(P, Q) \frac{d \Phi}{d s_{Q}} d s_{Q} \\
& \\
&+\int_{N_{P} \partial_{\sigma} G(P, Q) \Phi(Q) d s_{Q}}
\end{aligned}
$$

where $Q_{ \pm}$are the end points of the arc $N_{P} . \partial_{\sigma} G(P, Q)$ will turn out to have singularity no worse than the singularity of $G(P, Q)$. Therefore, everything in the last step is convergent if the original integral is. If any of the integrals in any of the steps above turn out to be divergent we consider the integral

$$
\int_{N_{P}} G\left(P^{\#}, Q\right) \Phi(Q) d s_{Q}
$$

where $P^{\#}$ is a point in $T_{P, \delta}$ with coordinates $\left(r, s_{P}\right)$, carry out the above steps and then take the limit as $r \rightarrow 0$. 
Let $v_{1}(P)=-(1 / \pi) \int_{N_{P}} K_{0}(\mu R) \psi(Q) d s_{Q}$. By (4.1) in Lemma 4,

$$
\begin{aligned}
v_{1}(P) & =\|\psi\|_{0, \infty, N_{P}} O\left(\frac{1}{\mu}\right) \\
& =-\frac{\psi_{P}}{\mu}+C_{1, \gamma^{*}}^{\psi} O\left(\frac{1}{\mu^{2}}\right) \\
& =-\frac{\psi_{P}}{\mu}+C_{2, \gamma^{*}}^{\psi} O\left(\frac{1}{\mu^{3}}\right) .
\end{aligned}
$$

Consider $v_{1}\left(P^{\#}\right)=-(1 / \pi) \int_{N_{P}} K_{0}\left(\mu R^{\#}\right) \psi(Q) d s_{Q}$ where $R^{\#}=\left|P^{\#} Q\right|$. Then,

$$
\begin{aligned}
\frac{\partial v_{1}}{\partial s}= & \frac{1}{\pi}\left[K_{0}\left(\mu R^{\#}\right) \psi(Q)\right]_{Q_{-}}^{Q_{+}}-\frac{1}{\pi} \int_{N_{P}} K_{0}\left(\mu R^{\#}\right) \frac{d \psi}{d s_{Q}} d s_{Q} \\
& +\frac{1}{2 \pi} \int \mathscr{L} K_{0}\left(\mu R^{\#}\right) \partial_{\sigma}\left(R^{\#}\right)^{2} \psi(Q) d s_{Q},
\end{aligned}
$$

where $\mathscr{L}=-(1 / R) d / d R$.

By Lemma 1,

$$
\partial_{\sigma}\left(R^{\#}\right)^{2}=\left[r O\left(\sigma^{2}\right)+O\left(\sigma^{3}\right)\right] k_{\gamma^{*}}^{2}
$$

Since $\mathscr{L} K_{0}(\mu R)$ has singularity like $1 / \sigma^{2}$ at $R=0$, the last integral is convergent when $r=0$. Therefore,

$$
\begin{aligned}
\frac{d v_{1}(P)}{d s}= & \frac{1}{\pi}\left[K_{0}(\mu R) \psi(Q)\right]_{Q}^{Q_{+}}+\frac{1}{2 \pi} \int_{N_{P}} \mathscr{L} K_{0}(\mu R) \partial_{\sigma} R^{2} \psi(Q) d s_{Q} \\
& -\frac{1}{\pi} \int_{N_{P}} K_{0}(\mu R) \frac{d \psi}{d s_{Q}} d s_{Q} .
\end{aligned}
$$

By Lemma 3, the first term equals

$$
\|\psi\|_{0, \infty, N_{p}} \frac{e^{-2 \mu \delta}}{\sqrt{2 \mu \delta}} O(1)=\frac{\|\psi\|_{0, \infty, \gamma^{*}}}{\mu^{4}|\partial \Omega|^{6}} O\left(\frac{1}{\mu^{2}}\right)=\frac{\|\psi\|_{0, \infty, \gamma^{*}}}{|\partial \Omega|^{2}} O\left(\frac{1}{\mu^{2}}\right) .
$$

Consider now the second term. Since $\partial_{\sigma} R^{2}=k_{\gamma^{*}}^{2} O\left(\sigma^{3}\right)$, the term equals $k_{\gamma^{*}}^{2}\|\psi\|_{0, \infty, N_{P}} O\left(1 / \mu^{2}\right)$ by (4.9) in Lemma 4. By (4.1) again,

$$
-\frac{1}{\pi} \int_{N_{P}} K_{0}(\mu R) \frac{d \psi}{d s_{Q}} d s_{Q}=\left\|\psi^{\prime}\right\|_{0, \infty, N_{P}} O\left(\frac{1}{\mu}\right)=-\frac{1}{\mu} \psi_{P}^{\prime}+C_{1, \gamma^{*}}^{\psi^{\prime}} O\left(\frac{1}{\mu^{2}}\right) .
$$


680

D. MUMFORD AND J. SHAH

Putting all the pieces together, we get

$$
\frac{d v_{1}}{d s}=C_{1, \gamma^{*}}^{\psi} O\left(\frac{1}{\mu}\right)=-\frac{1}{\mu} \psi_{P}^{\prime}+C_{2, \gamma^{*}}^{\psi} O\left(\frac{1}{\mu^{2}}\right) .
$$

Consider

$$
v_{2}=\frac{1}{\mu} \int_{N_{P}} \frac{\partial K_{0}(\mu R)}{\partial n_{Q}} v(Q) d s_{Q}=-\frac{1}{\pi} \int_{N_{P}} \mathscr{L} K_{0}(\mu R) R \frac{\partial R}{\partial n_{Q}} v(Q) d s_{Q} .
$$

Let

$$
\|\| v\left\|_{0, \gamma^{*}}=\right\| \frac{\partial v}{\partial n}\left\|_{0, \infty, \gamma^{*}}+\frac{k_{\gamma^{*}}^{2}}{\mu}\right\| v \|_{0, \infty, \partial \Omega}+\frac{\|\partial v / \partial n\|_{0,1, \partial \Omega}}{\mu^{5}|\partial \Omega|^{6}}
$$

Since $v$ appears on both sides of the equation, we have to bootstrap the result, successively shrinking the neighborhood of $\gamma$, from $\gamma_{4}$ to $\gamma_{1}$, during the process.

Start with $P \in \gamma_{3}$. Since $R \partial R / \partial n_{Q}=k_{\gamma^{*}} O\left(\sigma^{2}\right)$,

$$
v_{2}(P)=\|v\|_{0, \infty, \gamma^{*}} k_{\gamma^{*}} O\left(\frac{1}{\mu}\right) .
$$

Hence, for all $P \in \gamma_{3}$,

$$
\begin{aligned}
v(P) & =w_{1}(P)+w_{2}(P)+v_{1}(P)+v_{2}(P) \\
& =\left[\|\psi\|_{0, \infty, \gamma^{*}}+k_{\gamma^{*}}\|v\|_{0, \infty, \partial \Omega}+\frac{\|\psi\|_{0,1, \partial \Omega}}{\mu^{5}|\partial \Omega|^{6}}\right] O\left(\frac{1}{\mu}\right) .
\end{aligned}
$$

Restrict $P$ now to $\gamma_{2}$ so that $N_{P} \subset \gamma_{3}$. Then,

$$
\begin{aligned}
v_{2}(P) & =\|v\|_{0, \infty, N_{P}} K_{\gamma^{*}} O\left(\frac{1}{\mu}\right)=\|v\|_{0, \gamma^{*}} O\left(\frac{1}{\mu}\right), \\
v(P) & =\|v\|_{0, \gamma^{*}} O\left(\frac{1}{\mu}\right)
\end{aligned}
$$

Next, restrict $P$ to $\gamma_{1}$ so that $N_{P} \subset \gamma_{2}$. Then,

$$
v_{2}(P)=k_{\gamma^{*}}\|v\|_{0, \gamma^{*}} o\left(\frac{1}{\mu^{2}}\right),
$$

and

$$
v(P)=-\frac{\psi(P)}{\mu}+\|\| v \|_{1, \gamma^{*}} o\left(\frac{1}{\mu^{2}}\right)
$$


which essentially proves Part A of the theorem. Finally, restrict $P$ to $\gamma$. Let $u=v+\psi / \mu$. Then,

$$
\begin{aligned}
v_{2}(P)= & -\frac{1}{\pi} \int_{N_{P}} \mathscr{L} K_{0}(\mu R) R \frac{\partial R}{\partial n_{Q}} v(Q) d s_{Q} \\
= & -\frac{1}{\pi} \int_{N_{P}} \mathscr{L} K_{0}(\mu R)\left[-\frac{1}{2} \kappa_{P} \sigma^{2}+k_{\gamma^{*}}^{2} O\left(\sigma^{3}\right)\right]\left[-\frac{\psi(Q)}{\mu}+u(Q)\right] d s_{Q} \\
= & -\frac{\kappa_{P}}{2 \pi \mu} \int_{N_{P}} \mathscr{L} K_{0}(\mu R) \sigma^{2} \psi(Q) d s_{Q} \\
& +\frac{1}{\mu} \int_{N_{P}} \mathscr{L} K_{0}(\mu R) k_{\gamma^{*}}^{2} O\left(\sigma^{3}\right) \psi(Q) d s_{Q} \\
& +\int_{N_{p}} \mathscr{L} K_{0}(\mu R) k_{\gamma^{*}} O\left(\sigma^{2}\right) u(Q) d s_{Q} .
\end{aligned}
$$

By (4.3),

$$
\begin{aligned}
& \text { the first integral }=-\frac{\kappa_{P}}{2 \mu^{2}} \psi(P)+k_{\gamma}^{*} C_{1, \gamma^{*}}^{\psi} O\left(\frac{1}{\mu^{3}}\right), \\
& \text { the second integral }=k_{\gamma^{*}}^{2}\|\psi\|_{0, \infty, \gamma^{*}} O\left(\frac{1}{\mu^{3}}\right),
\end{aligned}
$$

and

$$
\text { the third integral }=k_{\gamma^{*}}\|u\|_{0, \infty, N_{P}} O\left(\frac{1}{\mu}\right)=k_{\gamma^{*}}\|v\|_{1, \gamma^{*}} O\left(\frac{1}{\mu^{3}}\right)
$$

Therefore,

$$
v_{2}(P)=-\frac{\kappa_{P}}{2 \mu^{2}} \psi_{P}+\|v\|_{2, \gamma^{*}} O\left(\frac{1}{\mu^{3}}\right)
$$

and

$$
v(P)=-\left(1+\frac{\kappa_{P}}{2 \mu}\right) \frac{\psi_{P}}{\mu}+\|v\|_{2, \gamma^{*}} O\left(\frac{1}{\mu^{3}}\right)
$$


It remains to estimate $d v_{2} / d s$. For all $P \in \gamma$,

$$
\begin{aligned}
\frac{d v_{2}}{d s}=-\frac{1}{\pi} \frac{d}{d s} \int_{N_{P}} \mathscr{L} K_{0}(\mu R) R \frac{\partial R}{\partial n_{Q}}\left(-\frac{\psi(Q)}{\mu}+u(Q)\right) d s_{Q} \\
\frac{1}{\pi \mu} \frac{d}{d s} \int_{N_{P}} \mathscr{L} K_{0}(\mu R) R \frac{\partial R}{\partial n_{Q}} \psi(Q) d s_{Q} \\
=-\frac{1}{\pi \mu}\left[\mathscr{L} K_{0}(\mu R) R \frac{\partial R}{\partial n_{Q}} \psi(Q)\right]_{Q_{-}}^{Q_{+}} \\
+\frac{1}{\pi \mu} \int_{N_{P}} \partial_{\sigma}\left[\mathscr{L} K_{0}(\mu R) R \frac{\partial R}{\partial n_{Q}}\right] \psi(Q) d s_{Q} \\
+\frac{1}{\pi \mu} \int_{N_{p}} \mathscr{L} K_{0}(\mu R) R \frac{\partial R}{\partial n_{Q}} \frac{d \psi}{d s_{Q}} d s_{Q}
\end{aligned}
$$

The first term is easily seen to be of order $k_{\gamma^{*}}^{2}\|\psi\|_{0, \infty, \gamma^{*}} O\left(1 / \mu^{2}\right)$ :

$$
\begin{aligned}
& \frac{1}{\pi \mu} \int_{N_{P}:} \partial_{\sigma}\left[\mathscr{L} K_{0}(\mu R) R \frac{\partial R}{\partial n_{Q}}\right] \psi(Q) d s_{Q} \\
& =\frac{1}{\pi \mu} \int_{N_{p}:}\left[-\frac{1}{2} \mathscr{L} R^{2} \mathscr{L} K_{0}(\mu R)\left(\partial_{\sigma} R^{2}\right)\left(\frac{1}{R} \frac{\partial R}{\partial n_{Q}}\right)\right. \\
& \left.+\mathscr{L} K_{0}(\mu R) R^{2} \partial_{\sigma}\left(\frac{1}{R} \frac{\partial R}{\partial n_{Q}}\right)\right] \psi(Q) d s_{Q} \\
& =-\frac{1}{\pi \mu} \int_{N_{P}}\left[\frac{1}{2} \mu^{2} K_{0}(\mu R)+\mathscr{L} K_{0}(\mu R)\right]\left(\partial_{\sigma} R^{2}\right)\left(\frac{1}{R} \frac{\partial R}{\partial n_{Q}}\right) \psi(Q) d s_{Q} \\
& +\frac{1}{\pi \mu} \int_{N_{P}} \mathscr{L} K_{0}(\mu R) \partial_{\sigma}\left(R \frac{\partial R}{\partial n_{Q}}\right) \psi(Q) d s_{Q} .
\end{aligned}
$$

By Lemmas 1 and 2,

$$
\left(\frac{1}{R} \frac{\partial R}{\partial n_{Q}}\right)\left(\partial_{\sigma} R^{2}\right)=\frac{k_{\gamma^{*}}^{2} O\left(\sigma^{2}\right) O\left(\sigma^{2}\right)}{R^{2}}=k_{\gamma^{*}}^{2} O\left(\sigma^{2}\right)
$$

and

$$
\partial_{\sigma}\left(R \frac{\partial R}{\partial n_{Q}}\right)=k_{\gamma^{*}}^{2} O\left(\sigma^{2}\right)
$$


Therefore, by (4.3) and (4.6),

$$
\frac{1}{\pi \mu} \int_{N_{P}} \partial_{\sigma}\left[\mathscr{L} K_{0}(\mu R) R \frac{\partial R}{\partial n_{Q}}\right] \psi(Q) d s_{Q}=k_{\gamma^{*}}^{2}\|\psi\|_{0, \infty, \gamma^{*}} O\left(\frac{1}{\mu^{2}}\right)
$$

Since $R \partial R / \partial n_{Q}=k_{\gamma^{*}} O\left(\sigma^{2}\right)$,

$$
\frac{1}{\pi \mu} \int_{N_{p}} \mathscr{L} K_{0}(\mu R) R \frac{\partial R}{\partial n_{Q}} \frac{d \psi}{d s_{Q}} d s_{Q}=k_{\gamma^{*}}\left\|\psi^{\prime}\right\|_{0, \infty, \gamma^{*}} O\left(\frac{1}{\mu^{2}}\right)
$$

The remaining integral is

$$
\begin{aligned}
& =-\frac{1}{\pi} \frac{d}{d s} \int_{N_{P}} \mathscr{L} K_{0}(\mu R) R \frac{\partial R}{\partial n_{Q}} u(Q) d s_{Q} \\
& =-\frac{1}{\pi} \int_{N_{P}}\left[\mathscr{L} R^{2} \mathscr{L} K_{0}(\mu R)\left(R \frac{\partial R}{\partial s_{P}}\right)\left(\frac{1}{R} \frac{\partial R}{\partial n_{Q}}\right)\right. \\
& \left.\quad-R^{2} \mathscr{L} K_{0}(\mu R) \frac{\partial}{\partial s_{P}}\left(\frac{1}{R} \frac{\partial R}{\partial n_{Q}}\right)\right] u(Q) d s_{Q} \\
& =\frac{\mu^{2}}{\pi} \int_{N_{P}} K_{0}(\mu R)\left(R \frac{\partial R}{\partial s_{P}}\right)\left(\frac{1}{R} \frac{\partial R}{\partial n_{Q}}\right) u(Q) d s_{Q} \\
& -\frac{1}{\pi} \int_{N_{P}} \mathscr{L} K_{0}(\mu R)\left[\frac{\partial}{\partial s_{P}}\left(R \frac{\partial R}{\partial n_{Q}}\right)-2\left(R \frac{\partial R}{\partial s_{P}}\right)\left(\frac{1}{R} \frac{\partial R}{\partial n_{Q}}\right)\right] u(Q) d s_{Q} .
\end{aligned}
$$

Since

$$
\left(R \frac{\partial R}{\partial s_{P}}\right)\left(\frac{1}{R} \frac{\partial R}{\partial n_{Q}}\right)=\frac{O(\sigma) k_{\gamma^{*}} O\left(\sigma^{2}\right)}{R^{2}}=k_{\gamma^{*}} O(\sigma)
$$

the first integral $=\mu^{2}\|u\|_{0, \infty, N_{p}} k_{\gamma^{*}} O\left(\frac{1}{\mu^{2}}\right)=k_{\gamma^{*}}\|v\| \|_{1, \gamma^{*}} O\left(\frac{1}{\mu^{2}}\right)$ 
In the second integral,

$$
\begin{aligned}
\frac{1}{R^{2}}\left[R^{2} \frac{\partial}{\partial s_{P}}\left(R \frac{\partial R}{\partial n_{Q}}\right)-2\left(R \frac{\partial R}{\partial s_{P}}\right)\left(R \frac{\partial R}{\partial n_{Q}}\right)\right] \\
=\frac{1}{R^{2}}\left\{\left[\sigma^{2}+k_{\gamma^{*}} O\left(\sigma^{4}\right)\right]\left[\kappa_{P}\left(s_{Q}-s_{P}\right)+\kappa_{\gamma^{*}}^{2} O\left(\sigma^{2}\right)\right]\right. \\
\left.\quad-2\left[\left(s_{Q}-s_{P}\right)+k_{\gamma^{*}}^{2} O\left(\sigma^{3}\right)\right]\left[\frac{1}{2} \kappa_{P} \sigma^{2}+k_{\gamma^{*}}^{2} O\left(\sigma^{3}\right)\right]\right\} \\
=k_{\gamma^{*}}^{2} O\left(\sigma^{2}\right) .
\end{aligned}
$$

Therefore,

$$
\begin{aligned}
\text { the second integral } & =k_{\gamma^{*}}^{2}\|u\|_{0, \infty, N_{p}} O\left(\frac{1}{\mu}\right) \\
& =k_{\gamma^{*}}^{2}\|v\|_{1, \gamma^{*}} O\left(\frac{1}{\mu^{3}}\right) \\
& =k_{\gamma^{*}}\|v\|_{1, \gamma^{*}} O\left(\frac{1}{\mu^{2}}\right)
\end{aligned}
$$

Adding up all the estimates, we get

$$
\frac{d v_{2}}{d s}(P)=\|\| v \|_{2, \gamma^{*}} O\left(\frac{1}{\mu^{2}}\right)
$$

and

$$
\frac{d v}{d s}(P)=-\frac{\psi_{P}^{\prime}}{\mu}+\|\| v \|_{2, \gamma^{*}} O\left(\frac{1}{\mu^{2}}\right)
$$

Acknowledgment. The first author gratefully acknowledges the Army Research Office and the National Science Foundation for support under grants DAAL03-86-K-0171 and IST-85-12736. The second author gratefully acknowledges the support of the National Science Foundation under grant IRI-8704467.

\section{Bibliography}

[1] Allard, W. K., and Almgren, F. J., The structure of stationary one-dimensional varifolds with positive density, Inventiones Math. 34, 1976, pp. 83-97.

[2] Abramowitz, M., and Stegun, I. A., eds., Handbook of Mathematical Functions, National Bureau of Standards, Applied Mathematics Series, 1965.

[3] Buser, P., On Cheeger's inequality, Proc. of Symp. in Pure Math. 36, 1980. 
[4] Blake, A., and Zisserman, A., Using Weak Continuity Constraints, Report CSR-186-85, Dept. of Comp. Sci., Edinburgh University, 1985.

[5] Freidlin, M., Functional Integration and Partial Differential Equations, Annals of Math. Studies, Princeton University Press, 1985.

[6] Geman, S., and Geman, D., Stochastic relaxation, Gibbs distributions, and the Bayesian restoration of images, IEEE Trans., PAMI 6, 1984, pp. 721-741.

[7] Grisvard, P., Elliptic Problems in Nonsmooth Domains, Pitman, 1985.

[8] Gurtin, M. E., On a theory of phase transitions with interfacial energy, Archive Rat. Mech. Anal. 87, 1985, pp. 187-212.

[9] Kondratiev, V. A., Boundary value problems for elliptic equations in domains with conical or angular points, Trans. Moscow Math. Soc. 16, 1967, pp. 227-313.

[10] Marroquin, J. L., Surface reconstruction preserving discontinuities, Artificial Intelligence Lab. Memo 792, M.I.T, 1984.

[11] McKean, H., Stochastic Integrals, Academic Press, 1969.

[12] Rice, J. R., Mathematical analysis in the mechanics of fracture, in Fracture, An Advanced Treatise, H. Liebowitz, ed., Academic Press, 1968.

[13] Simon, L., Lectures on Geometric Meáasure Theory, Proc. Centre for Math. Anal., Australian Nat. Univ., 3, 1983.

[14] Whittaker, E. T., and Watson, G. N., A Course of Modern Analysis, 4th ed., Cambridge University Press, 1952.

Received July, 1988. 